\title{
A Stefan problem for a protocell model with symmetry-breaking bifurcations of analytic solutions
}

\author{
AVNER FRIEDMAN \\ Department of Mathematics, University of Minnesota, Minneapolis, MN 55455, USA \\ BEI HU \\ Department of Mathematics, University of Notre Dame, Notre Dame, IN 46556, USA
}

AND

JUAN J. L. VELAZQUEZ

Departamento de Matematica Aplicada, Facultad de Matematicas, Universidad Complutense, 28040 Madrid, Spain

[Received 15 December 1999 and in revised form 12 July 2000]

\begin{abstract}
A simple model of a living cell which undergoes processes of growth and dissolution is described as a free boundary problem for a system of two reaction-diffusion equations; the condition on the free boundary is of the Stefan type. The special case of radially symmetric cells was studied in earlier work. This paper is concerned with the existence of symmetry-breaking stationary solutions, i.e. with solutions which are not radially symmetric. It is proved, in the two-dimensional case, that there exist branches of non-radial stationary solutions bifurcating from radially symmetric solutions; indeed, for any mode $l, l \geqslant 2$, there exists a unique bifurcation branch whose free boundary has the form $r=R_{l}+\varepsilon \cos l \theta+\sum_{n \geqslant 2} \varepsilon^{n} \lambda_{n}(\theta),|\varepsilon|$ small, with $\lambda_{n}(\theta)$ orthogonal to $\cos l \theta$.
\end{abstract}

Keywords: Stefan problem; protocell; bifurcation; symmetry-breaking

\section{The model}

We denote a variable point in $\mathbb{R}^{2}$ by $x=\left(x_{1}, x_{2}\right)$ or, in polar coordinates, by $(r, \theta)$. Consider the following free boundary problem: find a two-dimensional bounded domain $\Omega$ and functions $\mu$ and $u$ defined, respectively, in $\mathbb{R}^{2}$ and $\Omega$, such that

$$
\begin{aligned}
& \Delta \mu=\chi_{\Omega} \mu \quad \text { in } \mathbb{R}^{2}, \\
& \mu=\log r(1+\mathrm{o}(1)) \quad \text { as } r=|x| \rightarrow \infty, \\
& \mu \text { is continuously differentiable across } \partial \Omega, \\
& -\Delta u=\mu \quad \text { in } \Omega, \\
& u=0 \quad \text { on } \partial \Omega, \\
& \frac{\partial u}{\partial n}+\beta=0 \quad \text { on } \partial \Omega \quad(\beta>0) .
\end{aligned}
$$

Here $\chi_{\Omega}$ denotes the characteristic function of $\Omega$; we shall sometimes use the notation

$$
\mu^{+}=\left.\mu\right|_{\mathbb{R}^{2} \backslash \Omega}, \quad \mu^{-}=\left.\mu\right|_{\bar{\Omega}} .
$$


The above system is a two-dimensional version of a stationary 'protocell'; the concept of a protocell was introduced in [8] and [9] and it attempts to capture some of the average physical and chemical properties of growth and dissolution of a living cell. The function $\mu$ represents the nutrient concentration in the entire space with a given source at $\infty$, and the function $u$ is defined as $C-C^{*}$, where $C$ is the concentration of the fluid-like building material of the cell, and $C^{*}$ is the equilibrium concentration of the building material, so that $u$ vanishes at the cell's boundary $\partial \Omega$. The cell feeds on the nutrients in accordance with (1.4). Dissolution of the cell, at the rate $\beta$ across the boundary, represents waste removal through the cell. Actually, the model studied in [8] and [9] is somewhat different, and equally motivated, but the present version is mathematically a little simpler.

It is easy to check that for any $\beta>0$ there exists a unique radial solution

$$
\mu=\mu(r), \quad u=u(r), \quad \Omega=\{r<R\}
$$

and, in fact, $R=1 / \beta$. The purpose of this paper is to prove that there exist also non-radial solutions. We shall prove that for any integer $l \geqslant 2$ there exists a unique radius $r=R_{0 l}$ which is a bifurcation point of a family of analytic symmetry-breaking solutions with free boundary

$$
r=R_{0 l}+\varepsilon \cos l \theta+\sum_{n=2}^{\infty} \varepsilon^{n} \lambda_{\ln }(\theta)
$$

and

$$
\beta=\beta_{l 0}+\sum_{n=2}^{\infty} \varepsilon^{n} \beta_{\ln } \quad\left(\beta_{l 0}=\frac{1}{R_{0 l}}\right) .
$$

The fact that $\beta$ depends on $\varepsilon$ is a common feature of all bifurcation problems: one parameter of the system (in our case, $\beta$ ) is always dependent on the $\varepsilon$-parameter of the bifurcating family of solutions.

The corresponding solution $u_{l}, \mu_{l}^{+}, \mu_{l}^{-}$of (1.1)-(1.6) are also analytic in $(r, \theta, \varepsilon)$ in their respect regions, up to the boundary. Each bifurcation branch is uniquely determined under the following assumptions:

$$
\begin{aligned}
& u \text { and } \mu \text { are even functions in } \theta, \\
& \int_{0}^{2 \pi} u(r, \theta, \varepsilon) \cos \theta \mathrm{d} \theta \equiv 0, \quad \int_{0}^{2 \pi} \mu(r, \theta, \varepsilon) \cos \theta \mathrm{d} \theta \equiv 0, \\
& \int_{0}^{2 \pi} \lambda_{\ln }(\theta) \cos m \theta \mathrm{d} \theta=0 \quad \text { for } n \geqslant 2 \quad \text { and } \quad m=1, l .
\end{aligned}
$$

Note that the orthogonality of $\lambda_{\ln }(\theta)$ to $\cos l \theta$ for $n \geqslant 2$ is achieved by a choice of the bifurcation parameter. If this condition is not satisfied for the expansions $(1.7),(1.8)$, then by setting

$$
\varepsilon^{\prime}=\varepsilon+\sum_{n=2}^{\infty} \varepsilon^{n} \lambda_{\ln }^{0} \quad \text { where } \quad \lambda_{\ln }^{0}=\int_{0}^{2 \pi} \lambda_{\ln }(\theta) \cos \theta \mathrm{d} \theta,
$$

we obtain a new family of solutions with

$$
r=R_{0}+\varepsilon^{\prime} \cos l \theta+\sum_{n=2}^{\infty}\left(\varepsilon^{\prime}\right)^{n} \widehat{\lambda}_{\ln }(\theta), \quad \beta=\beta_{l 0}+\sum_{n=2}^{\infty}\left(\varepsilon^{\prime}\right)^{n} \widehat{\beta}_{\ln }
$$


and $\widehat{\mu}\left(r, \theta, \varepsilon^{\prime}\right)=\mu(r, \theta, \varepsilon), \widehat{u}\left(r, \theta, \varepsilon^{\prime}\right)=u(r, \theta, \varepsilon)$ for which all the conditions in (1.9)-(1.11) are satisfied.

The general theory of bifurcation (see, for instance, [6] and [7]) deals with problems of the form

$$
F(\lambda, u)=0
$$

where $\lambda$ is a real parameter (like the parameter $\beta$ in our case) and $u$ varies in a fixed Banach space $X$. Suppose for simplicity that $F(\lambda, 0) \equiv 0$ for all $\lambda \in \mathbb{R}$. A bifurcation point $\left(\lambda_{0}, 0\right)$ is a point for which there exists a one-parameter family of nontrivial solutions $\lambda=\lambda(\varepsilon), v=u(\varepsilon)$ of (1.12) with $\lambda(0)=\lambda_{0}, u(0)=0$. The Liapunov-Schmidt procedure reduces the construction of a bifurcation branch from the infinite-dimensional Banach space $X$ to a finite-dimensional space:

Suppose $F(\lambda, u)$ maps $u \in X$ into a Banach space $Y$ such that $X \subset Y$, and $F$ is smooth. Set

$$
L_{0}=\frac{\partial F\left(\lambda_{0}, 0\right)}{\partial u}
$$

and assume that $L_{0}$ is a Fredholm operator with index zero. Denote its null space by $\mathcal{N}$ (so that $n=\operatorname{dim} \mathcal{N}<\infty$ ) and its range by $\mathcal{R}_{0}$. Denote by $P$ the projection of $Y$ into $\mathcal{N}$ and by $Q$ the projection of $Y$ into $\mathcal{R}_{0}$. If we decompose $u$ into $P u+Q u$ and project (1.12) into $\mathcal{R}_{0}$ and $\mathcal{N}$, we get the equations

$$
\begin{aligned}
& K(\lambda, v, \psi) \equiv Q F(\lambda, v+\psi)=0, \\
& P F(\lambda, v+\psi)=0
\end{aligned}
$$

where $v=P u$ and $\psi=Q u$. This set of equations is equivalent to the single equation (1.12). Noting that $K\left(\lambda_{0}, 0,0\right)=0$ and $\partial K\left(\lambda_{0}, 0,0\right) / \partial \psi=Q L_{0}$ is an isomorphism from $Q X$ to $Q Y$, we can apply an implicit function theorem to (1.14) and thus solve for $\psi=\psi(\lambda, v)$. We then substitute $\psi$ into (1.15) and get the Liapunov-Schmidt bifurcation equation

$$
P F(\lambda, v+\psi(\lambda, v))=0,
$$

which is a system of $n$ equations; for more details see, for instance, [6,7].

The above scheme cannot be directly applied to the problem (1.1)-(1.6) since the spaces $X$ and $Y$ will vary in $\varepsilon$ in a way which is unknown in advance (as it will depend on the free boundary). Nevertheless, by making a change of variables which maps the free boundary into a fixed boundary, we can state our problem within the framework of the generalized bifurcation theory. The system of PDEs that we obtain in this way, however, has a non-standard structure. As a result, establishing an implicit function theorem requires lengthy and delicate analysis. This will be done in Sections 6 and 7 in the analytic case, i.e. in the case of solution with power expansion in $\varepsilon$. We refer the reader to Remark 6.1 which explains the strategy for establishing the implicit function theorem.

The method we use to establish the existence of analytic bifurcation branches is based on the recent approach by Friedman \& Reitich [5] who established the existence of analytic bifurcation branches for a free boundary problem modelling tumour growth. However, the system (1.1)-(1.6) presents new difficulties due to the following facts: (a) the present system of three PDEs (for $\left.\mu^{+}, \mu^{-}, u\right)$ is more complicated than the system of two PDEs studied in [5]; (b) the present set of free boundary conditions does not allow as strong a priori bounds as in [5] needed to estimate, by induction, the coefficients in the series expansion for the solution. 
Because of (a) we shall require some new properties of zeros of quotients of Bessel functions $I_{m}(x)$ (stated in Appendix A). Because of both (a) and (b), the inductive process (in Section 7) is far more delicate than in [5].

The structure of the paper is as follows. In Section 2 we write down the radial solution of (1.1)-(1.6). In Section 3 we consider the linearized (stationary) problem about the radial solution of (1.1)-(1.6) with free boundary $r=R+\varepsilon \cos l \theta(l \geqslant 2)$ and prove that it has a non-trivial solution if and only if $R=R_{0 l}$ where $R_{0 l}$ is the unique solution of

$$
\frac{I_{0}\left(R_{0 l}\right)}{I_{1}\left(R_{0 l}\right)} \frac{I_{l}\left(R_{0 l}\right)}{I_{l-1}\left(R_{0 l}\right)}=\frac{l+1}{2 l} .
$$

In Section 4 we formally expand the solution of (1.1)-(1.6) into series

$$
\begin{aligned}
& \mu=\mu_{0}(r)+\sum_{n \geqslant 1} \varepsilon^{n} \mu_{n}(r, \theta), \\
& u=u_{0}(r)+\sum_{n \geqslant 1} \varepsilon^{n} u_{n}(r, \theta),
\end{aligned}
$$

with the free boundary and $\beta$ as in (1.7), (1.8), where $\left(\mu_{0}(r), u_{0}(r),\left\{r<R_{0 l}\right\}\right)$ is a radial solution of (1.1)-(1.6). We prove that, subject to the complementary conditions (1.9)-(1.11), the $\mu_{n}, u_{n}, \lambda_{\ln }, \beta_{l, n-1}$ are uniquely determined by induction. To prove convergence of the formal series, however, we need to use another approach.

In Section 5 we transform the free boundary bifurcation problem to one with fixed boundary by the change of variables

$$
r^{\prime}=\frac{r}{R_{0 l}+\varepsilon \cos l \theta+\sum_{n \geqslant 2} \varepsilon^{n} \lambda_{\ln }(\theta)}
$$

For the new system we write down formal series

$$
\begin{aligned}
& \mu=\tilde{\mu}_{0}\left(r^{\prime}\right)+\sum_{n \geqslant 1} \varepsilon^{n} \tilde{\mu}_{n}\left(r^{\prime}, \theta\right), \\
& u=\tilde{u}_{0}\left(r^{\prime}\right)+\sum_{n \geqslant 1} \varepsilon^{n} \tilde{u}_{n}\left(r^{\prime}, \theta\right),
\end{aligned}
$$

with the free boundary and $\beta$ as in (1.7), (1.8). Here again the coefficients $\tilde{\mu}_{n}, \tilde{u}_{n}, \lambda_{\ln }, \beta_{l, n-1}$ are uniquely determined by induction. However, in contrast with the situation in Section 4 , the nonlinear structure of the inductive formulae which define the $\tilde{\mu}_{n}, \tilde{u}_{n}$ and $\lambda_{l, n}, \beta_{l, n-1}$ is much simpler (although the formulae look more complicated). This fact enables us to derive good enough estimates on $\widetilde{\mu}_{n}, \widetilde{u}_{n}, \lambda \ln , \beta_{l, n-1}$ for establishing convergence. The proof of convergence is given in Section 7. Several fundamental estimates needed for this proof are derived in Section 6. The proof in Section 7 requires also some calculus-type estimates on the derivatives of a composite function $\Phi\left(f_{1}(x), \ldots, f_{k}(x)\right)$ in terms of derivatives of both $\Phi\left(u_{1}, \ldots, u_{k}\right)$ (in the $u$ s) and the $f_{j}(x)$ (in $\left.x\right)$. These estimates are proved in Appendix B. In Section 8 we state the main theorem asserting the existence, uniqueness and analyticity (in $(x, \varepsilon)$ ) of the symmetry-breaking bifurcation branches of solutions of (1.1)-(1.6). 


\section{Radial solutions}

We seek stationary solutions $(\mu(r), u(r), R)$ of the system

$$
\begin{aligned}
& \Delta \mu=\chi_{\{r<R\}} \mu \quad \text { for } \quad 0<r<\infty, \\
& \mu(r)=\log r+\text { const. }+\mathrm{O}(1 / r), \quad r \rightarrow \infty, \\
& \mu \text { is continuously differentiable across } r=R, \\
& \Delta u=-\mu \quad \text { if } \quad r<R, \\
& u(R)=0, \\
& u^{\prime}(R)+\beta=0 .
\end{aligned}
$$

The general solution of this system is given by

$$
\mu(r)= \begin{cases}\log r+\lambda I_{0}(R)-\log R, & r>R \\ \lambda I_{0}(r), \quad r \leqslant R & \end{cases}
$$

where $\lambda$ is a constant. Since $\mu^{\prime}(r)$ is continuous across $r=R, 1 / R=\lambda I_{0}^{\prime}(R)=\lambda I_{1}(R)$, so that $\lambda=1 /\left(R I_{1}(R)\right)$. By (2.4), $u(r)+\mu(r)$ is harmonic in $\{r<R\}$, so that $u+\mu=$ const., or

$$
u_{r}=-\mu_{r}=-\lambda I_{0}^{\prime}(r)=-\lambda I_{1}(r)
$$

which implies that $u(r)=\lambda \int_{r}^{R} I_{1}(\xi) \mathrm{d} \xi$ and $\beta=-u^{\prime}(R)=1 / R$. We summarize as follows.

THEOREM 2.1 For any $\beta>0$ there exists a unique radially symmetric solution of (2.1)-(2.6), given by

$$
\begin{gathered}
\mu(r)=\left\{\begin{array}{l}
\log r+\frac{I_{0}(R)}{R I_{1}(R)}-\log R, \quad r>R \\
\frac{I_{0}(r)}{R I_{1}(R)}, \quad r \leqslant R,
\end{array}\right. \\
u(r)=\frac{1}{R I_{1}(R)} \int_{r}^{R} I_{1}(\xi) \mathrm{d} \xi=\frac{I_{0}(R)-I_{0}(r)}{R I_{1}(R)}, \\
R=\frac{1}{\beta} .
\end{gathered}
$$

In what follows we use the notation

$$
[v]_{r=R}=[v](R)=v(R+0)-v(R-0) .
$$

REMARK 2.1 In the time-dependent version of the protocell model [3], the differential equation for $u$ is

$$
c u_{t}-\Delta u=\mu
$$

where $c$ is a positive number, and the Stefan free boundary condition is

$$
V_{n}=-\frac{\partial u}{\partial n}-\beta
$$


By the method developed in [3] one can prove that for any initial data for $u$, there exists a unique radially symmetric solution $\mu(r, t), u(r, t),\{r<R(t)\}$ to this problem for all $t>0$, and, if $c$ is sufficiently small,

$$
\left|R(t)-\frac{1}{\beta}\right|<C \mathrm{e}^{-\alpha t} \quad \forall t>0
$$

where $C, \alpha$ are some positive constants.

\section{The linearized problem}

We want to construct non-radially symmetric stationary solutions of (1.1)-(1.6). To do this we first need to find the bifurcation points $R=R_{0}$, i.e. the values $R=R_{0}$ for which the linearized system of (1.1)-(1.6) about the radially symmetric solution

$$
\left(\mu_{0}(r), u_{0}(r), R_{0}\right)
$$

has a non-trivial solution. The linearization can be made for any mode $l \geqslant 2$ and it corresponds to a perturbation of the free boundary of the form $r=R_{0}+\varepsilon\left(a_{1} \cos l \theta+a_{2} \sin l \theta\right)$. By a translation $\theta \rightarrow \theta+\theta_{0}$ and a scaling of $\varepsilon$ we may take, without loss of generality,

$$
r=R_{0}+\varepsilon \lambda_{1}(\theta), \quad \lambda_{1}(\theta)=\cos l \theta .
$$

Writing

$$
\begin{aligned}
& \mu(r, \theta)=\mu_{0}(r)+\varepsilon \mu_{1}(r) \lambda_{1}(\theta), \\
& u(r, \theta)=u_{0}(r)+\varepsilon u_{1}(r) \lambda_{1}(\theta),
\end{aligned}
$$

we easily derive a system of equations and boundary conditions for $\mu_{1}, u_{1}$ :

$$
\begin{aligned}
& \Delta \mu_{1}-\frac{l^{2}}{r^{2}} \mu_{1}=\chi_{\left\{r<R_{0}\right\}} \mu_{1}, \quad 0<r<\infty, \\
& \mu_{1}=\mathrm{O}(1) \quad \text { as } r \rightarrow \infty, \\
& {\left[\mu_{1}\right]\left(R_{0}\right)=0,} \\
& {\left[\frac{\partial \mu_{1}}{\partial r}\right]\left(R_{0}\right)+\left[\frac{\partial^{2} \mu_{0}}{\partial r^{2}}\right]\left(R_{0}\right)=0}
\end{aligned}
$$

and

$$
\begin{array}{lc}
-\Delta u_{1}+\frac{l^{2}}{r^{2}} u_{1}=\mu_{1} \quad & \text { if } \quad r<R_{0}, \\
u_{1}+\frac{\partial u_{0}}{\partial r}\left(R_{0}\right)=0 \quad \text { if } \quad r=R_{0}, \\
\frac{\partial u_{1}}{\partial r}+\frac{\partial^{2} u_{0}}{\partial r^{2}}\left(R_{0}\right)=0 \quad \text { if } \quad r=R_{0} ;
\end{array}
$$

recall that

$$
\frac{\partial u_{0}}{\partial r}\left(R_{0}\right)=-\beta=-\frac{1}{R_{0}} .
$$


From (3.2), (3.3) we get

$$
\mu_{1}(r)=A I_{l}(r) \quad \text { if } \quad r<R_{0}, \quad \mu_{1}(r)=B r^{-l} \quad \text { if } \quad r>R_{0}
$$

where $A, B$ are constants. From (3.2), (3.6) we see that the function $T=\mu_{1}(r)+u_{1}(r)$ satisfies

$$
T_{r r}+\frac{1}{r} T_{r}-\frac{l^{2}}{r^{2}} T=0 \quad \text { if } \quad r<R_{0},
$$

and therefore $T=C r^{l}$, where $C$ is a constant. Thus

$$
u_{1}(r)=C r^{l}-A I_{l}(r) .
$$

The boundary conditions (3.4), (3.5), (3.7), (3.8) then become

$$
\begin{aligned}
& B R_{0}^{-l}-A I_{l}\left(R_{0}\right)=0, \\
& -B l R_{0}^{-l-1}-A I_{l}^{\prime}\left(R_{0}\right)=\frac{I_{0}\left(R_{0}\right)}{R_{0} I_{1}\left(R_{0}\right)}, \\
& -A I_{l}\left(R_{0}\right)+C R_{0}^{l}=\frac{1}{R_{0}}, \\
& -A I_{l}^{\prime}\left(R_{0}\right)+C l R_{0}^{l-1}=\frac{I_{0}\left(R_{0}\right)}{R_{0} I_{1}\left(R_{0}\right)}-\frac{1}{R_{0}^{2}}
\end{aligned}
$$

and it remains to find $R_{0}$ such that this system has a non-trivial solution $(A, B, C)$.

Using (A.3)-(A.5), the condition on $R_{0}$ reduces to

$$
\frac{I_{0}\left(R_{0}\right)}{I_{1}\left(R_{0}\right)} \frac{I_{l}\left(R_{0}\right)}{I_{l-1}\left(R_{0}\right)}=\frac{l+1}{2 l} .
$$

We now apply Theorem A.1 to conclude the following theorem.

THEOREM 3.1 The linearized system for mode $l$ has a non-trivial solution if and only if $R_{0}$ is the unique solution $R_{0}=R_{0 l}$ of (3.13).

From the previous formulae we find that the solution $\mu_{1}(r), u_{1}(r)$ is given by

$$
\begin{aligned}
& \mu_{1}(r)=\left\{\begin{array}{l}
-\frac{l+1}{2 l} R_{0}^{l-1} r^{-l} \quad \text { if } \quad r>R_{0} \\
-\frac{l+1}{2 l} \frac{I_{l}(r)}{R_{0} I_{l}\left(R_{0}\right)} \quad \text { if } \quad r<R_{0}
\end{array}\right. \\
& u_{1}(r)=\frac{l-1}{2 l} \frac{r^{l}}{R_{0}^{l+1}}+\frac{l+1}{2 l} \frac{I_{l}(r)}{R_{0} I_{l}\left(R_{0}\right)} \quad \text { if } \quad r<R_{0} .
\end{aligned}
$$

REMARK 3.1 For $l=1$, (3.13) holds for all $0<R_{0}<\infty$. The reason is that the problem (1.1)(1.6) is invariant under translation. By translating the centre of the free boundary from $x=(0,0)$ to $x=(0, \varepsilon)$, the solution changes and its new free boundary satisfies

$$
r=R_{0}+\varepsilon \cos \theta+\mathrm{O}\left(\varepsilon^{2}\right) \quad \text { as } \quad \varepsilon \rightarrow 0 .
$$

Since this situation is trivial, we shall not consider the bifurcation associated to $l=1$. On the other hand, if $l \geqslant 2$, the solutions with free boundary given by (1.7), as $\varepsilon \rightarrow 0$, are not radially symmetric with respect to any centre. 
Our goal is to prove that about each bifurcation point $\left(\mu_{1}, u_{1}, R_{0 l}\right)$, there is a bifurcation branch of (symmetry-breaking) analytic solutions with free boundary given by (1.7) and $\beta=\beta(\varepsilon)$ given by $(1.8)$.

\section{Formal solution}

We seek to find symmetry-breaking solutions initiating at $R_{0 l}$ with free boundary

$$
r=R_{0}+f(\theta) \equiv \tilde{f}(\theta) \quad \text { where } \quad R_{0}=R_{0 l}, \quad l \geqslant 2 .
$$

Note that the boundary condition $\partial u / \partial n+\beta=0$ can be written in the form

$$
\tilde{f}(\theta) \frac{\partial u}{\partial r}-\frac{\tilde{f}^{\prime}(\theta)}{\widetilde{f}(\theta)} \frac{\partial u}{\partial \theta}+\beta \sqrt{\tilde{f}^{2}(\theta)+\left(\tilde{f}^{\prime}(\theta)\right)^{2}}=0 .
$$

Since $u(\tilde{f}(\theta), \theta)=0$, we have $u_{r} \tilde{f}^{\prime}+u_{\theta}=0$ on $r=\tilde{f}(\theta)$ and, consequently, the boundary condition (4.2) can also be written in the form

$$
\frac{\partial u}{\partial r}+\beta \frac{R_{0}+f}{\sqrt{\left(R_{0}+f\right)^{2}+f_{\theta}^{2}}}=0 .
$$

In this section we describe a natural formal approach to computing the coefficients in the power series expansions of the bifurcation solutions given by (1.7), (1.8) and (1.18), (1.19). However, as was shown in [5], this approach, surprisingly, is not a good one for actually proving convergence, even for very simple elliptic problems with prescribed boundary that depends analytically on a parameter $\varepsilon$. Therefore, in what follows we shall use another scheme developed in proving convergence; this latter scheme looks more complicated but is nevertheless much easier to work with.

The reason we have included the formal approach of this section in our paper is that it enables us to reduce the compatibility condition which determines the $\beta_{n}$ to a much simpler formula than does the scheme of the subsequent sections. This compatibility condition is based on Lemma 4.2 and is established in Theorem 4.3; it will be used to prove the assertion $\widetilde{\Lambda} \neq 0$ of Lemma 7.1.

In determining (inductively) the coefficients $\lambda_{l m}, \mu_{m}, u_{m}$ in the expansions (1.7), (1.18), (1.19), only powers of $\cos l \theta$ to order $\leqslant m$ will occur. Hence these coefficients will be finite linear combinations of $\cos j l \theta$ with $j \leqslant m$. We can therefore write (1.7) and (1.18), (1.19) more explicitly in the form

$$
\begin{aligned}
& r=R_{0}+\sum_{m=1}^{\infty} \varepsilon^{m} \sum_{j=0}^{m} \tau_{m j} \cos j l \theta \equiv R_{0}+f(\theta, \varepsilon) \\
& \mu=\mu_{0}(r)+\sum_{m=1}^{\infty} \varepsilon^{m} \sum_{j=0}^{m} \mu_{m j}(r) \cos j l \theta \\
& u=u_{0}(r)+\sum_{m=1}^{\infty} \varepsilon^{m} \sum_{j=0}^{m} u_{m j}(r) \cos j l \theta
\end{aligned}
$$


From the differential equation (1.3) we get, for $m \geqslant 1$,

$$
\Delta \mu_{m j}-\frac{j^{2} l^{2}}{r^{2}} \mu_{m j}=\chi_{\left\{r<R_{0}\right\}} \mu_{m j}
$$

so that

$$
\mu_{m j}(r)= \begin{cases}A_{m j} I_{j l}(r) & \text { if } \quad r<R_{0}, \\ B_{m j} r^{-j l} & \text { if } \quad r>R_{0},\end{cases}
$$

where $A_{m j}, B_{m j}$ are constants.

From the differential equations (1.1), (1.4) we find that $\left(\mu_{m j}+u_{m j}\right) \cos j l \theta$ is harmonic in $\left\{r<R_{0}\right\}$ so that

$$
u_{m j}(r)=C_{m j} r^{j l}-A_{m j} I_{j l}(r) \quad \text { if } \quad r<R_{0},
$$

where $C_{m j}$ is a constant.

We now wish to determine the constants $A_{m j}, B_{m j}, C_{m j}, \tau_{m j}$ and $\beta_{m-1}$ so that the boundary conditions (1.3), (1.5), (1.6) are satisfied.

Proceeding by induction we assume that these coefficients have already been determined for $m<n$, and we shall proceed to determine them for $m=n$.

Setting

$$
f_{n}(\theta)=R_{0}+\sum_{m=1}^{n} \varepsilon^{m} \sum_{j=0}^{m} \tau_{m j} \cos j l \theta
$$

the condition (1.3) becomes

$$
\begin{gathered}
{\left[\mu_{0}\right]_{r=f_{n}(\theta)}+\sum_{m=1}^{n} \varepsilon^{m} \sum_{j=0}^{m}\left\{B_{m j} r^{-j l}-A_{m j} I_{j l}(r)\right\}_{r=f_{n}(\theta)} \cos j l \theta=0,} \\
{\left[\frac{\partial \mu_{0}}{\partial r}\right]_{r=f_{n}(\theta)}+\sum_{m=1}^{n} \varepsilon^{m} \sum_{j=0}^{m}\left\{-(j l) B_{m j} r^{-j l-1}-A_{m j} I_{j l}^{\prime}(r)\right\}_{r=f_{n}(\theta)} \cos j l \theta=0 .}
\end{gathered}
$$

The boundary condition (1.5) gives

$$
\left.u_{0}(r)\right|_{r=f_{n}(\theta)}+\sum_{m=1}^{n} \varepsilon^{m} \sum_{j=0}^{m}\left\{C_{m j} r^{j l}-A_{m j} I_{j l}(r)\right\}_{r=f_{n}(\theta)} \cos j l \theta=0 .
$$

Finally, the boundary condition (1.6), written in the form (4.3), becomes

$$
\begin{aligned}
& \left.\frac{\partial u_{0}(r)}{\partial r}\right|_{r=f_{n}(\theta)}+\sum_{m=1}^{n} \varepsilon^{m} \sum_{j=0}^{m}\left\{(j l) C_{m j} r^{j l-1}-A_{m j} I_{j l}^{\prime}(r)\right\}_{r=f_{n}(\theta)} \cos j l \theta \\
& \quad+\left(\beta_{0}+\sum_{m=2}^{n} \varepsilon^{m} \beta_{m}\right) \sqrt{\frac{1}{1+\left\{f_{n}^{\prime}(\theta) / f_{n}(\theta)\right\}^{2}}}=0 .
\end{aligned}
$$


Using the fact that $\left[\mu_{0}\right]\left(R_{0}\right)=\left[\frac{\partial \mu_{0}}{\partial r}\right]\left(R_{0}\right)=0$, we find from (4.11), (4.12), by equating the coefficients of $\varepsilon^{n} \cos j l \theta$, that

$$
\begin{aligned}
& B_{n j} R_{0}^{-j l}-A_{n j} I_{j l}\left(R_{0}\right)=F_{n j}^{1}, \\
& -(j l) B_{n j} R_{0}^{-j l-1}-A_{n j} I_{j l}^{\prime}\left(R_{0}\right)+\tau_{n j}\left[\frac{\partial^{2} \mu_{0}}{\partial r^{2}}\right]\left(R_{0}\right)=F_{n j}^{2},
\end{aligned}
$$

where $F_{n j}^{1}, F_{n j}^{2}$ are determined by the inductive assumption, i.e. they are given in terms of the $A_{m k}, B_{m k}, C_{m k}, \tau_{m k}, \beta_{m-1}$ for $m \leqslant n-1$; the parameter $\beta_{n-1}$, however, has not yet been determined.

Similarly, from (4.13) we obtain, using the relations $u_{0}\left(R_{0}\right)=0, \partial u_{0} / \partial r\left(R_{0}\right)=-\beta_{0}=$ $-1 / R_{0}$,

$$
-A_{n j} I_{j l}\left(R_{0}\right)+C_{n j} R_{0}^{j l}-\frac{1}{R_{0}} \tau_{n j}=F_{n j}^{3}
$$

where $F_{n j}^{3}$ is determined by the inductive assumption, i.e. it is given in terms of $A_{m k}, B_{m k}, C_{m k}, \tau_{m k}$ and $\beta_{m-1}$ for $m \leqslant n-1$.

Finally, from (4.14) we get

$$
-A_{n j} I_{j l}\left(R_{0}\right)+(j l) C_{n j} R_{0}^{j l-1}+\tau_{n j} \frac{\partial^{2} u_{0}\left(R_{0}\right)}{\partial r^{2}}=\widetilde{F}_{n j}^{4}-\beta_{n} \delta_{j 0} \equiv F_{n j}^{4},
$$

where $\widetilde{F}_{n j}^{4}$ is given by the inductive assumption as the preceding $F_{n j}^{i}$. The coefficients matrix of the linear system (4.15)-(4.18), for fixed $j$, is

$$
T_{j l}=\left(\begin{array}{cccc}
-I_{j l}\left(R_{0}\right) & R_{0}^{-j l} & 0 & 0 \\
-I_{j l}^{\prime}\left(R_{0}\right) & (-j l) R_{0}^{-j l-1} & 0 & {\left[\frac{\partial^{2} \mu_{0}}{\partial r^{2}}\right]\left(R_{0}\right)} \\
-I_{j l}\left(R_{0}\right) & 0 & R_{0}^{j l} & -\frac{1}{R_{0}} \\
-I_{j l}^{\prime}\left(R_{0}\right) & 0 & (j l) R_{0}^{j l-1} & \frac{\partial^{2} u_{0}\left(R_{0}\right)}{\partial r^{2}}
\end{array}\right) .
$$

Noting that

$$
\alpha \equiv-\left[\frac{\partial^{2} \mu_{0}}{\partial r^{2}}\right]\left(R_{0}\right)=\frac{I_{0}\left(R_{0}\right)}{R_{0} I_{1}\left(R_{0}\right)}, \quad \gamma \equiv \frac{\partial^{2} u_{0}\left(R_{0}\right)}{\partial r^{2}}=\frac{1}{R_{0}^{2}}-\alpha,
$$

and using (A.3), (A.4), we easily obtain, for $m=j l$, the formula

$$
\operatorname{det} T_{m}=2 m \frac{I_{m-1}\left(R_{0}\right)}{R_{0}^{2}}\left(\frac{I_{0}\left(R_{0}\right)}{I_{1}\left(R_{0}\right)} \frac{I_{m}\left(R_{0}\right)}{I_{m-1}\left(R_{0}\right)}-\frac{m+1}{2 m}\right) .
$$

Using Theorem A.2 we conclude as follows.

THEOREM 4.1 There holds:

$$
\operatorname{det} T_{j l} \neq 0 \quad \text { if } j \neq 1 ; \quad \operatorname{det} T_{l}=0 .
$$


Equation $\operatorname{det} T_{l}=0$ is just (3.13), the solvability condition for the linearized problem. Equation $\operatorname{det} T_{j l} \neq 0$ for $j \neq 1$ implies the solvability of the equations for the coefficients in (4.4)-(4.6) for any $j \neq 1$. Thus it remains to solve the system (4.15)-(4.18) in case $j=1$. Here we shall need to use the parameter $\beta_{n-1}$ to ensure solvability.

REMARK 4.1 For $n=2$ mode $l$ terms do not appear in the $F_{n j}^{0}$ and thus the system (4.15)-(4.18) has a unique solution. However, in this special case, we necessarily have that $\beta_{1}=0$. Indeed, if $n=1$ then $F_{n j}^{1}=0, F_{n j}^{2}=0, F_{n j}^{3}=0$ and $\widetilde{F}_{n j}^{4}=0$, and since the linearized solution is of mode $l$, also $A_{1 j}=B_{1 j}=C_{1 j}=\tau_{1 j}=0$ if $j=0$. Equation (4.18) then implies that $\beta_{1}=0$. This fact can also be proved in another way. Denote by $\Omega_{\varepsilon}$ the domain bounded by $r=R_{0}+\varepsilon \cos l \theta+\mathrm{O}\left(\varepsilon^{2}\right)$. Then

$$
\beta \int_{\partial \Omega_{\varepsilon}} 1=-\int_{\partial \Omega_{\varepsilon}} \frac{\partial u}{\partial n}=-\int_{\Omega_{\varepsilon}} \Delta u=\int_{\Omega_{\varepsilon}} \mu
$$

where $\mu=\mu_{0}(r)+\varepsilon \mu_{1}(r) \cos l \theta+\mathrm{O}\left(\varepsilon^{2}\right)$. Since

$$
\int_{\partial \Omega_{\varepsilon}} 1=2 \pi R_{0}+\mathrm{O}\left(\varepsilon^{2}\right), \quad \int_{\Omega_{\varepsilon}} \mu(r)=\int_{\Omega_{0}} \mu_{0}(r)+\mathrm{O}\left(\varepsilon^{2}\right),
$$

writing $\beta=\beta_{0}+\varepsilon \beta_{1}+\mathrm{O}\left(\varepsilon^{2}\right)$ we get

$$
\left(\beta_{0}+\varepsilon \beta_{1}+\mathrm{O}\left(\varepsilon^{2}\right)\right)\left(2 \pi R_{0}+\mathrm{O}\left(\varepsilon^{2}\right)\right)=\int_{\Omega_{0}} \mu_{0}(r)+\mathrm{O}\left(\varepsilon^{2}\right)
$$

which implies that $\beta_{1}=0$.

Introducing the vector notation

$$
\boldsymbol{X}_{n j}=\left(\begin{array}{c}
A_{n j} \\
B_{n j} \\
C_{n j} \\
\tau_{n j}
\end{array}\right), \quad \boldsymbol{F}_{n j}=\left(\begin{array}{c}
F_{n j}^{1} \\
F_{n j}^{2} \\
F_{n j}^{3} \\
F_{n j}^{4}
\end{array}\right),
$$

we can write the system (4.15)-(4.18) for $j=1$ in the form

$$
T_{l} \boldsymbol{X}_{n 1}=\boldsymbol{F}_{n 1} .
$$

This system is solvable if and only if the augmented matrix has the same rank as $T_{l}$, i.e. if and only if

$$
\operatorname{det}\left(\begin{array}{cccc}
F_{n 1}^{1} & R_{0}^{-l} & 0 & 0 \\
F_{n 1}^{2} & -l R_{0}^{-l-1} & 0 & -\alpha \\
F_{n 1}^{3} & 0 & R_{0}^{l} & -\frac{1}{R_{0}} \\
F_{n 1}^{4} & 0 & l R_{0}^{l} & \gamma
\end{array}\right)=0 .
$$

Adding $l / R_{0}$ times the first row to the second row and $(-l) / R_{0}$ times the third row to the fourth row, the above condition reduces to

$$
\operatorname{det}\left(\begin{array}{cc}
\frac{l}{R_{0}} F_{n 1}^{1}+F_{n 1}^{2} & -\alpha \\
-\frac{l}{R_{0}} F_{n 1}^{3}+F_{n 1}^{4} & \frac{l+1}{R_{0}^{2}}-\alpha
\end{array}\right)=0
$$

(here we used the definitions of $\alpha, \gamma$ in (4.19)). Thus we have the following lemma. 
LEMMA 4.2 The system (4.20) has a solution if and only if

$$
\left(\frac{l+1}{R_{0}^{2}}-\alpha\right)\left(\frac{l}{R_{0}} F_{n 1}^{1}+F_{n 1}^{2}\right)+\alpha\left(-\frac{l}{R_{0}} F_{n 1}^{3}+F_{n 1}^{4}\right)=0 .
$$

We want to prove that $\beta_{n-1}$ can be uniquely determined so that (4.21) holds. We first have to examine how $\beta_{n-1}$ enters into $X_{n-1, j}$. It clearly appears only in the last equation for $X_{n-1,0}$ (see (4.18) with $n$ replaced by $n-1)$ :

$$
T_{0} \boldsymbol{X}_{n-1,0}=\boldsymbol{F}_{n-1,0}-\beta_{n-1}\left(\begin{array}{l}
0 \\
0 \\
0 \\
1
\end{array}\right) .
$$

We can therefore write

$$
\boldsymbol{X}_{n-1,0}=\widehat{\boldsymbol{X}}_{n-1,0}+\overline{\boldsymbol{X}}_{n-1,0}
$$

where $\widehat{\boldsymbol{X}}_{n-1,0}$ does not depend on $\beta_{n-1}$, and, setting

$$
\overline{\boldsymbol{X}}_{n-1,0}=\left(\begin{array}{l}
\bar{A}_{n-1} \\
\bar{B}_{n-1} \\
\bar{C}_{n-1} \\
\bar{\tau}_{n-1}
\end{array}\right)=\beta_{n-1} \overline{\boldsymbol{X}}, \quad \overline{\boldsymbol{X}}=\left(\begin{array}{l}
\bar{A} \\
\bar{B} \\
\bar{C} \\
\bar{\tau}
\end{array}\right),
$$

there holds: $T_{0} \overline{\boldsymbol{X}}=-(0,0,0,1)^{T}$. One can easily compute that

$$
\bar{A}=R_{0} \frac{I_{0}\left(R_{0}\right)}{I_{1}^{2}\left(R_{0}\right)}, \quad \bar{B}=R_{0} \frac{I_{0}^{2}\left(R_{0}\right)}{I_{1}^{2}\left(R_{0}\right)}, \quad \bar{C}=R_{0}\left(\frac{I_{0}^{2}\left(R_{0}\right)}{I_{1}^{2}\left(R_{0}\right)}-1\right), \quad \bar{\tau}=-R_{0}^{2} .
$$

We now return to (4.20) and write

$$
\boldsymbol{F}_{n 1}=\widehat{\boldsymbol{F}}_{n 1}+\overline{\boldsymbol{F}}_{n 1} \beta_{n-1},
$$

where $\widehat{\boldsymbol{F}}_{n 1}$ and $\widehat{\boldsymbol{F}}_{n 2}$ are independent of $\beta_{n-1}$. We need to compute $\overline{\boldsymbol{F}}_{n 1}=\left(\bar{F}^{1}, \bar{F}^{2}, \bar{F}^{3}, \bar{F}^{4}\right)^{T}$.

To do that we write (see (4.10))

$$
f_{n}(\theta)=R_{0}+\varepsilon \cos l \theta+\varepsilon^{n-1} \bar{\tau}_{n-1}+\mathrm{O}\left(\varepsilon^{2}\right)=R_{0}+g(\theta)
$$

where $\bar{\tau}_{n-1}=\tau_{n-1,0}$. Then

$$
\begin{aligned}
& g=\varepsilon \cos l \theta+\varepsilon^{n-1} \bar{\tau}_{n-1}+\mathrm{O}\left(\varepsilon^{2}\right), \\
& (g)^{2}=2 \varepsilon^{n} \cos l \theta \cdot \bar{\tau}_{n-1}+\mathrm{O}\left(\varepsilon^{2}\right), \quad(g)^{k}=\mathrm{O}\left(\varepsilon^{2}\right) \quad(k \geqslant 3) .
\end{aligned}
$$

Here $\mathrm{O}\left(\varepsilon^{2}\right)$ does not depend explicitly on $\bar{\tau}_{n-1}$ and terms of order $\varepsilon^{n+1}$ have been discarded; these conventions are also used in what follows.

We also have

$$
\begin{aligned}
& \left(f_{n}\right)^{k}=R_{0}^{k}+k R_{0}^{k-1} \varepsilon^{n-1} \bar{\tau}_{n-1}+k R_{0}^{k-1} \varepsilon \cos l \theta+\mathrm{O}\left(\varepsilon^{2}\right)+\mathrm{O}_{\bar{\tau}}\left(\varepsilon^{n}\right), \\
& \left(f_{n}\right)^{-k}=R_{0}^{-k}-k R_{0}^{-k-1} \varepsilon^{n-1} \bar{\tau}_{n-1}-k R_{0}^{-k-1} \varepsilon \cos l \theta+\mathrm{O}\left(\varepsilon^{2}\right)+\mathrm{O}_{\bar{\tau}}\left(\varepsilon^{n}\right)
\end{aligned}
$$


where $\mathrm{O}_{\bar{\tau}}\left(\varepsilon^{n}\right)$ may depend on $\bar{\tau}_{n-1}$.

From (4.26) we obtain

$$
\begin{aligned}
& \left.I_{m}(r)\right|_{r=f_{n}(\theta)}=I_{m}\left(R_{0}\right)+I_{m}^{\prime}\left(R_{0}\right) \varepsilon^{n-1} \bar{\tau}_{n-1}+I_{m}^{\prime}\left(R_{0}\right) \varepsilon \cos l \theta+\mathrm{O}\left(\varepsilon^{2}\right)+\mathrm{O}_{\bar{\tau}}\left(\varepsilon^{n}\right), \\
& \left.I_{m}^{\prime}(r)\right|_{r=f_{n}(\theta)}=I_{m}^{\prime}\left(R_{0}\right)+I_{m}^{\prime \prime}\left(R_{0}\right) \varepsilon^{n-1} \bar{\tau}_{n-1}+I_{m}^{\prime}\left(R_{0}\right) \varepsilon \cos l \theta+\mathrm{O}\left(\varepsilon^{2}\right)+\mathrm{O}_{\bar{\tau}}\left(\varepsilon^{n}\right) .
\end{aligned}
$$

We proceed to compute the first component $\bar{F}^{1}$ of $\overline{\boldsymbol{F}}_{n 1}$. For this we need to identify in (4.11) the terms of the form $\lambda \varepsilon^{n} \cos l \theta$ where $\lambda$ depends only on $\bar{A}_{n-1}, \bar{B}_{n-1}, \bar{C}_{n-1}, \bar{\tau}_{n-1}$; all other terms are irrelevant and will be collectively designated by '...'.

Using (4.26) we find that the first term on the left-hand side of (4.11) is of the form

$$
\left[\mu_{0}\right]_{r=f_{n}(\theta)}=\frac{1}{2} g^{2}\left[\frac{\partial^{2} \mu_{0}}{\partial r^{2}}\right]\left(R_{0}\right)+\cdots=\left[\frac{\partial^{2} \mu_{0}}{\partial r^{2}}\right]\left(R_{0}\right) \varepsilon^{n} \cos l \theta \cdot \bar{\tau}_{n-1}+\cdots .
$$

Using (4.28) we see that from the series $\sum \varepsilon^{m} \sum B_{m j} r^{-j l} \cos j l \theta$ we can get $\varepsilon^{n} \cos l \theta \cdot \bar{\tau}_{n-1}$ only if $m=1$ and (necessarily) $j=1$. We thus get

$$
-\frac{l}{R_{0}^{l+1}} B_{11} \varepsilon^{n} \cos l \theta \cdot \bar{\tau}_{n-1} .
$$

The coefficient $\bar{B}_{n-1}$ does not come with $\varepsilon^{n} \cos l \theta$. Similarly, using (4.29) we get from $-\sum \varepsilon^{m} \sum A_{m j} I_{j l}(r) \cos j l$ the term

$$
-I_{l}^{\prime}\left(R_{0}\right) A_{11} \varepsilon^{n} \cos l \theta \cdot \bar{\tau}_{n-1} .
$$

We also observe that $\bar{A}_{n-1}$ appears when $m=n-1, j=0$ in the form

$$
-\varepsilon^{n} \cos l \theta \cdot \bar{A}_{n-1} I_{0}^{\prime}\left(R_{0}\right) \text {. }
$$

Combining (4.31a)-(4.31d) we conclude that

$$
-\bar{F}^{1}=\bar{\tau}_{n-1}\left(-\alpha-\frac{l}{R_{0}^{l+1}} B_{11}-I_{l}^{\prime}\left(R_{0}\right) A_{11}\right)-\bar{A}_{n-1} I_{1}\left(R_{0}\right) .
$$

Next we consider (4.12). From the first term or the left-hand side we get

$$
\left[\frac{\partial^{3} \mu_{0}}{\partial r^{3}}\right]\left(R_{0}\right) \varepsilon^{n} \cos l \theta \cdot \bar{\tau}_{n-1} .
$$

From the sum of the $B_{m j}$ we can get $\varepsilon^{n} \cos l \theta \cdot \bar{\tau}_{n-1}$ only if $m=1$ and $j=1$; the resulting term is

$$
\frac{l+1}{R_{0}^{l+2}} l B_{11} \varepsilon^{n} \cos l \theta \cdot \bar{\tau}_{n-1} .
$$

From the sum of the $A_{m j} I_{j l}^{\prime}$ we get $\varepsilon^{n} \cos l \theta \cdot \bar{\tau}_{n-1}$ only if $m=1$ and $j=1$. Using (4.30) we find that this gives the term

$$
-I_{l}^{\prime \prime}\left(R_{0}\right) A_{11} \varepsilon^{n} \cos l \theta \cdot \bar{\tau}_{n-1} .
$$


Finally, $\bar{A}_{n-1}$ appears (when $m=n-1, j=0$ ) in the form

$$
-\varepsilon^{n} \cos l \theta \cdot \bar{A}_{n-1} \cdot I_{1}^{\prime}\left(R_{0}\right) \text {. }
$$

Combining (4.33a)-(4.33d) we see that

$$
-\bar{F}^{2}=\bar{\tau}_{n-1}\left\{\left[\frac{\partial^{3} \mu_{0}}{\partial r^{3}}\right]\left(R_{0}\right)+\frac{l(l+1)}{R_{0}^{l+2}} B_{11}-I_{l}^{\prime \prime}\left(R_{0}\right) A_{11}\right\}-\bar{A}_{n-1} I_{1}^{\prime}\left(R_{0}\right) .
$$

Next, by the same analysis as for (4.11), from the first term in (4.13) we obtain

$$
\frac{\partial^{2} u_{0}}{\partial r^{2}}\left(R_{0}\right) \varepsilon^{n} \cos l \theta \cdot \bar{\tau}_{n-1},
$$

and from the remaining terms we obtain

$$
C_{11} l R_{0}^{l-1} \varepsilon^{n} \cos l \theta \cdot \bar{\tau}_{n-1}, \quad-I_{l}^{\prime}\left(R_{0}\right) A_{11} \varepsilon^{n} \cos l \theta \cdot \bar{\tau}_{n-1}, \quad-\varepsilon^{n} \cos l \theta \cdot \bar{A}_{n-1} I_{0}^{\prime}\left(R_{0}\right),
$$

so that

$$
-\bar{F}^{3}=\bar{\tau}_{n-1}\left[\gamma+C_{11} l R_{0}^{l-1}-I_{l}^{\prime}\left(R_{0}\right) A_{11}\right]-\bar{A}_{n-1} I_{1}\left(R_{0}\right) .
$$

Finally we consider (4.14). Computations as above show that we collect the terms

$$
\frac{\partial^{3} u_{0}}{\partial r^{3}}\left(R_{0}\right) \varepsilon^{n} \cos l \theta \cdot \bar{\tau}_{n-1}
$$

from $\partial u_{0} / \partial r$ and

$$
l(l-1) C_{11} R_{0}^{l-2} \varepsilon^{n} \cos l \theta \cdot \bar{\tau}_{n-1}, \quad-I_{l}^{\prime \prime}\left(R_{0}\right) A_{11} \varepsilon^{n} \cos l \theta \cdot \bar{\tau}_{n-1}, \quad-I_{1}^{\prime}\left(R_{0}\right) \varepsilon^{n} \cos l \theta \cdot \bar{A}_{n-1}
$$

from the double sum in (4.14).

Since $f^{\prime}(\theta)$ does not contain $\bar{\tau}_{n-1},\left\{1+\left(f^{\prime}(\theta) / f(\theta)\right)^{2}\right\}^{-1 / 2}$ does not contain terms of the form const. $\varepsilon^{n} \cos l \theta \cdot \bar{\tau}_{n-1}$. From (4.14) it then follows that

$$
-\bar{F}^{4}=\bar{\tau}_{n-1}\left\{\frac{\partial^{3} u_{0}}{\partial r^{3}}\left(R_{0}\right)+l(l-1) C_{11} R_{0}^{l-2}-I_{l}^{\prime \prime}\left(R_{0}\right) A_{11}\right\}-\bar{A}_{n-1} I_{1}^{\prime}\left(R_{0}\right) .
$$

We shall next compute more explicitly the coefficients $\bar{F}^{j}$, making use of the formula

$$
A_{11}=-\frac{l+1}{2 l} \frac{1}{R_{0} I_{l}\left(R_{0}\right)}, \quad B_{11}=-\frac{l+1}{2 l} R_{0}^{l-1}, \quad C_{11}=\frac{l-1}{2 l} \frac{1}{R_{0}^{l+1}}
$$

which follow from (3.14), (3.15) (after using also (3.13)).

Dropping the independent variable $R_{0}$ in the various Bessel functions, we find that the coefficient of $\bar{\tau}_{n-1}$ in $\bar{F}^{1}$ is equal to

$$
\begin{aligned}
\frac{I_{0}}{R_{0} I_{1}}+I_{l}^{\prime} A_{11}+\frac{l}{R_{0}^{l+1}} B_{11} & =\frac{I_{0}}{R_{0} I_{1}}-I_{l}^{\prime} \frac{l+1}{2 l} \frac{1}{R_{0} I_{l}}-\frac{l}{R_{0}^{l+1}} \frac{l+1}{2 l} R_{0}^{l-1} \\
& =\frac{I_{0}}{R_{0} I_{1}}-\frac{l+1}{2 l R_{0} I_{l}}\left[I_{l}^{\prime}+\frac{l}{R_{0}} I_{l}\right]=0
\end{aligned}
$$


by (A.3) and (3.13). Thus, by (4.32),

$$
\bar{F}^{1}=I_{1}\left(R_{0}\right) \bar{A}_{n-1} .
$$

The coefficient of $\bar{\tau}_{n-1}$ in $-\bar{F}^{4}$ is equal to

$$
\begin{aligned}
& {\left[\frac{\partial^{3} \mu_{0}}{\partial r^{3}}\right]\left(R_{0}\right)+\frac{l(l+1)}{R_{0}^{l+2}} B_{11}-A_{11} I_{l}^{\prime \prime}} \\
& \quad=\frac{I_{0}}{R_{0}^{2} I_{1}}-\frac{1}{R_{0}}-\frac{l(l+1)}{R_{0}^{l+2}} \frac{l+1}{2 l} R_{0}^{l-1}+\frac{l+1}{2 l} \frac{I_{l}^{\prime \prime}}{R_{0} I_{l}} \quad \text { (by direct computation) } \\
& \quad=\frac{I_{0}}{R_{0}^{2} I_{1}}-\frac{1}{R_{0}}-\frac{(l+1)^{2}}{2} \frac{1}{R_{0}^{3}}-\frac{l+1}{2 l} \frac{I_{l}^{\prime}}{R_{0}^{2} I_{l}}+\frac{l+1}{2 l R_{0}}\left(1+\frac{l^{2}}{R_{0}^{2}}\right) \quad \text { (by (A.1)) } \\
& \quad=\frac{I_{0}}{R_{0}^{2} I_{1}}-\frac{l-1}{2 l} \frac{1}{R_{0}}-\frac{l+1}{2 R_{0}^{2} l I_{l}}\left(I_{l}^{\prime}+\frac{l}{R_{0}} I_{l}\right)=-\frac{l-1}{2 l} \frac{1}{R_{0}},
\end{aligned}
$$

by (A.3) and (3.13). Hence

$$
\bar{F}^{2}=\frac{l-1}{2 l} \frac{1}{R_{0}} \bar{\tau}_{n-1}+I_{1}^{\prime}\left(R_{0}\right) \bar{A}_{n-1} .
$$

Next, the coefficient of $\bar{\tau}_{n-1}$ in $-\bar{F}^{3}$ is equal to

$$
\begin{aligned}
& \left(\frac{1}{R_{0}^{2}}-\frac{I_{0}}{R_{0} I_{1}}\right)+\frac{1}{R_{0}^{l+1}} \frac{l-1}{2 l} l R_{0}^{l-1}+\frac{I_{l}^{\prime}}{I_{l}} \frac{l+1}{2 l R_{0}} \\
& =-\frac{I_{0}}{R_{0} I_{1}}+\frac{l+1}{2 l R_{0} I_{l}}\left(I_{l}^{\prime}+\frac{l}{R_{0}} I_{l}\right)=0,
\end{aligned}
$$

by (A.3) and (3.13), so that

$$
\bar{F}^{3}=I_{1}\left(R_{0}\right) \bar{A}_{n-1} .
$$

Finally, the coefficient of $\bar{\tau}_{n-1}$ in $-\bar{F}^{4}$ is equal to

$$
\begin{aligned}
\frac{\partial^{3} u_{0}}{\partial r^{3}} & \left(R_{0}\right)+l(l-1) C_{11} R_{0}^{l-2}-A_{11} I_{l}^{\prime \prime} \\
& =\frac{\partial^{3} u_{0}}{\partial r^{3}}\left(R_{0}\right)+l(l-1) \frac{l-1}{2 l} \frac{1}{R_{0}^{l+1}} R_{0}^{l-2}+I_{l}^{\prime \prime} \frac{l+1}{2 l} \frac{1}{R_{0} I_{l}} \\
& =-\frac{2}{R_{0}^{3}}-\frac{1}{R_{0}}+\frac{I_{0}}{R_{0}^{2} I_{1}}+\frac{(l-1)^{2}}{2} \frac{1}{R_{0}^{3}}+\frac{l+1}{2 l R_{0}}\left[\left(1+\frac{l^{2}}{R_{0}^{2}}\right)-\frac{I_{l}^{\prime}}{R_{0} I_{l}}\right] \quad \text { (by (A.1)) } \\
& =\frac{1}{R_{0}^{3}}\left(-2+\frac{(l-1)^{2}}{2}+\frac{l(l+1)}{2}\right)-\frac{l-1}{2 l R_{0}}+\frac{I_{0}}{R_{0}^{2} I_{1}}-\frac{l+1}{2 l R_{0}^{2}}\left(\frac{I_{l-1}}{I_{l}}-\frac{l}{R_{0}}\right) \quad \text { (by (A.3)) } \\
& =\frac{l^{2}-1}{R_{0}^{3}}-\frac{l-1}{2 l R_{0}}
\end{aligned}
$$


by (3.13).

We conclude that

$$
\bar{F}^{4}=-\left[\frac{l^{2}-1}{R_{0}^{3}}-\frac{l-1}{2 l R_{0}}\right] \bar{\tau}_{n-1}+\bar{A}_{n-1} I_{1}^{\prime}\left(R_{0}\right)
$$

Since, by (4.23),

$$
\bar{\tau}_{n-1}=-\frac{R_{0} I_{1}^{2}\left(R_{0}\right)}{I_{0}\left(R_{0}\right)} \bar{A}_{n-1}
$$

we can rewrite (4.39) and (4.41) in the form

$$
\begin{gathered}
\bar{F}^{2}=\left[-\frac{l-1}{2 l} \cdot \frac{I_{1}^{2}\left(R_{0}\right)}{I_{0}\left(R_{0}\right)}+I_{1}^{\prime}\left(R_{0}\right)\right] \bar{A}_{n-1}, \\
\bar{F}^{4}=\left\{\left[\frac{l^{2}-1}{R_{0}^{3}}-\frac{l-1}{2 l R_{0}}\right] \frac{R_{0} I_{1}^{2}\left(R_{0}\right)}{I_{0}\left(R_{0}\right)}+I_{1}^{\prime}\left(R_{0}\right)\right\} \bar{A}_{n-1} .
\end{gathered}
$$

Substituting (4.38), (4.42), (4.40) and (4.43) into the solvability condition (4.21) and using the relation $I_{1}^{\prime}=I_{0}-I_{1} / R_{0}$, we get

$$
\Lambda\left(R_{0}\right) \beta_{n-1}=\widetilde{F}_{n}
$$

where $\widetilde{F}_{n}$ depends only on $A_{m j}, B_{m j}, C_{m j}, \tau_{m j}, \beta_{m-1}$ for $m \leqslant n-1$, and

$$
\Lambda(R)=\frac{l-1}{R} I_{0}(R)\left\{\frac{2(l+1)}{R}\left(\frac{I_{1}(R)}{I_{0}(R)}\right)-\frac{l+1}{2 l}\left(\frac{I_{1}(R)}{I_{0}(R)}\right)^{2}-1\right\} .
$$

We now prove the following theorem.

THEOREM 4.3

$$
\Lambda\left(R_{0}\right)<0 .
$$

Consequently, equation (4.44) defines $\beta_{n-1}$ uniquely in terms of $A_{m j}, B_{m j}, C_{m j}, \tau_{m j}$ and $\beta_{m-1}$ for all $m \leqslant n-1$. Thus the asymptotic expansion can be developed for all $n$, and it is easy to see that it formally defines a solution to the free boundary problem up to any order of precision $\varepsilon^{n}$.

ProOF OF THEOREM 4.1 Introduce the functions

$$
V(x)=\frac{I_{m}(x)}{I_{m-1}(x)}, \quad G(x)=\frac{I_{1}(x)}{I_{0}(x)} .
$$

By (A.1)-(A.3) we find that

$$
V^{\prime}+V^{2}+\frac{2 m-1}{x} V=1, \quad G^{\prime}+G^{2}+\frac{1}{x} G=1 .
$$

so that

$$
\frac{V^{\prime}(x)}{V(x)}-\frac{G^{\prime}(x)}{G(x)}=\frac{G(x)}{V(x)} \frac{\mathrm{d}}{\mathrm{d} x}\left(\frac{V(x)}{G(x)}\right)>0
$$


by Theorem A.1. Substituting $V^{\prime}, G^{\prime}$ from (4.47) into this inequality, and using (3.13), we find that, at $x=R_{0}$,

$$
\begin{aligned}
0 & <\left(\frac{1}{V}-V-\frac{2 m-1}{x}\right)-\left(\frac{1}{G}-G-\frac{1}{x}\right) \\
& =\left(\frac{2 m}{m+1} \frac{1}{G}-\frac{m+1}{2 m} G-\frac{2 m-1}{x}\right)-\left(\frac{1}{G}-G-\frac{1}{x}\right) \\
& =\frac{m-1}{m+1} \frac{1}{G}+\frac{m-1}{2 m} G-\frac{2(m-1)}{x} \\
& =\frac{m-1}{m+1} \frac{1}{G}\left[1+\frac{m+1}{2 m} G^{2}-\frac{2(m+1)}{x} G\right],
\end{aligned}
$$

so that $\Lambda\left(R_{0}\right)<0$.

REMARK 4.2 The mode $l$ solution is uniquely determined up to a multiple of a special solution of the homogeneous system (4.15)-(4.18). It follows that by imposing the condition $\tau_{n l}=0$ (i.e. $\int_{0}^{2 \pi} \lambda \ln (\theta) \cos l \theta \mathrm{d} \theta=0$ ) we get a unique solution to (4.15)-(4.18). We have thus proved the following theorem.

THEOREM 4.4 There exists a unique formal power series solution (4.4)-(4.6), (1.8) of (1.1)-(1.6) subject to the conditions (1.9)-(1.11).

\section{A change of variables}

There is a serious difficulty in proving directly that the power series of the formal solution asserted in Theorem 4.4 is convergent (see [5]). We therefore proceed indirectly, by first transforming the free boundary problem into a problem in a fixed domain. We perform the change of variables

$$
r^{\prime}=r /\left(R_{0}+f(\theta, \varepsilon)\right),
$$

under which the original problem is reduced to a problem in a disc $\left\{r^{\prime}<1\right\}$. For simplicity, we shall still use $r$ for the new variable (instead of $r^{\prime}$ ) and set

$$
\Delta=\frac{1}{\left(R_{0}+f(\theta, \varepsilon)\right)^{2}} \mathcal{L}(D),
$$

where

$$
f(\theta, \varepsilon))=\sum_{j=1}^{\infty} \varepsilon^{j} \lambda_{j}(\theta),
$$

and

$$
\begin{aligned}
\mathcal{L}(D)= & \frac{\partial^{2}}{\partial r^{2}}+\frac{1}{r} \frac{\partial}{\partial r}+\frac{1}{r^{2}} \frac{\partial^{2}}{\partial \theta^{2}}-\frac{f_{\theta \theta}}{R_{0}+f} \frac{1}{r} \frac{\partial}{\partial r} \\
& -\frac{2 f_{\theta}}{R_{0}+f} \frac{1}{r} \frac{\partial^{2}}{\partial \theta \partial r}+\frac{2 f_{\theta}^{2}}{\left(R_{0}+f\right)^{2}} \frac{1}{r} \frac{\partial}{\partial r}+\frac{f_{\theta}^{2}}{\left(R_{0}+f\right)^{2}} \frac{\partial^{2}}{\partial r^{2}} .
\end{aligned}
$$


Then, in the new coordinates, the system becomes

$$
\begin{aligned}
& \mathcal{L}(D) \mu=\chi_{\{r<1\}}\left(R_{0}+f\right)^{2} \mu \quad \text { in }\{r<\infty\}, \\
& \mathcal{L}(D) u=\left(R_{0}+f\right)^{2} u \quad \text { in }\{r<1\},
\end{aligned}
$$

with the boundary conditions

$$
\begin{aligned}
& u=0 \quad \text { on } r=1, \\
& {[\mu]=\left[\frac{\partial \mu}{\partial r}\right]=0 \quad \text { on } r=1,} \\
& \frac{\partial u}{\partial r}+\beta \frac{\left(R_{0}+f\right)^{2}}{\left[\left(R_{0}+f\right)^{2}+f_{\theta}^{2}\right]^{1 / 2}}=0 \quad \text { on } r=1 .
\end{aligned}
$$

As before, we seek solutions of the form

$$
\begin{aligned}
& u=u_{0}(r)+\sum_{j=1}^{\infty} \varepsilon^{j} u_{j}(r, \theta), \\
& \mu=\mu_{0}(r)+\sum_{j=1}^{\infty} \varepsilon^{j} \mu_{j}(r, \theta), \\
& f(\theta, \varepsilon)=R_{0}+\varepsilon \cos l \theta+\sum_{j=2}^{\infty} \varepsilon^{j} \lambda_{j}(\theta), \\
& \beta=\beta_{0}+\sum_{j=1}^{\infty} \varepsilon^{j} \beta_{j} .
\end{aligned}
$$

The zeroth-order solution in the new variables is given by

$$
\begin{aligned}
\mu_{0} & =\left\{\begin{array}{ll}
\frac{I_{0}\left(R_{0} r\right)}{R_{0} I_{1}\left(R_{0}\right)} & \text { for } \quad r<1 \\
\log r+\frac{I_{0}\left(R_{0}\right)}{R_{0} I_{1}\left(R_{0}\right)} & \text { for } \quad r>1
\end{array},\right. \\
u_{0}(r)=\frac{1}{I_{1}\left(R_{0}\right)} \int_{r}^{1} I_{1}\left(R_{0} \xi\right) \mathrm{d} \xi & \text { for } \quad r<1,
\end{aligned}
$$

where $\beta_{0}=1 / R_{0}$. Clearly

$$
\begin{aligned}
& \frac{\partial \mu_{0}}{\partial r}=\frac{I_{1}\left(R_{0} r\right)}{I_{1}\left(R_{0}\right)} \quad \text { for } r<1, \quad=\frac{1}{r} \quad \text { for } r>1, \\
& \frac{\partial u_{0}}{\partial r}=-\frac{I_{1}\left(R_{0} r\right)}{I_{1}\left(R_{0}\right)} \quad \text { for } r<1 .
\end{aligned}
$$

Substituting (5.2) and (5.9)-(5.12) into the system (5.4), (5.5), (5.8) we find that $\left(\mu_{n}, u_{n}, \lambda_{n}, \beta_{n}\right)$ satisfy the following system:

$$
\begin{aligned}
& \Delta \mu_{n}-\chi_{\{r<1\}}\left(R^{2} \mu_{n}+2 R \lambda_{n} \mu_{0}\right)-\frac{1}{R} \lambda_{n, \theta \theta} \frac{1}{r} \frac{\partial \mu_{0}}{\partial r}=F^{1 n}(r, \theta) \quad \text { in } \mathbb{R}^{2}, \\
& \Delta u_{n}+\left(R^{2} \mu_{n}+2 R \lambda_{n} \mu_{0}\right)-\frac{1}{R} \lambda_{n, \theta \theta} \frac{1}{r} \frac{\partial u_{0}}{\partial r}=F^{2 n}(r, \theta) \quad \text { in }\{r<1\},
\end{aligned}
$$


where $R=R_{0}=R_{0 l}$, with the boundary conditions

$$
\begin{aligned}
& {\left[\mu_{n}\right]=\left[\frac{\partial \mu_{n}}{\partial r}\right]=0 \quad \text { on } r=1,} \\
& u_{n}=0 \quad \text { on } r=1, \\
& \frac{\partial u_{n}}{\partial r}+\beta_{n} R+\frac{1}{R} \lambda_{n}=F^{3 n}(\theta) \quad \text { on } r=1 .
\end{aligned}
$$

The $F^{j n}$ depend only on the $\mu_{m}, u_{m}, \lambda_{m}, \beta_{m}$ for $m<n$.

By reversing the mapping

$$
r \rightarrow \frac{r}{R_{0}+f(\theta, \varepsilon)}
$$

and using the results of Section 4 we can deduce that the system (5.17)-(5.21) has a solution which is even in $\theta$.

In the next section we prove general lemmas which will enable us (in Section 7) to derive bounds for the system (5.17)-(5.21) that ensure convergence of the power series (5.9)-(5.12).

\section{Fundamental lemmas}

We introduce the norm

$$
\|N\|_{H_{r}^{2}\left(B_{1}\right)}=\left\||N|+\frac{1}{r}\left|\frac{\partial N}{\partial r}\right|+\left|\frac{\partial^{2} N}{\partial r^{2}}\right|+\frac{1}{r}\left|\frac{\partial^{2} N}{\partial r \partial \theta}\right|+\frac{1}{r^{2}}\left|\frac{\partial^{2} N}{\partial \theta^{2}}\right|\right\|_{L^{2}\left(B_{1}\right)}
$$

where $B_{1}$ is the unit ball in $\mathbb{R}^{2}$. This rather unusual norm is related to the form of the operators in (5.8), (5.9), and will be very useful. It is easily seen that

$$
\|N\|_{H^{2}\left(B_{1}\right)} \leqslant C\|N\|_{H_{r}^{2}\left(B_{1}\right)} .
$$

As proved in [5], if $N=0$ on $\partial B_{1}$ and

$$
\int_{0}^{2 \pi} N \cos \theta \mathrm{d} \theta=\int_{0}^{2 \pi} N \sin \theta \mathrm{d} \theta=0
$$

then also

$$
\|N\|_{H_{r}^{2}\left(B_{1}\right)} \leqslant C\|N\|_{H^{2}\left(B_{1}\right)} .
$$

The condition $N=0$ on $\partial B_{1}$ may actually be dropped by applying the last inequality to $N-N_{0}$ where $\Delta N_{0}=0$ in $B_{1}, N_{0}=N$ on $\partial B_{1}$, and then estimating $\left\|N_{0}\right\|_{H_{r}^{2}\left(B_{1}\right)}$ using the expansion of $N_{0}$ into a series $\sum_{m \neq \pm 1} a_{m} r^{m} \mathrm{e}^{\mathrm{i} m \theta}$.

Consider the system for $M=M(r, \theta), U=U(r, \theta), \Lambda=\Lambda(\theta)$ :

$$
\begin{aligned}
& \Delta M-\chi_{\{r<1\}}\left(R^{2} M+2 R \Lambda \mu_{0}\right)-\frac{1}{R} \Lambda_{\theta \theta} \frac{1}{r} \frac{\partial \mu_{0}}{\partial r}=F^{1}(r, \theta) \quad \text { in } \mathbb{R}^{2}, \\
& \Delta U+\left(R^{2} M+2 R \Lambda \mu_{0}\right)-\frac{1}{R} \Lambda_{\theta \theta} \frac{1}{r} \frac{\partial u_{0}}{\partial r}=F^{2}(r, \theta) \quad \text { in }\{r<1\},
\end{aligned}
$$


with the boundary conditions

$$
\begin{aligned}
& {[M]=\left[\frac{\partial M}{\partial r}\right]=0 \quad \text { on } r=1,} \\
& U=0 \quad \text { on } r=1, \\
& \frac{\partial U}{\partial r}+\beta R+\frac{1}{R} \Lambda=F^{3}(\theta) \quad \text { on } r=1,
\end{aligned}
$$

where $\beta$ is a given real number.

REMARK 6.1 (Relation to bifurcation theory.) As in general bifurcation theory, let us write

$$
\begin{aligned}
& u=u_{0}(r)+\varepsilon \tilde{u}, \\
& \mu=\mu_{0}(r)+\varepsilon \tilde{\mu}, \\
& f=R_{0}+\varepsilon \tilde{\lambda} \quad\left(\tilde{\lambda}=\cos l \theta+\varepsilon \tilde{\lambda}_{1}\right) \\
& \beta=\beta_{0}+\varepsilon \widetilde{\beta}
\end{aligned}
$$

and split the system (5.4)-(5.8) analogously to (1.14), (1.15). We write the analogue of (1.14) in the form

$$
\Pi(\widetilde{U}, \widetilde{\beta})=\widetilde{F}
$$

where $\widetilde{U}=(\tilde{u}, \tilde{\mu}, \tilde{\lambda}) ; \tilde{F}$ actually depends on $\widetilde{U}$. The implicit function theorem requires the invertibility of (6.8) in certain Banach spaces. As a step toward establishing this fact, we need to prove that, for any $\widetilde{F}$ in a suitable Banach space $B_{1}$ there is a unique properly normalized solution $\widetilde{U}$ of (6.8) in a Banach space $B_{2}$, and

$$
\|\widetilde{U}\|_{B_{2}} \leqslant C\|\widetilde{F}\|_{B_{1}}
$$

The Banach space $B_{1}$, in our case, turns out to be very complicated. It includes norms such as (6.12) below and corresponding norms of $\theta$-derivatives to all orders with appropriate weights. For clarity, we shall state (in this section) the estimate (6.9) just for the norm (6.12). The class of functions $\widetilde{F}$ is described in Lemma 6.4, and consists of a linear combination of several subspaces of functions, dealt with separately in Lemmas 6.1-6.3.

With Lemma 6.4 at hand we shall proceed in Section 7 to invert the problem (6.8) (for $\widetilde{F}$ which is a nonlinear function of $\widetilde{U}$ ). This inversion, however, is not straightforward. It requires a compatibility result (Lemma 7.1). It also requires a delicate examination of the coefficients of $\beta_{n}$ as they appear in the equations that determine $u_{n+1}, \mu_{n+1}, \lambda_{n+1}$.

The analysis of Section 7 is written in the form of inductive estimates on $u_{n}, \mu_{n}, f_{n}, \beta_{n-1}$ and all their $\theta$-derivatives. The end result could probably be formulated as a rather complicated analytic implicit function theorem (involving the aggregate $\left\{u_{n}, \mu_{n}, f_{n}, \beta_{n-1} ; n=1,2,3, \ldots\right\}$, but such a formulation seems less useful than the more direct approach of the inductive estimates. The final conclusion is that there exists an analytic branch of solutions in the transformed variables. The authors are not aware of any analytic implicit function theorem in Banach spaces which applies to the present problem. 
LEMma 6.1 Set $R=R_{0}=R_{0 l}$ and let $F^{j}$ be even functions of $\theta$ such that $F^{1} \in L^{2}\left(\mathbb{R}^{2}\right), F^{2} \in$ $L^{2}\left(B_{1}\right), F^{3} \in H^{1 / 2}\left(\partial B_{1}\right)$,

$$
\begin{array}{r}
\int_{0}^{2 \pi} F^{1}(r, \theta) \cos m \theta \mathrm{d} \theta \equiv 0, \quad \int_{0}^{2 \pi} F^{2}(r, \theta) \cos m \theta \mathrm{d} \theta \equiv 0, \\
\int_{0}^{2 \pi} F^{3}(\theta) \cos m \theta \mathrm{d} \theta=0 \quad \text { for } m=1, l,
\end{array}
$$

and, for $r>1$,

$$
\begin{array}{r}
F^{1}(r, \theta)=\frac{1}{r^{2}} F^{10}(\theta)+\frac{1}{r^{2}} F^{11}(r, \theta), \\
\int_{0}^{2 \pi} F^{10}(\theta) \mathrm{d} \theta=0, \quad\left\|F^{11}\right\|_{L^{2}\left(\mathbb{R}^{2} \backslash B_{1}\right)}<\infty .
\end{array}
$$

Set

$$
\left\||F \||=\left(\left\|F^{1}\right\|_{L^{2}\left(B_{1}\right)}+\left\|F^{10}\right\|_{L^{2}\left(\partial B_{1}\right)}+\left\|F^{11}\right\|_{L^{2}\left(\mathbb{R}^{2} \backslash B_{1}\right)}+\left\|F^{2}\right\|_{L^{2}\left(B_{1}\right)}+\left\|F^{3}\right\|_{H^{1 / 2}\left(\partial B_{1}\right)}\right) .\right.
$$

Then there exists a unique solution $(M, U, \Lambda)$ of (6.3)-(6.7), even in $\theta$, such that

$$
\begin{array}{r}
\int_{0}^{2 \pi} M(r, \theta) \cos m \theta \mathrm{d} \theta \equiv 0, \quad \int_{0}^{2 \pi} U(r, \theta) \cos m \theta \mathrm{d} \theta \equiv 0, \\
\int_{0}^{2 \pi} \Lambda(\theta) \cos m \theta \mathrm{d} \theta=0 \quad \text { for } m=1, l,
\end{array}
$$

and the following estimates hold:

$$
\begin{aligned}
& \left\|\left(\left|M-M_{0}\right|+\left|\frac{1}{r} \frac{\partial\left(M-M_{0}\right)}{\partial r}\right|+\left|\frac{1}{r} \frac{\partial M}{\partial \theta}\right|+\left|\frac{\partial^{2}\left(M-M_{0}\right)}{\partial r^{2}}\right|+\left|\frac{1}{r} \frac{\partial^{2} M}{\partial r \partial \theta}\right|\right)\right\|_{L^{2}\left(B_{1}\right)} \\
& +\left\|M+U-C_{20}\right\|_{H^{2}\left(B_{1}\right)}+\left\|\Lambda+\beta R^{2}\right\|_{H^{3 / 2}\left(\partial B_{1}\right)}+\left|C_{20}-\beta R\left(\frac{I_{0}^{2}(R)}{I_{1}^{2}(R)}-1\right)\right| \leqslant C\|F\|,
\end{aligned}
$$

and

$$
\left\|r^{2}\left(\left|\frac{1}{r} \frac{\partial M}{\partial r}\right|+\left|\frac{\partial^{2} M}{\partial r^{2}}\right|+\left|\frac{1}{r} \frac{\partial^{2} M}{\partial r \partial \theta}\right|\right)\right\|_{L^{2}\left(\mathbb{R}^{2} \backslash B_{1}\right)} \leqslant C\|\mid F\|,
$$

where $C_{20}$ is a constant,

$$
M_{0}(r)=M_{0}^{(1)}(r)+M_{0}^{(3)}(r) \quad \text { for } r<1,
$$

and

$$
\begin{aligned}
M_{0}^{(3)}(r) & =-\frac{\Lambda_{0}}{R}\left[\frac{I_{0}(R r)}{I_{0}(R)}-\frac{r I_{1}(R r)}{I_{1}(R}\right], \\
M_{0}^{(1)}(r) & =C_{20} \frac{I_{0}(R r)}{I_{0}(R)}, \\
\Lambda_{0} & =\frac{1}{2 \pi} \int_{0}^{2 \pi} \Lambda(\theta) \mathrm{d} \theta,
\end{aligned}
$$

and $C$ is a constant independent of $\beta$. 
We recall that the norms $H^{k}$ for $k$ which is not as integer can be defined either by the Fourier transform, or by interpolation [1].

It is worth pointing out that the first term on the left-hand side of (6.14) is not the $H_{r}^{2}$ norm of $M-M_{0}$, as the pure second-order $\theta$ derivative is missing. Note, also, that on the left-hand side of (6.15) there is a weight $r^{2}$ inside the $L^{2}$-norm; this is enabled by the factor $1 / r^{2}$ in (6.11).

REMARK 6.2 It may look rather surprising that $\Lambda+\beta R^{2}$ has one more derivative than $F^{3}$, since this is not apparent from the boundary condition (6.7). The reason for this gain of one derivative is roughly the following: equation (6.3) suggests that the regularity of $M$ is the same as the regularity of $\Lambda$. Since $M+U$ satisfies a 'nice' Poisson equation, $\partial U / \partial r$ is 'likely' to have the same regularity as $\partial M / \partial r$ at $r=1$, i.e. as $\Lambda_{\theta}$. Thus (6.7) is in some sense a pseudo-differential operator of the first order for $\Lambda$, and thus should have one more derivative than $F^{3}$.

Proof. By the assumptions on the $F^{j}$ we can write

$$
\begin{aligned}
& F^{j}(r, \theta)=\sum_{m \neq 1, m \neq l} F_{m}^{j}(r) \cos m \theta, \quad j=1,2, \\
& F^{3}(\theta)=\sum_{m \neq 1, m \neq l} F_{m}^{3} \cos m \theta .
\end{aligned}
$$

Furthermore, for $r>1$,

$$
F_{m}^{1}(r)=\frac{1}{r^{2}}\left(F_{m}^{10}+F_{m}^{11}(r)\right)
$$

where

$$
\begin{aligned}
& F_{m}^{10}=\frac{1}{\pi} \int_{0}^{2 \pi} F^{10}(\theta) \cos m \theta \mathrm{d} \theta \quad \text { if } m \geqslant 2, \quad F_{0}^{10}=0 \\
& F_{m}^{11}(r)=\frac{1}{\pi} \int_{0}^{2 \pi} F^{11}(r, \theta) \cos m \theta \mathrm{d} \theta \quad \text { if } \quad m \geqslant 2, \\
& F_{0}^{11}(r)=\frac{1}{2 \pi} \int_{0}^{2 \pi} F^{11}(r, \theta) \mathrm{d} \theta .
\end{aligned}
$$

In view of (6.13) the solution $\Lambda, M, U$ must have the form

$$
\begin{aligned}
\Lambda & =\sum_{m \neq 1, m \neq l} \Lambda_{m} \cos m \theta, \\
M & =\sum_{m \neq 1, m \neq l} M_{m}(r) \cos m \theta, \\
U & =\sum_{m \neq 1, m \neq l} U_{m}(r) \cos m \theta .
\end{aligned}
$$

Then $\left(M_{m}, U_{m}, \Lambda_{m}\right)$ satisfy

$$
\begin{aligned}
& M_{m}^{\prime \prime}+\frac{1}{r} M_{m}^{\prime}-\frac{m^{2}}{r^{2}} M_{m}-\chi_{\{r<1\}}\left(R^{2} M_{m}+2 R \Lambda_{m} \mu_{0}\right)+\frac{1}{R} m^{2} \Lambda_{m} \frac{1}{r} \frac{\partial \mu_{0}}{\partial r}=F_{m}^{1} \quad \text { in } \mathbb{R}^{2}, \\
& U_{m}^{\prime \prime}+\frac{1}{r} U_{m}^{\prime}-\frac{m^{2}}{r^{2}} U_{m}+\left(R^{2} M_{m}+2 R \Lambda_{m} \mu_{0}\right)+\frac{1}{R} m^{2} \Lambda_{m} \frac{1}{r} \frac{\partial u_{0}}{\partial r}=F_{m}^{2} \quad \text { for } \quad r<1,
\end{aligned}
$$


with the boundary conditions

$$
\begin{aligned}
& {\left[M_{m}\right]=\left[\frac{\partial M_{m}}{\partial r}\right]=0 \quad \text { on } \quad r=1,} \\
& U_{m}=0 \quad \text { on } \quad r=1, \\
& \frac{\partial U_{m}}{\partial r}+\frac{1}{R} \Lambda_{m}+\beta R \delta_{m 0}=F_{m}^{3} \quad \text { on } \quad r=1 .
\end{aligned}
$$

The general solution of (6.23) can be written in the form

$$
M_{m}=M_{m}^{(1)}+M_{m}^{(2)}+M_{m}^{(3)},
$$

where $M_{m}^{(1)}$ corresponds to the general homogeneous solution, namely

$$
M_{m}^{(1)}= \begin{cases}C_{1 m} I_{m}(R r) & \text { for } \quad r<1 \\ C_{2 m} r^{-m} & \text { for } \quad r>1 .\end{cases}
$$

$M_{m}^{(2)}$ corresponds to an inhomogeneous solution involving $F_{m}^{1}(r)$, and $M_{m}^{(3)}$ corresponds to an inhomogeneous solution involving $\Lambda_{m}$. Writing $M_{m}^{(2)}=g_{m}(r) I_{m}(R r)$ for $r<1$ and $M_{m}^{(2)}=$ $g_{m}(r) r^{-m}$ for $r>1$, we derive a first-order ODE for $g_{m}^{\prime}(r)$ which can be readily solved by integration. This leads to

$$
M_{m}^{(2)}= \begin{cases}-I_{m}(R r) \int_{r}^{1} \frac{\mathrm{d} s}{s I_{m}^{2}(R s)} \int_{0}^{s} \tau I_{m}(R \tau) F_{m}^{1}(\tau) \mathrm{d} \tau & \text { for } \quad r<1 \\ -\frac{1}{r^{m}} \int_{1}^{r} s^{2 m-1} \mathrm{~d} s \int_{s}^{\infty} \frac{F_{m}^{1}(\tau)}{\tau^{m-1}} \mathrm{~d} \tau & \text { for } \quad r>1\end{cases}
$$

Finally, recalling (5.13) and (5.15), a solution $M_{m}^{(3)}$ is obtained by replacing, in (6.30), $F_{m}^{1}(r)$ by

$$
\left\{\begin{array}{l}
\frac{\Lambda_{m}}{I_{1}(R)}\left\{2 I_{0}(R r)-\frac{m^{2}}{R r} I_{1}(R r)\right\} \quad \text { for } \quad r<1, \\
-\frac{m^{2}}{R} \frac{1}{r^{2}} \Lambda_{m} \quad \text { for } \quad r>1 .
\end{array}\right.
$$

This yields, for $r>1$,

$$
\begin{aligned}
& M_{m}^{(3)}(r)=\frac{\Lambda_{m}}{r^{m}} \int_{1}^{r} s^{2 m-1} \mathrm{~d} s \int_{s}^{\infty} \frac{m^{2}}{R \tau^{m+1}} \mathrm{~d} \tau=\frac{\Lambda_{m}}{R}\left(1-\frac{1}{r^{m}}\right) \quad \text { for } \quad m \geqslant 2, \\
& M_{0}^{(3)}(r)=0 .
\end{aligned}
$$

To compute $M_{m}^{(3)}(r)$ for $r<1$, we use Theorem A.3:

$$
\begin{aligned}
M_{m}^{(3)}(r)= & \frac{\Lambda_{m}}{R} \frac{I_{m}(R r)}{I_{1}(R)} \int_{r}^{1} \frac{\mathrm{d} s}{s I_{m}^{2}(R s)} \int_{0}^{R s} \frac{1}{R} I_{m}(\tau)\left\{m^{2} I_{1}(\tau)-2 \tau I_{0}(\tau)\right\} \mathrm{d} \tau \\
= & \frac{\Lambda_{m}}{R} \frac{I_{m}(R r)}{I_{1}(R)} \int_{r}^{1} \frac{R s}{I_{m}^{2}(R s)}\left\{\frac{m-2}{R s} I_{1}(R s) I_{m}(R s)\right. \\
& \left.-\left[I_{2}(R s) I_{m}(R s)-I_{1}(R s) I_{m+1}(R s)\right]\right\} \mathrm{d} s,
\end{aligned}
$$


so that

$$
M_{m}^{(3)}(r)=\frac{\Lambda_{m}}{R} \frac{I_{m}(R r)}{I_{1}(R)} B_{m}(r) \quad \text { for } \quad m \geqslant 2,
$$

where, in view of the relations $\frac{I_{2}(r)}{I_{1}(r)}=\frac{I_{0}(r)}{I_{1}(r)}-\frac{2}{r}$,

$$
B_{m}(r)=\int_{r}^{1} \frac{I_{1}(R s)}{I_{m}(R s)}\left\{m-(R s)\left[\frac{I_{0}(R s)}{I_{1}(R s)}-\frac{I_{m+1}(R s)}{I_{m}(R s)}\right]\right\} \mathrm{d} s \quad \text { if } \quad m \geqslant 2 .
$$

The same formula is valid for $m=0$ with

$$
\begin{aligned}
B_{0}(r) & =-\int_{r}^{1} \frac{\mathrm{d} s}{s I_{0}^{2}(R s)} \int_{0}^{R s} \frac{2}{R} \tau I_{0}^{2}(\tau) \mathrm{d} \tau=-\left.\int_{r}^{1} \frac{\mathrm{d} s}{R s I_{0}^{2}(R s)}(\tau)^{2}\left[I_{0}^{2}(\tau)-I_{1}^{2}(\tau)\right]\right|_{0} ^{R s} \\
& =-\int_{r}^{1}(R s)\left[1-\frac{I_{1}^{2}(R s)}{I_{0}^{2}(R s)}\right] \mathrm{d} s=-\left.s \frac{I_{1}(R s)}{I_{0}(R s)}\right|_{r} ^{1},
\end{aligned}
$$

or

$$
B_{0}(r)=-\frac{I_{1}(R)}{I_{0}(R)}+r \frac{I_{1}(R r)}{I_{0}(R r)} .
$$

The second and third equalities above can be verified by differentiating the right-hand sides and using the relations $\left(x I_{1}(x)\right)^{\prime}=x I_{0}(x), I_{0}^{\prime}(x)=I_{1}(x)$. In particular, we conclude that (6.17) is valid.

We next add equations (6.23) and (6.24) to get

$$
\left(U_{m}+M_{m}\right)^{\prime \prime}+\frac{1}{r}\left(U_{m}+M_{m}\right)^{\prime}-\frac{m^{2}}{r^{2}}\left(U_{m}+M_{m}\right)=F_{m}^{1}+F_{m}^{2},
$$

which leads to

$$
U_{m}=-M_{m}+C_{3 m} r^{m}-r^{m} \int_{r}^{1} \frac{\mathrm{d} s}{s^{2 m+1}} \int_{0}^{s} \tau^{m+1}\left[F_{m}^{1}(\tau)+F_{m}^{2}(\tau)\right] \mathrm{d} \tau .
$$

We divide the rest of the proof of the lemma into six steps.

Step 1. Solving the system for $\left(C_{1 m}, C_{2 m}, C_{3 m}, \Lambda_{m} / R\right)$.

Substituting the expressions for $M_{m}, U_{m}$ from (6.28), (6.35) into the boundary conditions (6.25)-(6.27), we find, after using the relation (A.5), that

$$
\begin{aligned}
& C_{1 m} I_{m}(R)=C_{2 m}, \\
& C_{1 m} R I_{m}^{\prime}(R)+\frac{1}{I_{m}(R)} \int_{0}^{1} \tau I_{m}(R \tau) F_{m}^{1}(\tau) \mathrm{d} \tau+\frac{\Lambda_{m}}{R}\left[m+R\left(\frac{I_{0}(R)}{I_{1}(R)}-\frac{I_{m-1}(R)}{I_{m}(R)}\right)\right] \\
& \quad=-m C_{2 m}+\frac{\Lambda_{m}}{R} m-\int_{1}^{\infty} \frac{F_{m}^{1}(\tau)}{\tau^{m-1}} \mathrm{~d} \tau, \\
& C_{2 m}=C_{3 m}, \\
& m C_{3 m}+\int_{0}^{1} \tau^{m+1}\left[F_{m}^{1}(\tau)+F_{m}^{2}(\tau)\right] \mathrm{d} \tau+\frac{\Lambda_{m}}{R}+\delta_{m 0} \beta R \\
& \quad=-m C_{2 m}+\frac{\Lambda_{m}}{R} m-\int_{1}^{\infty} \frac{F_{m}^{1}(\tau)}{\tau^{m-1}} \mathrm{~d} \tau+F_{m}^{3} .
\end{aligned}
$$


Using (6.36) and (6.38) to eliminate $C_{1 m}$ and $C_{3 m}$, we obtain a system of equations for $C_{2 m}$ and $\Lambda_{m} / R$ which, after using (A.3), takes the form:

$$
\begin{aligned}
C_{2 m} & \frac{R I_{m-1}(R)}{I_{m}(R)}+\frac{\Lambda_{m}}{R} R\left(\frac{I_{0}(R)}{I_{1}(R)}-\frac{I_{m-1}(R)}{I_{m}(R)}\right) \\
= & -\int_{1}^{\infty} \frac{F_{m}^{1}(\tau)}{\tau^{m-1}} \mathrm{~d} \tau-\frac{1}{I_{m}(R)} \int_{0}^{1} \tau I_{m}(R \tau) F_{m}^{1}(\tau) \mathrm{d} \tau, \\
C_{2 m} 2 m & +\frac{\Lambda_{m}}{R}(1-m)=F_{m}^{3}-\delta_{m 0} \beta R \\
& -\int_{0}^{1} \tau^{m+1}\left[F_{m}^{1}(\tau)+F_{m}^{2}(\tau)\right] \mathrm{d} \tau-\int_{1}^{\infty} \frac{F_{m}^{1}(\tau)}{\tau^{m-1}} \mathrm{~d} \tau .
\end{aligned}
$$

Since $R=R_{0 l}$, the determinant of the coefficients matrix of (6.40), (6.41) is

$$
R\left[(m+1) \frac{I_{m-1}(R)}{I_{m}(R)}-2 m \frac{I_{0}(R)}{I_{1}(R)}\right] \neq 0 \quad \text { by Theorem A.2, }
$$

and, consequently, the system (6.23)-(6.27) has a unique solution $\left(C_{1 m}, C_{2 m}, C_{3 m}, \Lambda_{m} / R\right)$.

REMARK 6.3 If $m=0$ then the quantity

$$
\frac{\Lambda_{m}}{R}\left[m+R\left(\frac{I_{0}(R)}{I_{1}(R)}-\frac{I_{m-1}(R)}{I_{m}(R)}\right)\right]
$$

in (6.37) (which is the expression for $\partial M_{m}^{(3)} / \partial r$ at $r=R$ ) should be replaced by the expression $B_{0}^{\prime}(1) I_{0}(R) / I_{1}(R)$, which is equal to

$$
\frac{\Lambda_{0}}{R} R\left(\frac{I_{0}(R)}{I_{1}(R)}-\frac{I_{1}(R)}{I_{0}(R)}\right)
$$

This implies that, in (6.40), if $m=0$ then $I_{m-1}(R) / I_{m}(R)$ should be replaced by $I_{1}(R) / I_{0}(R)$.

Step 2. Estimating $\|\Lambda+\beta R\|_{H^{3 / 2}\left(\partial B_{1}\right)}$

By (A.2), $I_{m}(R) / I_{m-1}(R) \leqslant R / 2 m \rightarrow 0$ as $m \rightarrow \infty$. Hence by (A.5),

$$
\frac{I_{m-1}(R)}{I_{m}(R)}=\frac{I_{m+1}(R)}{I_{m}(R)}+\frac{2 m}{R} \sim \frac{2 m}{R} \quad \text { for large } m,
$$

so that $T_{m} \sim 2 m^{2}$ for $m$ large. It is also clear from (A.4) that $R I_{m}^{\prime}(R) / I_{m}(R) \sim m$, and from (A.6) that

$$
\frac{I_{m}(R \tau)}{I_{m}(R)} \sim \tau^{m} \quad \text { uniformly in } \tau, \quad 0<\tau<1 .
$$


Using these relations in (6.40), (6.41), we find that for $m \geqslant 2, m \neq l$,

$$
\begin{aligned}
m\left(\left|\frac{\Lambda_{m}}{R}\right|+\left|C_{2 m}\right|\right) \leqslant & C\left\{\left|\int_{1}^{\infty} \frac{F_{m}^{1}(\tau)}{\tau^{m}} \tau \mathrm{d} \tau\right|+\left|\int_{0}^{1} \frac{I_{m}(R \tau)}{I_{m}(R)} F_{m}^{1}(\tau) \tau \mathrm{d} \tau\right|\right. \\
& \left.+\left|F_{m}^{3}\right|+\left|\int_{0}^{1} \tau^{m}\left(F_{m}^{1}(\tau)+F_{m}^{2}(\tau)\right) \tau \mathrm{d} \tau\right|\right\} \\
\leqslant & C\left(\int_{0}^{\infty}\left[F_{m}^{1}(\tau)\right]^{2} \tau \mathrm{d} \tau\right)^{1 / 2}\left(\int_{0}^{1} \tau^{2 m+1} \mathrm{~d} \tau+\int_{1}^{\infty} \frac{\mathrm{d} \tau}{\tau^{2 m-1}}\right)^{1 / 2} \\
& +C\left|F_{m}^{3}\right|+C\left(\int_{0}^{1}\left[F_{m}^{2}(\tau)\right]^{2} \tau \mathrm{d} \tau\right)^{1 / 2}\left(\int_{0}^{1} \tau^{2 m+1} \mathrm{~d} \tau\right)^{1 / 2} \\
\leqslant & \frac{C}{m^{1 / 2}}\left(\left\|F_{m}^{1}\right\|_{L^{2}\left(\mathbb{R}^{2}\right)}+\left\|F_{m}^{2}\right\|_{L^{2}\left(B_{1}\right)}\right)+C\left|F_{m}^{3}\right| .
\end{aligned}
$$

Consider now the case $m=0$. By (6.19),

$$
\left|\int_{1}^{\infty} \tau F_{0}^{1}(\tau) \mathrm{d} \tau\right|=\left|\frac{1}{2 \pi} \int_{0}^{2 \pi} \int_{1}^{\infty} \frac{F^{11}(\tau, \theta)}{\tau} \mathrm{d} \tau \mathrm{d} \theta\right| \leqslant C\left\|F^{11}\right\|_{L^{2}\left(\mathbb{R}^{2} \backslash B_{1}\right)} .
$$

Recall also that $M_{0}^{(3)}$ is computed from (6.20), which means that in (6.40) we replace the quantity $I_{m-1}(R) / I_{m}(R)$ by $I_{1}(R) / I_{0}(R)$ (see Remark 6.3). Solving (6.41) for $\frac{\Lambda_{0}}{R}+\beta$ and then substituting this into (6.40) and observing that

$$
C_{20} \frac{R I_{1}}{I_{0}}-\beta R^{2}\left(\frac{I_{0}}{I_{1}}-\frac{I_{1}}{I_{0}}\right)=\frac{R I_{1}}{I_{0}}\left[C_{20}-\beta R\left(\frac{I_{0}^{2}}{I_{1}^{2}}-1\right)\right]
$$

we get

$$
\left|\frac{\Lambda_{0}}{R}+\beta R\right|+\left|C_{20}-\beta R\left(\frac{I_{0}^{2}(R)}{I_{1}^{2}(R)}-1\right)\right| \leqslant C\left(\left\|F_{0}^{1}\right\|_{L^{2}\left(B_{1}\right)}+\left\|F^{11}\right\|_{L^{2}\left(\mathbb{R}^{2} \backslash B_{1}\right)}+\left\|F_{0}^{2}\right\|_{L^{2}\left(B_{1}\right)}+\left|F_{0}^{3}\right|\right) .
$$

The estimates (6.43) imply that

$$
\sum_{m \neq 1} m^{3}\left|\Lambda_{m}\right|^{2} \leqslant C\left\{\sum_{m \geqslant 2}\left(\left\|F_{m}^{1}\right\|_{L^{2}\left(\mathbb{R}^{2}\right)}^{2}+\left\|F_{m}^{2}\right\|_{L^{2}\left(B_{1}\right)}^{2}\right)+\sum_{m \geqslant 2} m\left|F_{m}^{3}\right|^{2}\right\}
$$

and, together with (6.44),

$$
\begin{aligned}
& \left\|\Lambda-\Lambda_{0}\right\|_{H^{3 / 2}\left(\partial B_{1}\right)} \leqslant C\left(\left\|F^{1}\right\|_{L^{2}\left(\mathbb{R}^{2}\right)}+\left\|F^{2}\right\|_{L^{2}\left(B_{1}\right)}+\left\|F^{3}\right\|_{H^{1 / 2}\left(\partial B_{1}\right)}\right), \\
& \left|\frac{\Lambda_{0}}{R}+\beta R\right|+\left|C_{20}-\beta R\left(\frac{I_{0}^{2}(R)}{I_{1}^{2}(R)}-1\right)\right| \\
& \quad \leqslant C\left(\left\|F^{1}\right\|_{L^{2}\left(B_{1}\right)}+\left\|F^{11}\right\|_{L^{2}\left(\mathbb{R}^{2} \backslash B_{1}\right)}+\left\|F^{2}\right\|_{L^{2}\left(B_{1}\right)}+\left\|F^{3}\right\|_{H^{1 / 2}\left(\partial B_{1}\right)}\right) .
\end{aligned}
$$

Step 3. Estimating $M^{(1)}$. 
For the region $r>1$, if $m \geqslant 2$,

$$
\begin{aligned}
& m^{2} \int_{1}^{\infty}\left[r \frac{\partial}{\partial r} M_{m}^{(1)}(r)\right]^{2} r \mathrm{~d} r+\int_{1}^{\infty}\left[r^{2} \frac{\partial^{2}}{\partial r^{2}} M_{m}^{(1)}(r)\right]^{2} r \mathrm{~d} r \\
& \quad \leqslant C m^{4}\left|C_{2 m}\right|^{2} \int_{1}^{\infty} r^{-2 m} r \mathrm{~d} r \leqslant C m\left|F_{m}^{3}\right|^{2}+C\left(\left\|F_{m}^{1}\right\|_{L^{2}\left(\mathbb{R}^{2}\right)}^{2}+\left\|F_{m}^{2}\right\|_{L^{2}\left(B_{1}\right)}^{2}\right),
\end{aligned}
$$

which implies the bound

$$
\begin{aligned}
& \left\|r M_{r}^{(1)}\right\|_{L^{2}\left(\mathbb{R}^{2} \backslash B_{1}\right)}+\left\|r M_{r \theta}^{(1)}\right\|_{L^{2}\left(\mathbb{R}^{2} \backslash B_{1}\right)}+\left\|r^{2} M_{r r}^{(1)}\right\|_{L^{2}\left(\mathbb{R}^{2} \backslash B_{1}\right)} \\
& \leqslant C\left(\left\|F^{1}\right\|_{L^{2}\left(\mathbb{R}^{2}\right)}+\left\|F^{2}\right\|_{L^{2}\left(B_{1}\right)}\right)+C\left\|F^{3}\right\|_{H^{1 / 2}\left(\partial B_{1}\right)} .
\end{aligned}
$$

Next we consider a bound on $M^{(1)}$ for $r<1$. Since $C_{1 m}=C_{2 m} / I_{m}(R)$, we have, by (6.29) and (6.42),

$$
\begin{aligned}
& m^{4} \int_{0}^{1} \frac{1}{r^{4}}\left[M_{m}^{(1)}(r)\right]^{2} r \mathrm{~d} r \leqslant m^{4}\left|C_{2 m}\right|^{2} \int_{0}^{1} \frac{1}{r^{4}}\left(\frac{I_{m}(R r)}{I_{m}(R)}\right)^{2} r \mathrm{~d} r \\
& \quad \leqslant C m^{4}\left|C_{2 m}\right|^{2} \int_{0}^{1} r^{2 m-4} r \mathrm{~d} r \leqslant C m\left|F_{m}^{3}\right|^{2}+C\left(\left\|F_{m}^{1}\right\|_{L^{2}\left(\mathbb{R}^{2}\right)}^{2}+\left\|F_{m}^{2}\right\|_{L^{2}\left(B_{1}\right)}^{2}\right),
\end{aligned}
$$

if $m \geqslant 2$, where the last inequality follows by (6.43). Also, by (A.3)-(A.5) we easily verify that

$$
\left|\frac{\partial}{\partial r} I_{m}(r)\right| \leqslant C m \frac{I_{m}(r)}{r}, \quad\left|\frac{\partial^{2}}{\partial r^{2}} I_{m}(r)\right| \leqslant C m^{2} \frac{I_{m}(r)}{r^{2}},
$$

so that

$$
\begin{aligned}
& m^{2} \int_{0}^{1} \frac{1}{r^{2}}\left[\frac{\partial}{\partial r} M_{m}^{(1)}(r)\right]^{2} r \mathrm{~d} r+\int_{0}^{1}\left[\frac{\partial^{2}}{\partial r^{2}} M_{m}^{(1)}(r)\right]^{2} r \mathrm{~d} r \\
& \leqslant C m^{4}\left|C_{2 m}\right|^{2} \int_{0}^{1}\left(\frac{I_{m}(R r)}{I_{m}(R)}\right)^{2} r \mathrm{~d} r \leqslant C m\left|F_{m}^{3}\right|^{2}+C\left(\left\|F_{m}^{1}\right\|_{L^{2}\left(\mathbb{R}^{2}\right)}^{2}+\left\|F_{m}^{2}\right\|_{L^{2}\left(B_{1}\right)}^{2}\right),
\end{aligned}
$$

if $m \geqslant 2$. Thus, altogether,

$$
\begin{gathered}
\left\|M^{(1)}-M_{0}^{(1)}(r)\right\|_{H_{r}^{2}\left(B_{1}\right)} \leqslant C\left(\left\|F^{1}\right\|_{L^{2}\left(B_{1}\right)}+\left\|F^{10}\right\|_{L^{2}\left(\partial B_{1}\right)}+\left\|F^{11}\right\|_{L^{2}\left(\mathbb{R}^{2} \backslash B_{1}\right)}\right. \\
\left.+\left\|F^{2}\right\|_{L^{2}\left(B_{1}\right)}+\left\|F^{3}\right\|_{H^{1 / 2}\left(\partial B_{1}\right)}\right) .
\end{gathered}
$$

Step 4. Estimating $M^{(2)}$.

For $r<1, M^{(2)}=\sum M_{m}^{(2)}(r) \cos m \theta$ satisfies

$$
\Delta M^{(2)}-R^{2} M^{(2)}=F^{1} \quad \text { in } B_{1}, \quad M^{(2)}=0 \quad \text { on } \partial B_{1} .
$$

By elliptic $L^{2}$ estimates, $\left\|M^{(2)}\right\|_{H^{2}\left(B_{1}\right)} \leqslant C\left\|F^{1}\right\|_{L^{2}\left(B_{1}\right)}$ so that, by (6.1)

$$
\left\|M^{(2)}\right\|_{H_{r}^{2}\left(B_{1}\right)} \leqslant C\left\|F^{1}\right\|_{L^{2}\left(B_{1}\right)} .
$$


To estimate $M^{(2)}(r, \theta)$ in $r>1$, we use (6.30) and (6.19). We can then write $M^{(2)}=M^{(20)}+$ $M^{(21)}$

$$
\begin{aligned}
& M^{(20)}(r, \theta)=\sum M_{m}^{(20)}(r) \cos m \theta, \\
& M^{(21)}(r, \theta)=\sum M_{m}^{(21)}(r) \cos m \theta,
\end{aligned}
$$

where

$$
\begin{aligned}
& M_{m}^{(20)}(r)=-\frac{1}{r^{m}} \int_{1}^{r} s^{2 m-1} \int_{s}^{\infty} \frac{F^{10}}{\tau^{m+1}} \mathrm{~d} \tau=-\frac{F^{10}}{m^{2}}\left(1-\frac{1}{r^{m}}\right) \quad \text { if } \quad m \geqslant 2, \\
& M_{0}^{(20)}=0, \\
& M_{m}^{(21)}(r)=-\frac{1}{r^{m}} \int_{1}^{r} s^{2 m-1} \int_{s}^{\infty} \frac{F_{m}^{11}(\tau)}{\tau^{m+1}} \mathrm{~d} \tau .
\end{aligned}
$$

Hence

$$
m^{2} \int_{1}^{\infty}\left(r \frac{\partial}{\partial r} M_{m}^{(20)}(r)\right)^{2} r \mathrm{~d} r+\int_{1}^{\infty}\left(r^{2} \frac{\partial^{2}}{\partial r^{2}} M_{m}^{(20)}(r)\right)^{2} r \mathrm{~d} r \leqslant \frac{C}{m}\left|F_{m}^{10}\right|^{2} \leqslant C\left|F_{m}^{10}\right|^{2},
$$

and this allows us to deduce that

$$
\left\|r M_{r}^{(20)}\right\|_{L^{2}\left(\mathbb{R}^{2} \backslash B_{1}\right)}+\left\|r M_{r \theta}^{(20)}\right\|_{L^{2}\left(\mathbb{R}^{2} \backslash B_{1}\right)}+\left\|r^{2} M_{r r}^{(20)}\right\|_{L^{2}\left(\mathbb{R}^{2} \backslash B_{1}\right)} \leqslant C\left\|F^{10}\right\|_{L^{2}\left(\partial B_{1}\right)} .
$$

Next, by direct computation ( $m=0$ is included in the computation)

$$
\begin{aligned}
\frac{\partial}{\partial r} M_{m}^{(21)}(r) & =\frac{m}{r^{m+1}} \int_{1}^{r} s^{2 m-1} \int_{s}^{\infty} \frac{F_{m}^{11}(\tau)}{\tau^{m+1}} \mathrm{~d} \tau-r^{m-1} \int_{r}^{\infty} \frac{F_{m}^{11}(\tau)}{\tau^{m+1}} \mathrm{~d} \tau, \\
\frac{\partial^{2}}{\partial r^{2}} M_{m}^{(21)}(r) & =-\frac{m(m+1)}{r^{m+2}} \int_{1}^{r} s^{2 m-1} \int_{s}^{\infty} \frac{F_{m}^{11}(\tau)}{\tau^{m+1}} \mathrm{~d} \tau+r^{m-2} \int_{r}^{\infty} \frac{F_{m}^{11}(\tau)}{\tau^{m+1}} \mathrm{~d} \tau+\frac{F_{m}^{11}(r)}{r^{2}} .
\end{aligned}
$$

Therefore, for all $m \geqslant 0$,

$$
\begin{aligned}
& \left|(m+1) r \frac{\partial}{\partial r} M_{m}^{(21)}(r)\right|+\left|r^{2} \frac{\partial^{2}}{\partial r^{2}} M_{m}^{(21)}(r)\right| \\
& \leqslant C\left\{\frac{m(m+1)}{r^{m}} \int_{1}^{r} s^{2 m-1} \int_{s}^{\infty} \frac{\left|F_{m}^{11}(\tau)\right|}{\tau^{m+1}} \mathrm{~d} \tau+(m+1) r^{m} \int_{r}^{\infty} \frac{\left|F_{m}^{11}(\tau)\right|}{\tau^{m+1}} \mathrm{~d} \tau+\left|F_{m}^{11}(r)\right|\right\} .
\end{aligned}
$$

We proceed to estimate the terms on the right-hand side by the method used in [5: Lemma 8.1]. We can write

$$
\begin{aligned}
& \frac{m(m+1)}{r^{m}} \int_{1}^{r} s^{2 m-1} \int_{s}^{\infty} \frac{\left|F_{m}^{11}(\tau)\right|}{\tau^{m+1}} \mathrm{~d} \tau \\
& \quad=\frac{m(m+1)}{r^{m}} \int_{1}^{\infty} \frac{\left|F_{m}^{11}(\tau)\right|}{\tau^{m+1}} \int_{1}^{\min (r, \tau)} s^{2 m-1} \mathrm{~d} s \mathrm{~d} \tau \\
& \quad \leqslant \frac{m(m+1)}{r^{m}}\left\{\int_{1}^{r} \frac{\left|F_{m}^{11}(\tau)\right|}{\tau^{m+1}} \frac{\tau^{2 m}}{m} \mathrm{~d} \tau+\int_{r}^{\infty} \frac{\left|F_{m}^{11}(\tau)\right|}{\tau^{m+1}} \frac{r^{2 m}}{m} \mathrm{~d} \tau\right\} \\
& \quad \leqslant \frac{(m+1)}{r^{m}} \int_{1}^{r}\left|F_{m}^{11}(\tau)\right| \tau^{m-1} \mathrm{~d} \tau+(m+1) r^{m} \int_{r}^{\infty} \frac{\left|F_{m}^{11}(\tau)\right|}{\tau^{m+1}} \mathrm{~d} \tau
\end{aligned}
$$


(notice that the left-hand side of the last inequality is 0 when $m=0$ ) so that, for all $m \geqslant 0$,

$$
\begin{aligned}
\mid(m+ & 1) r \frac{\partial}{\partial r} M_{m}^{(21)}(r)|+| r^{2} \frac{\partial^{2}}{\partial r^{2}} M_{m}^{(21)}(r) \mid \\
& \leqslant C\left\{\frac{m}{r^{m}} \int_{1}^{r}\left|F_{m}^{11}(\tau)\right| \tau^{m-1} \mathrm{~d} \tau+(m+1) r^{m} \int_{r}^{\infty} \frac{\left|F_{m}^{11}(\tau)\right|}{\tau^{m+1}} \mathrm{~d} \tau+\left|F_{m}^{11}(r)\right|\right\} \\
& \equiv C\left(\mathcal{Q}_{1 m}+\mathcal{Q}_{2 m}+\left|F_{m}^{11}(r)\right|\right) .
\end{aligned}
$$

It remains to estimate $L^{2}$ norms of $\mathcal{Q}_{1 m}$ and $\mathcal{Q}_{2 m}$. Substituting $\tau=r \xi$ into the integrand of $\mathcal{Q}_{2 m}$, we get

$$
\begin{aligned}
& {\left[\int_{1}^{\infty}\left|\mathcal{Q}_{2 m}(r)\right|^{2} r \mathrm{~d} r\right]^{1 / 2}} \\
& =(m+1)\left[\int_{1}^{\infty} r^{2 m}\left(\int_{1}^{\infty} \frac{F_{m}^{11}(r \xi)}{r^{m+1} \xi^{m+1}} r \mathrm{~d} \xi\right)^{2} r \mathrm{~d} r\right]^{1 / 2} \\
& =(m+1)\left[\int_{1}^{\infty}\left(\int_{1}^{\infty} \frac{F_{m}^{11}(r \xi)}{\xi^{m+1}} \mathrm{~d} \xi\right)^{2} r \mathrm{~d} r\right]^{1 / 2} \\
& \leqslant(m+1) \int_{1}^{\infty}\left(\int_{1}^{\infty}\left|F_{m}^{11}(r \xi)\right|^{2} r \mathrm{~d} r\right)^{1 / 2} \frac{\mathrm{d} \xi}{\xi^{m+1}} \quad \text { (by Minkowski's inequality) } \\
& =(m+1) \int_{1}^{\infty}\left(\int_{\xi}^{\infty}\left|F_{m}^{11}(\tau)\right|^{2} \tau \mathrm{d} \tau\right)^{1 / 2} \frac{\mathrm{d} \xi}{\xi^{m+2}} \quad(r=\tau / \xi) \\
& \leqslant\left(\int_{1}^{\infty}\left|F_{m}^{11}(\tau)\right|^{2} \tau \mathrm{d} \tau\right)^{1 / 2} \cdot
\end{aligned}
$$

Similarly, for $m \geqslant 2$ (notice that $\mathcal{Q}_{10}=0$ ),

$$
\begin{aligned}
& {\left[\int_{1}^{\infty}\left|\mathcal{Q}_{1 m}(r)\right|^{2} r \mathrm{~d} r\right]^{1 / 2}=m\left[\int_{1}^{\infty} \frac{1}{r^{2 m}}\left(\int_{1 / r}^{1} F_{m}^{11}(r \xi) r^{m-1} \xi^{m-1} r \mathrm{~d} \xi\right)^{2} r \mathrm{~d} r\right]^{1 / 2}} \\
& =m\left[\int_{1}^{\infty}\left(\int_{1 / r}^{1} F_{m}^{11}(r \xi) \xi^{m-1} \mathrm{~d} \xi\right)^{2} r \mathrm{~d} r\right]^{1 / 2} \\
& \leqslant m \int_{0}^{1}\left(\int_{1}^{\infty}\left|F_{m}^{11}(r \xi)\right|^{2} \chi_{\{r \xi>1\}} r \mathrm{~d} r\right)^{1 / 2} \xi^{m-1} \mathrm{~d} \xi \quad \text { (by Minkowski's inequality) } \\
& =m \int_{0}^{1}\left(\int_{1}^{\infty}\left|F_{m}^{11}(\tau)\right|^{2} \tau \mathrm{d} \tau\right)^{1 / 2} \xi^{m-2} \mathrm{~d} \xi \leqslant \frac{m}{m-1}\left(\int_{1}^{\infty}\left|F_{m}^{11}(\tau)\right|^{2} \tau \mathrm{d} \tau\right)^{1 / 2} .
\end{aligned}
$$

Substituting these estimates into (6.53), we obtain, for all $m \geqslant 0$,

$$
\int_{1}^{\infty}\left[(m+1) r \frac{\partial}{\partial r} M_{m}^{(21)}(r)\right]^{2} r \mathrm{~d} r+\int_{1}^{\infty}\left[r^{2} \frac{\partial^{2}}{\partial r^{2}} M_{m}^{(21)}(r)\right]^{2} r \mathrm{~d} r \leqslant C \int_{1}^{\infty}\left|F_{m}^{11}(\tau)\right|^{2} \tau \mathrm{d} \tau .
$$

This implies that

$$
\left\|r M_{r}^{(21)}\right\|_{L^{2}\left(\mathbb{R}^{2} \backslash B_{1}\right)}+\left\|r M_{r \theta}^{(21)}\right\|_{L^{2}\left(\mathbb{R}^{2} \backslash B_{1}\right)}+\left\|r^{2} M_{r r}^{(21)}\right\|_{L^{2}\left(\mathbb{R}^{2} \backslash B_{1}\right)} \leqslant C\left\|F^{11}\right\|_{L^{2}\left(\mathbb{R}^{2} \backslash B_{1}\right)} .
$$


Combining this inequality with (6.51), we get

$$
\begin{aligned}
& \left\|r M_{r}^{(2)}\right\|_{L^{2}\left(\mathbb{R}^{2} \backslash B_{1}\right)}+\left\|r M_{r \theta}^{(2)}\right\|_{L^{2}\left(\mathbb{R}^{2} \backslash B_{1}\right)}+\left\|r^{2} M_{r r}^{(2)}\right\|_{L^{2}\left(\mathbb{R}^{2} \backslash B_{1}\right)} \\
& \quad \leqslant C\left\{\left\|F^{10}\right\|_{L^{2}\left(\partial B_{1}\right)}+\left\|F^{11}\right\|_{L^{2}\left(\mathbb{R}^{2} \backslash B_{1}\right)}\right\} .
\end{aligned}
$$

Step 5. Estimating $M^{(3)}$.

Using (6.31) we see that, for $r>1$, the term with $m=0$ disappears, whereas if $m \geqslant 2$,

$$
m^{2} \int_{1}^{\infty}\left[r \frac{\partial}{\partial r} M_{m}^{(3)}(r)\right]^{2} r \mathrm{~d} r+\int_{1}^{\infty}\left[r^{2} \frac{\partial^{2}}{\partial r^{2}} M_{m}^{(3)}(r)\right]^{2} r \mathrm{~d} r \leqslant C m^{3}\left|\frac{\Lambda_{m}}{R}\right|^{2} .
$$

Therefore, by (6.45),

$$
\begin{gathered}
\left\|r M_{r}^{(3)}\right\|_{L^{2}\left(\mathbb{R}^{2} \backslash B_{1}\right)}+\left\|r M_{r \theta}^{(3)}\right\|_{L^{2}\left(\mathbb{R}^{2} \backslash B_{1}\right)}+\left\|r^{2} M_{r r}^{(3)}\right\|_{L^{2}\left(\mathbb{R}^{2} \backslash B_{1}\right)} \\
\leqslant C\left(\left\|F^{1}\right\|_{L^{2}\left(\mathbb{R}^{2}\right)}+\left\|F^{2}\right\|_{L^{2}\left(B_{1}\right)}+\left\|F^{3}\right\|_{H^{1 / 2}\left(\partial B_{1}\right)}\right) .
\end{gathered}
$$

For $r<1$,

$$
M_{m}^{(3)}(r)=\frac{\Lambda_{m}}{R} \frac{I_{m}(R r)}{I_{1}(R)} B_{m}(r) .
$$

Each $M_{m}^{(3)}$ is a power series in $r$ which does not contain a first-order term. Consequently,

$$
\left(\left|M_{m}^{(3)}\right|+\left|\frac{1}{r} \frac{\partial M_{m}^{(3)}}{\partial r}\right|+\left|\frac{\partial^{2} M_{m}^{(3)}}{\partial r^{2}}\right|\right) \leqslant C \frac{\left|\Lambda_{m}\right|}{R} \quad \text { for } \quad r<1,
$$

for each $m \geqslant 0(C$ depends on $m)$. For $m$ large, since $R s \frac{I_{m+1}(R s)}{I_{m}(R s)}=\mathrm{O}\left(\frac{1}{m}\right)$ (by (A.6)), we have

$$
\left|M_{m}^{(3)}\right| \leqslant C m \frac{\left|\Lambda_{m}\right|}{R} \int_{r}^{1}\left(\frac{r}{s}\right)^{m} s \mathrm{~d} s=\frac{C\left|\Lambda_{m}\right|}{R}\left(r^{2}-r^{m}\right) \leqslant \frac{C\left|\Lambda_{m}\right|}{R} r^{2} .
$$

Next,

$$
\begin{aligned}
\frac{\partial}{\partial r} M_{m}^{(3)} & =\frac{\Lambda_{m}}{R I_{1}(R)}\left(R I_{m}^{\prime}(R r) B_{m}(r)+I_{m}(R r) B_{m}^{\prime}(r)\right) \\
& =\frac{\Lambda_{m}}{R I_{1}(R)}\left\{R I_{m}^{\prime}(R r) B_{m}(r)+I_{1}(R r)\left(-m+R r\left[\frac{I_{0}(R r)}{I_{1}(R r)}-\frac{I_{m+1}(R r)}{I_{m}(R r)}\right]\right)\right\} .
\end{aligned}
$$

Using the relations

$$
\frac{I_{m}^{\prime}(R r)}{I_{m}(R r)}=-\frac{m}{R r}+\frac{I_{m+1}(R r)}{I_{m}(R r)}=-\frac{m}{R r}+\mathrm{O}(1), \quad B_{m}(r) I_{m}(R r)=\mathrm{O}(1),
$$

which follow by(A.6), we find that

$$
\begin{aligned}
\frac{\partial}{\partial r} M_{m}^{(3)} & =\frac{\Lambda_{m}}{R I_{1}(R)}\left(-\frac{m}{r} I_{m}(R r) B_{m}(r)-m I_{1}(R r)+\mathrm{O}(1)\right) \\
& =\frac{\Lambda_{m}}{R I_{1}(R)}\left(\frac{m^{2}}{r} \int_{r}^{1} \frac{r^{m}}{s^{m}} I_{1}(R s) \mathrm{d} s-m I_{1}(R r)+\mathrm{O}(1)\right) \quad \text { (by (6.33), }
\end{aligned}
$$


so that, by integration by parts,

$$
\frac{\partial}{\partial r} M_{m}^{(3)}=\frac{\Lambda_{m}}{R I_{1}(R)}\left(\frac{m^{2}}{m-1} \int_{r}^{1} \frac{r^{m-1}}{s^{m-1}} R I_{1}^{\prime}(R s) \mathrm{d} s-\frac{m^{2}}{m-1} I_{1}(R) r^{m-1}+\frac{m}{m-1} I_{1}(R r)+\mathrm{O}(1)\right) .
$$

Thus

$$
\left|\frac{\partial}{\partial r} M_{m}^{(3)}\right| \leqslant C \frac{\left|\Lambda_{m}\right|}{R}\left(m r^{m-1}+1\right) .
$$

Finally, by a similar (but longer) computation, we get the bound

$$
\left|\frac{\partial^{2}}{\partial r^{2}} M_{m}^{(3)}\right| \leqslant C \frac{\left|\Lambda_{m}\right|}{R}\left(m^{2} r^{m-2}+1\right) .
$$

It follows that, uniformly in $m$,

$$
\begin{aligned}
& (m+1)^{3} \int_{0}^{1}\left[M_{m}^{(3)}(r)\right]^{2} r \mathrm{~d} r+(m+1)^{2} \int_{0}^{1}\left[\frac{1}{r} \frac{\partial}{\partial r} M_{m}^{(3)}(r)\right]^{2} r \mathrm{~d} r+\int_{0}^{1}\left[\frac{\partial^{2}}{\partial r^{2}} M_{m}^{(3)}(r)\right]^{2} r \mathrm{~d} r \\
& \leqslant C(m+1)^{3}\left|\frac{\Lambda_{m}}{R}\right|^{2} .
\end{aligned}
$$

Hence

$$
\begin{aligned}
& \|\left(\left|M^{(3)}-M_{0}^{(3)}\right|+\left|\frac{1}{r} \frac{\partial\left(M^{(3)}-M_{0}^{(3)}\right)}{\partial r}\right|+\left|\frac{1}{r} \frac{\partial M^{(3)}}{\partial \theta}\right|\right. \\
& \left.+\left|\frac{\partial^{2}\left(M^{(3)}-M_{0}^{(3)}\right)}{\partial r^{2}}\right|+\left|\frac{1}{r} \frac{\partial^{2} M^{(3)}}{\partial r \partial \theta}\right|\right)\left\|_{L^{2}\left(B_{1}\right)} \leqslant C\right\| \Lambda-\Lambda_{0} \|_{H^{3 / 2}\left(\partial B_{1}\right)} .
\end{aligned}
$$

Recall that the right-hand side was already estimated in (6.46).

Step 6. Estimating $M+U$.

By (6.35),

$$
M_{m}+U_{m}=C_{3 m} r^{m}-r^{m} \mathcal{P}_{m},
$$

where $r^{m} \mathcal{P}_{m}$ can be estimated by elliptic estimates and (6.2) in the same way as $M_{m}^{(2)}$ in $r<1$. Using also the fact that $C_{2 m}=C_{3 m}$ and the bound (6.43), we conclude that

$$
\left\|M+U-C_{20}\right\|_{H^{2}\left(B_{1}\right)}
$$

is bounded by the right-hand side of (6.14).

Collecting (6.44), (6.46), (6.49), (6.50) and (6.58), the proof of (6.14) is complete, and collecting (6.47), (6.48), (6.56) and (6.57), the proof of (6.15) also follows.

Uniqueness is a by-product of the proof. Actually, for uniqueness we need only to require that the solution is even in $\theta$, that it satisfies (6.13), and that the left-hand sides of (6.14) and (6.15) are finite.

In estimating the derivatives of $\mu_{n}, u_{n}, \lambda_{n}$ inductively from the system (5.17)-(5.21), we need to have good enough estimates on the terms which appear in the $F^{j n}$ so that we can apply Lemma 6.1. But some of the terms in $F^{1 n}, F^{2 n}$ involve $\partial_{\theta}^{2} \lambda_{m}$ for $m<n$, whereas we only have $H^{3 / 2}$ estimates 
on the $\lambda_{m} \mathrm{~s}$. This means that the assumption made in Lemma 6.1 that $F^{1}, F^{2}$ belong to $L^{2}$ is too restrictive for estimating solutions corresponding to the right-hand side terms of $F^{1}, F^{2}$ which involve the $\partial_{\theta}^{2} \lambda_{m}$. Fortunately, these terms have a very special structure, to which the next lemma can be applied.

LEMma 6.2 Suppose that $F^{1}, F^{2}$ are even functions of $\theta$ such that (6.10) holds and

$$
\begin{aligned}
& F^{1}(r, \theta)=\frac{\partial}{\partial \theta} G^{1}(r, \theta) \quad \text { for } \quad r<1, \\
& F^{1}(r, \theta)=\frac{\partial}{\partial \theta} G^{1}(r, \theta)+\frac{\partial}{\partial \theta}\left(\frac{g(\theta)}{r^{2}}\right) \quad \text { for } \quad r>1, \\
& F^{2}(r, \theta)=\frac{\partial}{\partial \theta} G^{2}(r, \theta) \quad \text { for } \quad r<1, \\
& F^{3}(\theta) \equiv 0, \quad \beta=0,
\end{aligned}
$$

where $G^{1}, G^{2}, g$ are odd functions in $\theta$ which do not contain modes 1 and $l$ terms, with

$$
\begin{aligned}
J_{1}(G) \equiv & \left\|G_{1}\right\|_{L^{2}\left(B_{1}\right)}+\left\|r G_{r}^{1}\right\|_{L^{2}\left(B_{1}\right)}+\left\|r^{2} G^{1}\right\|_{L^{2}\left(\mathbb{R}^{2} \backslash B_{1}\right)}+\left\|r^{3} G_{r}^{1}\right\|_{L^{2}\left(\mathbb{R}^{2} \backslash B_{1}\right)} \\
& +\left\|G^{2}\right\|_{L^{2}\left(B_{1}\right)}+\left\|r G_{r}^{2}\right\|_{L^{2}\left(B_{1}\right)}<\infty, \\
J_{2}(G) \equiv & \left\|G_{1}(1-, \cdot)\right\|_{H^{1 / 2}\left(\partial B_{1}\right)}+\left\|G_{1}(1+, \cdot)\right\|_{H^{1 / 2}\left(\partial B_{1}\right)}+\|g\|_{H^{1 / 2}\left(\partial B_{1}\right)}<\infty .
\end{aligned}
$$

Then there exists a unique solution $(M, \Lambda, U)$ of (6.3)-(6.7) which is even in $\theta$ and satisfies (6.13) and the following inequalities:

$$
\begin{aligned}
& \|\Lambda\|_{H^{3 / 2}\left(\partial B_{1}\right)}+\|M\|_{L^{2}\left(B_{1}\right)}+\left\|\left(\left|\frac{1}{r} \frac{\partial M}{\partial r}\right|+\left|\frac{\partial^{2} M}{\partial r^{2}}\right|+\left|\frac{1}{r} \frac{\partial^{2} M}{\partial r \partial \theta}\right|\right)\right\|_{L^{2}\left(B_{1}\right)} \\
& \quad+\|M+U\|_{L^{2}\left(B_{1}\right)}+\left\|\left(\left|\frac{1}{r} \frac{\partial(M+U)}{\partial r}\right|+\left|\frac{\partial^{2}(M+U)}{\partial r^{2}}\right|+\left|\frac{1}{r} \frac{\partial^{2}(M+U)}{\partial r \partial \theta}\right|\right)\right\|_{L^{2}\left(B_{1}\right)} \\
& \leqslant C\left(J_{1}(G)+J_{2}(G)\right),
\end{aligned}
$$

$$
\begin{aligned}
& \left\|r^{2}\left(\left|\frac{1}{r} \frac{\partial M}{\partial r}\right|+\left|\frac{\partial^{2} M}{\partial r^{2}}\right|+\left|\frac{1}{r} \frac{\partial^{2} M}{\partial r \partial \theta}\right|\right)\right\|_{L^{2}\left(\mathbb{R}^{2} \backslash B_{1}\right)} \\
& \leqslant C\left(J_{1}(G)+J_{2}(G)\right) .
\end{aligned}
$$

REMARK 6.4 It would appear more consistent with the statement of Lemma 6.1 to replace $G^{1}(r, \theta)$ by $G^{1}(r, \theta) / r^{2}$ for $r>1$, and similarly change

$$
\left\|r^{2} G^{1}\right\|_{L^{2}\left(\mathbb{R}^{2} \backslash B_{1}\right)} \quad \text { to } \quad\left\|G^{1}\right\|_{L^{2}\left(\mathbb{R}^{2} \backslash B_{1}\right)} \text { etc. }
$$

However, the present notation is more convenient for the subsequent applications of the lemma.

Proof. Proceeding as in the proof of Lemma 6.1, we can solve $M^{(1)}, M^{(2)}, M^{(3)}, M+U, \Lambda$ as before. Since $F^{1}, F^{2}$ are no longer in $L^{2}$, we are forced to estimate the integrals in (6.43) in a different way. Since $F^{3} \equiv 0, \beta=0$, mode 0 terms also do not appear; by (6.10) also no modes 1 
and $l$ appear in the solution, and thus we need to consider only modes $m \geqslant 2, m \neq l$. In particular, $M_{0}(r) \equiv 0, M_{0}^{(3)}(r) \equiv 0$ and therefore $C_{20}=0$.

Step 1. Estimate for $M^{(1)}$.

For $r>1$,

$$
\begin{aligned}
F_{m}^{1}(r) & =\frac{1}{\pi} \int_{0}^{2 \pi} F^{1}(r, \theta) \cos m \theta \\
& =\frac{-m}{\pi} \int_{0}^{2 \pi}\left(G^{1}(r, \theta)+\frac{g(\theta)}{r^{2}}\right) \sin m \theta \mathrm{d} \theta \equiv-m\left[G_{m}^{1}(r)+\frac{1}{r^{2}} g_{m}\right],
\end{aligned}
$$

so that, if $m \geqslant 3$,

$$
\begin{aligned}
\mid \int_{1}^{\infty} & \frac{F_{m}^{1}(r)}{r^{m-1}} \mathrm{~d} r \mid \\
\quad & =\left|\frac{1}{m-2} F_{m}^{1}(1+)+\frac{1}{m-2} \int_{1}^{\infty} r^{2-m} \frac{\partial}{\partial r} F_{m}^{1}(r) \mathrm{d} r\right| \\
& =\left|\frac{-m}{m-2}\left[G_{m}^{1}(1+)+g_{m}\right]-\frac{m}{m-2} \int_{1}^{\infty} r^{2-m}\left(\frac{\partial}{\partial r} G_{m}(r)-\frac{2}{r^{3}} g_{m}\right) \mathrm{d} r\right| \\
& \leqslant 3\left|G_{m}^{1}(1+)+g_{m}\right|+3\left[\left(\int_{1}^{\infty}\left[r \frac{\partial}{\partial r} G_{m}(r)\right]^{2} r \mathrm{~d} r\right)^{1 / 2}+2\left|g_{m}\right|\right]\left(\int_{1}^{\infty} r^{-2 m} r \mathrm{~d} r\right)^{1 / 2} \\
& \leqslant 3\left|G_{m}^{1}(1+)\right|+9\left|g_{m}\right|+\frac{6}{m^{1 / 2}}\left\|r \frac{\partial}{\partial r} G_{m}(r)\right\|_{L^{2}\left(\mathbb{R}^{2} \backslash B_{1}\right)}
\end{aligned}
$$

whereas if $m=2$,

$$
\left|\int_{1}^{\infty} \frac{F_{m}^{1}(r)}{r^{m-1}} \mathrm{~d} r\right| \leqslant\left\|F_{2}^{1}\right\|_{L^{2}\left(\mathbb{R}^{2} \backslash B_{1}\right)} \leqslant 2\left(\left\|G_{2}^{1}(r)\right\|_{L^{2}\left(\mathbb{R}^{2} \backslash B_{1}\right)}+\left|g_{2}\right|\right) .
$$

Similarly, for all $r<1, m \geqslant 2$,

$$
\begin{aligned}
\left|\int_{0}^{1} \frac{I_{m}(R \tau)}{I_{m}(R)} F_{m}^{1}(\tau) \tau \mathrm{d} \tau\right| & =\left|F_{m}^{1}(1-) \int_{0}^{1} \frac{I_{m}(R \tau)}{I_{m}(R)} \tau \mathrm{d} \tau-\int_{0}^{1}\left(\int_{0}^{r} \frac{I_{m}(R \tau)}{I_{m}(R)} \tau \mathrm{d} \tau\right) \frac{\partial}{\partial r} F_{m}^{1}(r) \mathrm{d} r\right| \\
& \leqslant \frac{C}{m}\left|F_{m}^{1}(1-)\right|+\frac{C}{m} \int_{0}^{1} r^{m+2}\left|\frac{\partial}{\partial r} F_{m}^{1}(r)\right| \mathrm{d} r \\
& \leqslant C\left|G_{m}^{1}(1-)\right|+\frac{C}{m^{1 / 2}} \mid r \frac{\partial}{\partial r} G_{m}^{1}(r) \|_{L^{2}\left(B_{1}\right)}
\end{aligned}
$$

We can estimate $\int_{0}^{1} \tau^{m}\left(F_{m}^{1}(\tau)+F_{m}^{2}(\tau)\right) \tau \mathrm{d} \tau$ in the same manner. Substituting these estimates into the first inequality in (6.43) and recalling that $F_{3}=0$, we get

$$
\begin{aligned}
m\left(\left|\frac{\Lambda_{m}}{R}\right|+\left|C_{2 m}\right|\right) \leqslant & C\left(\left|G_{m}^{1}(1 \pm)\right|+\left|g_{m}\right|\right) \\
& +\frac{C}{m^{1 / 2}}\left\{\left\|r \frac{\partial}{\partial r} G_{m}(r)\right\|_{L^{2}\left(\mathbb{R}^{2} \backslash B_{1}\right)}+\left\|r \frac{\partial}{\partial r} G_{m}(r)\right\|_{L^{2}\left(B_{1}\right)}\right\} .
\end{aligned}
$$


Using this estimate (instead of (6.43)), we can then follow the proof of (6.45)-(6.49) and derive the bounds

$$
\begin{gathered}
\|\Lambda\|_{H^{3 / 2}\left(\partial B_{1}\right)} \leqslant C\left(\left\|r G_{r}^{1}\right\|_{L^{2}\left(B_{1}\right)}+\left\|r G_{r}^{1}\right\|_{L^{2}\left(\mathbb{R}^{2} \backslash B_{1}\right)}+\left\|G^{1}(1+, \cdot)\right\|_{H^{1 / 2}\left(\partial B_{1}\right)}\right. \\
\left.+\left\|G^{1}(1-, \cdot)\right\|_{H^{1 / 2}\left(\partial B_{1}\right)}+\|g\|_{H^{1 / 2}\left(\partial B_{1}\right)}\right) \\
\left\|r M_{r}^{(1)}\right\|_{L^{2}\left(\mathbb{R}^{2} \backslash B_{1}\right)}+\left\|r M_{r \theta}^{(1)}\right\|_{L^{2}\left(\mathbb{R}^{2} \backslash B_{1}\right)}+\left\|r^{2} M_{r r}^{(1)}\right\|_{L^{2}\left(\mathbb{R}^{2} \backslash B_{1}\right)} \\
\leqslant C\left(\left\|r G_{r}^{1}\right\|_{L^{2}\left(B_{1}\right)}+\left\|r G_{r}^{1}\right\|_{L^{2}\left(\mathbb{R}^{2} \backslash B_{1}\right)}+\left\|G^{1}(1+, \cdot)\right\|_{H^{1 / 2}\left(\partial B_{1}\right)}\right. \\
\left.+\left\|G^{1}(1-, \cdot)\right\|_{H^{1 / 2}\left(\partial B_{1}\right)}+\|g\|_{H^{1 / 2}\left(\partial B_{1}\right)}\right)
\end{gathered}
$$

and

$$
\begin{aligned}
\left\|M^{(1)}\right\|_{H^{2}\left(B_{1}\right)} \leqslant & C\left(\left\|r G_{r}^{1}\right\|_{L^{2}\left(B_{1}\right)}+\left\|r G_{r}^{1}\right\|_{L^{2}\left(\mathbb{R}^{2} \backslash B_{1}\right)}+\left\|G^{1}(1, \cdot)\right\|_{H^{1 / 2}\left(\partial B_{1}\right)}\right. \\
& \left.+\|g\|_{H^{1 / 2}\left(\partial B_{1}\right)}\right) .
\end{aligned}
$$

Step 2. Estimate for $M^{(2)}$.

Let $W$ be the solution of

$$
\Delta W-R^{2} W=G^{1}(r, \theta) \quad \text { in } \quad B_{1}, \quad W=0 \quad \text { on } \partial B_{1} .
$$

Then

$$
M^{(2)}=\frac{\partial}{\partial \theta} W \quad \text { in } \quad B_{1} .
$$

By elliptic estimates and (6.2),

$$
\|W\|_{H_{r}^{2}\left(B_{1}\right)} \leqslant C\|W\|_{H^{2}\left(B_{1}\right)} \leqslant C\left\|G^{1}\right\|_{L^{2}\left(B_{1}\right)} .
$$

A direct computation shows that the function $\varphi(r, \theta)=r W_{r}$ satisfies

$$
\begin{aligned}
& \Delta \varphi-R^{2} \varphi=r G_{r}^{1}+2 W_{r r}+\frac{2}{r} W_{r}+\frac{2}{r^{2}} W_{\theta \theta} \equiv \mathcal{G} \quad \text { in } \quad B_{1}, \\
& \varphi_{r}=G^{1}(1, \theta) \quad \text { on } \quad \partial B_{1},
\end{aligned}
$$

and, by (6.2) and (6.72),

$$
\|\mathcal{G}\|_{L^{2}\left(B_{1}\right)} \leqslant C\left(\left\|G^{1}\right\|_{L^{2}\left(B_{1}\right)}+\left\|r G_{r}^{1}\right\|_{L^{2}\left(B_{1}\right)}\right) .
$$

Therefore, by $L^{2}$ elliptic estimates for the Neumann boundary value problem and by (6.2),

$$
\begin{aligned}
\left\|r W_{r}\right\|_{H_{r}^{2}\left(B_{1}\right)} & \leqslant C\left\|r W_{r}\right\|_{H^{2}\left(B_{1}\right)}=C\|\varphi\|_{H^{2}\left(B_{1}\right)} \\
& \leqslant C\left(\left\|G^{1}\right\|_{L^{2}\left(B_{1}\right)}+\left\|r G_{r}^{1}\right\|_{L^{2}\left(B_{1}\right)}+\left\|G^{1}(1-, \cdot)\right\|_{H^{1 / 2}\left(\partial B_{1}\right)}\right) .
\end{aligned}
$$


One can readily check that

$$
\begin{aligned}
& \left\|M^{(2)}\right\|_{L^{2}\left(B_{1}\right)}+\left\|M_{r r}^{(2)}\right\|_{L^{2}\left(B_{1}\right)}+\left\|\frac{1}{r} M_{r}^{(2)}\right\|_{L^{2}\left(B_{1}\right)}+\left\|\frac{1}{r} M_{r \theta}^{(2)}\right\|_{L^{2}\left(B_{1}\right)} \\
& \leqslant C\left(\left\|r\left(\frac{1}{r} W_{\theta}\right)\right\|_{L^{2}\left(B_{1}\right)}+\left\|\frac{1}{r}\left(r W_{r}\right)_{r \theta}\right\|_{L^{2}\left(B_{1}\right)}+\left\|\frac{1}{r} W_{r \theta}\right\|_{L^{2}\left(B_{1}\right)}+\left\|\frac{1}{r^{2}}\left(r W_{r}\right)_{\theta \theta}\right\|_{L^{2}\left(B_{1}\right)}\right) \\
& \leqslant C\left(\left\|G^{1}\right\|_{L^{2}\left(B_{1}\right)}+\left\|r G_{r}^{1}\right\|_{L^{2}\left(B_{1}\right)}+\left\|G^{1}(1-, \cdot)\right\|_{H^{1 / 2}\left(\partial B_{1}\right)}\right),
\end{aligned}
$$

where the second inequality is a consequence of (6.72) and (6.75).

Next we estimate $M^{(2)}$ for $r>1$. We use the formula (6.30), as before. Let

$$
\begin{aligned}
& F_{m}^{1}(r)=\frac{1}{r^{2}} F_{m}^{10}+F_{m}^{11}(r), \\
& F_{m}^{10}=\frac{1}{\pi} \int_{0}^{2 \pi} g^{\prime}(\theta) \cos m \theta \mathrm{d} \theta=\frac{-m}{\pi} \int_{0}^{2 \pi} g(\theta) \sin m \theta \mathrm{d} \theta=-m g_{m}, \\
& F_{m}^{11}(r)=\frac{1}{\pi} \int_{0}^{2 \pi} G_{\theta}^{1}(r, \theta) \cos m \theta \mathrm{d} \theta=\frac{-m}{\pi} \int_{0}^{2 \pi} G^{1}(r, \theta) \sin m \theta \mathrm{d} \theta=-m G_{m}^{1}(r) .
\end{aligned}
$$

We can then write $M^{(2)}=M^{(20)}+M^{(21)}$, where

$$
M^{(20)}(r, \theta)=\sum_{m \geqslant 2} M_{m}^{(20)}(r) \cos m \theta, \quad M^{(21)}(r, \theta)=\sum_{m \geqslant 2} M_{m}^{(21)}(r) \cos m \theta,
$$

and

$$
\begin{aligned}
& M_{m}^{(20)}(r)=-\frac{1}{r^{m}} \int_{1}^{r} s^{2 m-1} \int_{s}^{\infty} \frac{F^{10}}{\tau^{m+1}} \mathrm{~d} \tau=-\frac{F^{10}}{m^{2}}\left(1-\frac{1}{r^{m}}\right), \\
& M_{m}^{(21)}(r)=-\frac{1}{r^{m}} \int_{1}^{r} s^{2 m-1} \int_{s}^{\infty} \frac{F_{m}^{11}(\tau)}{\tau^{m-1}} \mathrm{~d} \tau .
\end{aligned}
$$

As before, we have

$$
m^{2} \int_{1}^{\infty}\left(r \frac{\partial}{\partial r} M_{m}^{(20)}(r)\right)^{2} r \mathrm{~d} r+\int_{1}^{\infty}\left(r^{2} \frac{\partial^{2}}{\partial r^{2}} M_{m}^{(20)}(r)\right)^{2} r \mathrm{~d} r \leqslant \frac{C}{m}\left|F_{m}^{10}\right|^{2} \leqslant C m\left|g_{m}\right|^{2},
$$

so that

$$
\left\|r M_{r}^{(20)}\right\|_{L^{2}\left(\mathbb{R}^{2} \backslash B_{1}\right)}+\left\|r M_{r \theta}^{(20)}\right\|_{L^{2}\left(\mathbb{R}^{2} \backslash B_{1}\right)}+\left\|r^{2} M_{r r}^{(20)}\right\|_{L^{2}\left(\mathbb{R}^{2} \backslash B_{1}\right)} \leqslant C\|g\|_{H^{1 / 2}\left(\partial B_{1}\right)} .
$$

Next, by direct computation as in the previous lemma,

$$
\begin{aligned}
\frac{\partial}{\partial r} M_{m}^{(21)}(r)= & \frac{m}{r^{m+1}} \int_{1}^{r} s^{2 m-1} \int_{s}^{\infty} \frac{F_{m}^{11}(\tau)}{\tau^{m-1}} \mathrm{~d} \tau-r^{m-1} \int_{r}^{\infty} \frac{F_{m}^{11}(\tau)}{\tau^{m-1}} \mathrm{~d} \tau \\
\frac{\partial^{2}}{\partial r^{2}} M_{m}^{(21)}(r)= & -\frac{m(m+1)}{r^{m+2}} \int_{1}^{r} s^{2 m-1} \int_{s}^{\infty} \frac{F_{m}^{11}(\tau)}{\tau^{m-1}} \mathrm{~d} \tau \\
& +r^{m-2} \int_{r}^{\infty} \frac{F_{m}^{11}(\tau)}{\tau^{m-1}} \mathrm{~d} \tau+F_{m}^{11}(r) .
\end{aligned}
$$


By integration by parts, we obtain, for $m \geqslant 3$,

$$
\begin{gathered}
\int_{r}^{\infty} \frac{F_{m}^{11}(\tau)}{\tau^{m-1}} \mathrm{~d} \tau \\
=\frac{r^{2-m}}{m-2} F_{m}^{11}(r)+\frac{1}{m-2} \int_{r}^{\infty} \tau^{2-m} \frac{\partial}{\partial \tau} F_{m}^{11}(\tau) \mathrm{d} \tau \\
=-\frac{m}{m-2} r^{2-m} G_{m}^{1}(r)-\frac{m}{m-2} \int_{r}^{\infty} \tau^{2-m} \frac{\partial}{\partial \tau} G_{m}^{1}(\tau) \mathrm{d} \tau, \\
\int_{1}^{r} s^{2 m-1} \int_{s}^{\infty} \frac{F_{m}^{11}(\tau)}{\tau^{m-1}} \mathrm{~d} \tau \\
=-\frac{m}{m-2} \int_{1}^{r} s^{m+1} G_{m}^{1}(s) \mathrm{d} s-\frac{m}{m-2} \int_{1}^{r} s^{2 m-1} \int_{s}^{\infty} \tau^{2-m} \frac{\partial}{\partial \tau} G_{m}^{1}(\tau) \mathrm{d} \tau \\
=\frac{m}{m^{2}-4}\left[G_{m}^{1}(1)-r^{m+2} G_{m}^{1}(r)\right]+\frac{m}{m^{2}-4} \int_{1}^{r} \tau^{m+2} \frac{\partial}{\partial \tau} G_{m}^{1}(\tau) \mathrm{d} \tau \\
-\frac{m}{m-2} \int_{1}^{r} s^{2 m-1} \int_{s}^{\infty} \tau^{2-m} \frac{\partial}{\partial \tau} G_{m}^{1}(\tau) \mathrm{d} \tau .
\end{gathered}
$$

Substituting these equalities into (6.79), (6.80) we find that the $\mathrm{O}\left(m^{0}\right)$ order terms of $r G_{m}^{1}(r)$ in $\partial M_{m}^{(21)}(r) / \partial r$ and $\mathrm{O}\left(m^{1}\right)$ order terms of $G_{m}^{1}(r)$ in $\partial^{2} M_{m}^{(21)}(r) / \partial r^{2}$ cancel out, and we obtain

$$
\begin{aligned}
\left|m r \frac{\partial}{\partial r} M_{m}^{(21)}(r)\right|+\left|r^{2} \frac{\partial^{2}}{\partial r^{2}} M_{m}^{(21)}(r)\right| & \\
\leqslant & C\left\{\frac{m^{2}}{r^{m}} \int_{1}^{r} s^{2 m-1} \int_{s}^{\infty} \tau^{2-m}\left|\frac{\partial}{\partial \tau} G_{m}^{1}(\tau)\right| \mathrm{d} \tau+m r^{m} \int_{r}^{\infty} \tau^{2-m}\left|\frac{\partial}{\partial \tau} G_{m}^{1}(\tau)\right| \mathrm{d} \tau\right. \\
& \left.+\frac{m}{r^{m}} \int_{1}^{r} \tau^{m+2}\left|\frac{\partial}{\partial \tau} G_{m}^{1}(\tau)\right| \mathrm{d} \tau+\frac{m}{r^{m}} G_{m}^{1}(1)+\left|r^{2} G_{m}^{1}(r)\right|\right\} \\
\equiv & C\left(\mathcal{Q}_{1 m}+\mathcal{Q}_{2 m}+\mathcal{Q}_{3 m}+\frac{m}{r^{m}} G_{m}^{1}(1)+\left|r^{2} G_{m}^{1}(r)\right|\right) .
\end{aligned}
$$

The same computation as in (6.52) yields the estimate $\mathcal{Q}_{1 m} \leqslant \mathcal{Q}_{2 m}+\mathcal{Q}_{3 m}$. Following the same procedure as in Lemma 6.1, we obtain

$$
\int_{1}^{\infty}\left[\mathcal{Q}_{2 m}(r)\right]^{2} r \mathrm{~d} r+\int_{1}^{\infty}\left[\mathcal{Q}_{3 m}(r)\right]^{2} r \mathrm{~d} r \leqslant C \int_{1}^{\infty}\left|\tau^{3} \frac{\partial}{\partial \tau} G_{m}^{1}(\tau)\right|^{2} \tau \mathrm{d} \tau .
$$

Thus, for $m \geqslant 3$,

$$
\begin{array}{r}
m^{2} \int_{1}^{\infty}\left[r \frac{\partial}{\partial r} M_{m}^{(21)}(r)\right]^{2} r \mathrm{~d} r+\int_{1}^{\infty}\left[r^{2} \frac{\partial^{2}}{\partial r^{2}} M_{m}^{(21)}(r)\right]^{2} r \mathrm{~d} r \\
\leqslant C\left\{m\left[G_{m}^{1}(1)\right]^{2}+\int_{1}^{\infty}\left|\tau^{2} G_{m}^{1}(\tau)\right|^{2} \tau \mathrm{d} \tau+\int_{1}^{\infty}\left|\tau^{3} \frac{\partial}{\partial \tau} G_{m}^{1}(\tau)\right|^{2} \tau \mathrm{d} \tau\right\} .
\end{array}
$$

For $m=2,\left|F_{2}^{11}(r)\right|=2\left|G_{2}^{1}(r)\right|$, and we just have to slightly modify the above proof in order to derive the same inequality. Summing over $m \geqslant 2$, we deduce that

$$
\begin{aligned}
& \left\|r M_{r}^{(21)}\right\|_{L^{2}\left(\mathbb{R}^{2} \backslash B_{1}\right)}+\left\|r M_{r \theta}^{(21)}\right\|_{L^{2}\left(\mathbb{R}^{2} \backslash B_{1}\right)}+\left\|r^{2} M_{r r}^{(21)}\right\|_{L^{2}\left(\mathbb{R}^{2} \backslash B_{1}\right)} \\
\leqslant & C\left[\left\|G^{1}(1+, \cdot)\right\|_{H^{1 / 2}\left(\partial B_{1}\right)}+\left\|r^{2} G^{1}\right\|_{L^{2}\left(\mathbb{R}^{2} \backslash B_{1}\right)}+\left\|r^{3} G_{r}^{1}\right\|_{L^{2}\left(\mathbb{R}^{2} \backslash B_{1}\right)}\right] .
\end{aligned}
$$


Step 3. Estimate for $M^{(3)}$.

The estimate for $M^{(3)}$ proceeds as in (6.58) with $\left\|\Lambda-\Lambda_{0}\right\|_{H^{3 / 2}}$ replaced by $\|\Lambda\|_{H^{3 / 2}}$.

Step 4. Estimate for $M+U$.

This is similar to the estimate in Lemma 6.1, but using the technique in Step 2 of this lemma for estimating $M^{(2)}$ in $r<1$.

Combining the above estimates, the proof of the lemma is complete.

We next consider the case of mode $l$ only, namely

$$
\begin{aligned}
& F^{1}(r, \theta)=F_{l}^{1}(r) \cos l \theta \quad \text { for } \quad r<1, \\
& F^{1}(r, \theta)=\frac{1}{r^{2}} F_{l}^{10} \cos l \theta+\frac{1}{r^{2}} F_{l}^{11}(r) \cos l \theta, \quad \text { for } \quad r>1, \\
& F^{2}(r, \theta)=F_{l}^{2}(r) \cos l \theta \quad \text { for } \quad r<1, \quad F^{3}(\theta)=F_{l}^{3} \cos l \theta .
\end{aligned}
$$

As before, we obtain a linear system of equations for $\left(C_{1 l}, C_{2 l}, C_{3 l}, \Lambda_{l} / R\right)$. Since in this case the determinant of the coefficients matrix of (6.40), (6.41) (with $m=l$ ) is zero, this system can be solved if and only if the following compatibility condition is satisfied:

$$
\operatorname{det}\left(\begin{array}{cc}
\frac{R I_{l-1}(R)}{I_{l}(R)}, & -\int_{1}^{\infty} \frac{F_{l}^{1}(\tau)}{\tau^{l-1}} \mathrm{~d} \tau-\frac{1}{I_{l}(R)} \int_{0}^{1} \tau I_{l}(R \tau) F_{l}^{1}(\tau) \mathrm{d} \tau \\
2 l, & F_{l}^{3}-\int_{0}^{1} \tau^{l+1}\left\{F_{l}^{1}(\tau)+F_{l}^{2}(\tau)\right\} \mathrm{d} \tau-\int_{1}^{\infty} \frac{F_{l}^{1}(\tau)}{\tau^{l-1}} \mathrm{~d} \tau
\end{array}\right)=0 .
$$

The solution is unique up to a multiple by a homogeneous solution. Hence, if we impose the condition $\Lambda_{l}=0$ then the solution of (6.40), (6.41) is unique. For later references we state the following lemma.

Lemma 6.3 For the data (6.84) (mode $l$ ) a solution which is even in $\theta$ exists if and only if

$$
\begin{aligned}
(2 l- & \left.R \frac{I_{l-1}(R)}{I_{l}(R)}\right) \int_{1}^{\infty} \frac{F_{l}^{1}(\tau)}{\tau^{l-1}} \mathrm{~d} \tau+\frac{2 l}{I_{l}(R)} \int_{0}^{1} \tau I_{l}(R \tau) F_{l}^{1}(\tau) \mathrm{d} \tau \\
& +R \frac{I_{l-1}(R)}{I_{l}(R)}\left[F_{l}^{3}-\int_{0}^{1} \tau^{l+1}\left\{F_{l}^{1}(\tau)+F_{l}^{2}(\tau)\right\} \mathrm{d} \tau\right]=0,
\end{aligned}
$$

and the solution is uniquely determined by the condition $\Lambda_{l}=0$.

The next lemma summarizes Lemmas 6.1-6.3.

Lemma 6.4 Assume that in (6.3),(6.4), $F^{j}=\widehat{F}^{j}+\widetilde{F}^{j}+\bar{F}^{j}$, where $\widehat{F}^{j}, \widetilde{F}^{j}$ and $\bar{F}^{j}$ satisfy the assumptions of Lemmas 6.1-6.3, respectively, i.e.

(a) $\widehat{F}^{j}$ and $\widetilde{F}^{j}$ do not contain modes 1 and $l ; \bar{F}^{j}$ contains mode $l$ only.

(b) $\widehat{F}^{j}$ satisfies the conditions of Lemma 6.1 and $\widetilde{F}^{j}$ satisfies the conditions of Lemma 6.2.

If the compatibility condition (6.85) is satisfied for the $\bar{F}^{j}$, then there exists a unique solution $(M, U, \Lambda)$ which is even in $\theta$, satisfies the condition $\Lambda_{l}=0$ and can be decomposed into a sum of three parts, satisfying, respectively, the properties asserted in Lemmas 6.1-6.3.

REMARK 6.5 If we differentiate the equations (6.3)-(6.7) in $\theta$, we get an equation which is exactly the same except that the solutions will be odd in $\theta$ and we need to work with Fourier sine series. The conclusion in Lemmas 6.1-6.4 are still valid (with the estimates and the right-hand sides replaced by their $\theta$-derivative). If we differentiate in $\theta$ a second time, the solutions will become even in $\theta$ and Lemmas 6.1-6.4 can again be directly applied. 


\section{Convergence}

In this section we estimate inductively the $\left(\mu_{n}, u_{n}, \lambda_{n}, \beta_{n-1}\right)$. We denote the mode $k$ of $F^{j n}$ in (5.17), (5.18), (5.21) by $F_{k}^{j n}$. By Lemma 6.4 the system (5.17)-(5.21) can be uniquely solved for any mode $k, k \neq l$. For mode $l$ the system can be solved if and only if the compatibility condition (6.85) is satisfied. This condition will take the form $\widetilde{\Lambda} \beta_{n-1}=\widetilde{F}_{n}$ where $\widetilde{F}_{n}$ is determined by the $\mu_{s}, u_{s}, \lambda_{s}, \beta_{s-1}$ for $s<n$ and, as the next lemma shows, $\widetilde{\Lambda} \neq 0$ and $\widetilde{\Lambda}$ is independent of $n$.

LEMma 7.1 The expression

$$
\begin{aligned}
\widetilde{\Lambda} \equiv & \frac{\partial}{\partial \beta_{n-1}}\left\{\left(2 l-R \frac{I_{l-1}(R)}{I_{l}(R)}\right) \int_{1}^{\infty} \frac{F_{l}^{1 n}(\tau)}{\tau^{l-1}} \mathrm{~d} \tau+\frac{2 l}{I_{l}(R)} \int_{0}^{1} \tau I_{l}(R \tau) F_{l}^{1 n}(\tau) \mathrm{d} \tau\right. \\
& \left.+R \frac{I_{l-1}(R)}{I_{l}(R)}\left[F_{l}^{3 n}-\int_{0}^{1} \tau^{l+1}\left\{F_{l}^{1 n}(\tau)+F_{l}^{2 n}(\tau)\right\} \mathrm{d} \tau\right]\right\}
\end{aligned}
$$

is independent of $n$ and is nonzero.

Proof. The compatibility condition is invariant under the change of variable (5.1). Therefore the assertion that $\widetilde{\Lambda} \neq 0$ (which is equivalent to the statement that the compatibility condition (6.85) holds for some choice of $\beta_{n-1}$ ) follows from Theorem 4.3. We next proceed to derive an expression for $\widetilde{\Lambda}$ which, although very complicated, shows that it is independent of $n$ (this fact does not follow from Theorem 4.3). We begin by writing explicit formulae for $F^{j n}$. Substituting (5.2), (5.9)-(5.12) into (5.4), (5.5), (5.8), we find that $\left(\mu_{n}, u_{n}, \lambda_{n}, \beta_{n}\right)=(M, U, \Lambda, \beta)$ satisfy (6.3)-(6.7) with $F^{j}=$ $F^{j n}$ defined as the $\varepsilon^{n}$ order terms in the following expansion:

$$
\begin{aligned}
\sum_{n \geqslant 1} \varepsilon^{n} F^{1 n}= & \left(-\frac{f_{\theta \theta}}{R+f}+\frac{f_{\theta \theta}}{R}\right) \frac{1}{r} \frac{\partial \mu_{0}}{\partial r}+\frac{f_{\theta}^{2}}{(R+f)^{2}}\left(\frac{\partial^{2} \mu_{0}}{\partial r^{2}}+\frac{2}{r} \frac{\partial \mu_{0}}{\partial r}\right) \\
& -\frac{f_{\theta \theta}}{R+f} \sum_{n \geqslant 1} \varepsilon^{n} \frac{1}{r} \frac{\partial \mu_{n}}{\partial r}-\frac{2 f_{\theta}}{R+f} \sum_{n \geqslant 1} \varepsilon^{n} \frac{1}{r} \frac{\partial^{2} \mu_{n}}{\partial \theta \partial r} \\
& +\frac{f_{\theta}^{2}}{(R+f)^{2}} \sum_{n \geqslant 1} \varepsilon^{n}\left(\frac{\partial^{2} \mu_{n}}{\partial r^{2}}+\frac{2}{r} \frac{\partial \mu_{n}}{\partial r}\right) \\
& -\chi_{\{r<1\}}\left(\left(2 R f+f^{2}\right) \sum_{n \geqslant 1} \varepsilon^{n} \mu_{n}+f^{2} \mu_{0}\right), \\
\sum_{n \geqslant 1} \varepsilon^{n}\left(F^{1 n}+F^{2 n}\right)= & -\frac{f_{\theta \theta}}{R+f} \sum_{n \geqslant 1} \varepsilon^{n} \frac{1}{r} \frac{\partial\left(\mu_{n}+u_{n}\right)}{\partial r}-\frac{2 f_{\theta}}{R+f} \sum_{n \geqslant 1} \varepsilon^{n} \frac{1}{r} \frac{\partial^{2}\left(\mu_{n}+u_{n}\right)}{\partial \theta \partial r} \\
& +\frac{f_{\theta}^{2}}{(R+f)^{2}} \sum_{n \geqslant 1} \varepsilon^{n}\left(\frac{\partial^{2}\left(\mu_{n}+u_{n}\right)}{\partial r^{2}}+\frac{2}{r} \frac{\partial\left(\mu_{n}+u_{n}\right)}{\partial r}\right), \\
-\sum_{n \geqslant 1} \varepsilon^{n} F^{3 n}= & \left(\sum_{n \geqslant 0} \varepsilon^{n} \beta_{n}\right)\left[\frac{(R+f)^{2}}{\left[(R+f)^{2}+f_{\theta}^{2}\right]^{1 / 2}}-R\right]-\frac{1}{R} f .
\end{aligned}
$$

Consider the system for $\left(\mu_{n-1}, u_{n-1}, \lambda_{n-1}\right)$, whose solution is given in Section 6 (Lemma 6.4) with $F^{j}=F^{j, n-1}$. The solution depends on $\beta_{n-1}$ only through its zero mode (see (6.36)-(6.39) 
with $\beta=\beta_{n-1}$ and note that $\delta_{m 0} \beta=0$ if $m>0$ ). We need to find the explicit dependence of the $F_{m}^{j n}$ on $\beta_{n-1}$. We therefore first solve the system (6.36)-(6.39) with $F_{m}^{j}=F_{m}^{j, n-1}, m=0$ and $\beta=\beta_{n-1}$. By Remark 6.2, if $m=0$ then $I_{m-1}$ in (6.37) is replaced by $I_{1}$. Solving (6.40), (6.41) and (6.36), (6.38), we find

$$
\begin{aligned}
& \frac{1}{R} \Lambda_{0}^{n-1}=-\beta_{n-1} R+\cdots, \\
& C_{20}^{n-1}=C_{30}^{n-1}=\beta_{n-1} R\left(\frac{I_{0}^{2}(R)}{I_{1}^{2}(R)}-1\right)+\cdots, \\
& C_{10}^{n-1}=\beta_{n-1} \frac{R}{I_{0}(R)}\left(\frac{I_{0}^{2}(R)}{I_{1}^{2}(R)}-1\right)+\cdots,
\end{aligned}
$$

where '.. ' refers to terms independent of $\beta_{n-1}$. Then, from the formula for $M=\mu_{n-1}$ in Lemma 6.4 (or actually the special case stated as Lemma 6.1),

$$
\begin{aligned}
& \frac{\partial}{\partial \beta_{n-1}} \mu_{n-1}=I_{0}(R r) \frac{\partial C_{10}^{n-1}}{\partial \beta_{n-1}}+\frac{I_{0}(R r)}{I_{1}(R)} B_{0}(r) \frac{\partial}{\partial \beta_{n-1}} \frac{\Lambda_{0}^{n-1}}{R} \\
& \quad=\frac{R I_{0}(R r)}{I_{0}(R)}\left\{\frac{I_{0}^{2}(R)}{I_{1}^{2}(R)}-1-\frac{I_{0}(R)}{I_{1}(R)} B_{0}(r)\right\} \\
& \quad=\frac{R I_{0}(R) I_{0}(R r)}{I_{1}^{2}(R)}-\frac{R r}{I_{1}(R)} I_{1}(R r) \quad \text { for } r<1, \\
& \left.\frac{\partial}{\partial \beta_{n-1}} \mu_{n-1}=\frac{\partial}{\partial \beta_{n-1}} C_{20}^{n-1}=R\left(\frac{I_{0}^{2}(R)}{I_{1}^{2}(R)}-1\right) \quad \text { for } r>1 \quad \text { (since } M_{0}^{(3)} \equiv 0\right), \\
& \frac{\partial}{\partial \beta_{n-1}}\left(\mu_{n-1}+u_{n-1}\right)=\frac{\partial}{\partial \beta_{n-1}} C_{30}^{n-1}=R\left(\frac{I_{0}^{2}(R)}{I_{1}^{2}(R)}-1\right) \quad \text { for } r<1, \\
& \left.\frac{1}{(n-1) !} \frac{\partial}{\partial \beta_{n-1}} \frac{\partial^{n-1}}{\partial \varepsilon^{n-1}}\right|_{\varepsilon=0} f=\frac{\partial}{\partial \beta_{n-1}} \lambda_{n-1}=\frac{\partial}{\partial \beta_{n-1}} \Lambda_{0}^{n-1}=-R^{2} .
\end{aligned}
$$

Clearly, the $\mu_{k}, u_{k}, \lambda_{k}$ do not depend on $\beta_{n-1}$ if $k<n-1$. Since the right-hand sides of (7.6), (7.7) are constants, we find from

$$
\frac{\partial}{\partial \beta_{n-1}} F^{1 n}(r, \theta)=\left.\frac{1}{n !} \frac{\partial}{\partial \beta_{n-1}} \frac{\partial^{n}}{\partial \varepsilon^{n}}\right|_{\varepsilon=0}(\text { the right-hand side of (7.2)) }
$$

that the only nonzero contribution comes from the first term

$$
\left(-\frac{f_{\theta \theta}}{R+f}+\frac{f_{\theta \theta}}{R}\right) \frac{1}{r} \frac{\partial \mu_{0}}{\partial r}=\frac{f_{\theta \theta} f}{R(R+f)} \frac{1}{r} \frac{\partial \mu_{0}}{\partial r} .
$$

Hence

$$
\begin{aligned}
\frac{\partial}{\partial \beta_{n-1}} F^{1 n}(r, \theta) & =\frac{\lambda_{1}^{\prime \prime}(\theta)}{R^{2}} \frac{1}{r} \frac{\partial \mu_{0}}{\partial r} \frac{\partial \lambda_{n-1}(\theta)}{\partial \beta_{n-1}} \\
& =-\lambda_{1}^{\prime \prime}(\theta) \frac{1}{r^{2}}=\lambda_{1}(\theta) \frac{l^{2}}{r^{2}} \quad \text { for } \quad r>1
\end{aligned}
$$


Similarly,

$$
\begin{aligned}
& \frac{\partial}{\partial \beta_{n-1}}\left(F^{1 n}(r, \theta)+F^{2 n}(r, \theta)\right) \\
&\left.\quad=\left.\frac{1}{n !} \frac{\partial}{\partial \beta_{n-1}} \frac{\partial^{n}}{\partial \varepsilon^{n}}\right|_{\varepsilon=0} \text { (the right-hand side of }(7.3)\right)=0 \quad \text { for } \quad r<1 .
\end{aligned}
$$

A direct computation shows that

$$
\begin{aligned}
-\frac{\partial}{\partial \beta_{n-1}} F^{3 n}(\theta) & =\left.\frac{1}{n !} \frac{\partial}{\partial \beta_{n-1}} \frac{\partial^{n}}{\partial \varepsilon^{n}}\right|_{\varepsilon=0} \text { (the right-hand side of (7.4)) } \\
& =\lambda_{1}(\theta)+\beta_{1} \frac{\partial}{\partial \beta_{n-1}} \Lambda_{0}^{n-1}=\lambda_{1}(\theta)-R^{2} \beta_{1}
\end{aligned}
$$

Finally, for $r<1$,

$$
\begin{aligned}
\frac{\partial}{\partial \beta_{n-1}} F^{1 n}(r, \theta)= & \left.\frac{1}{n !} \frac{\partial}{\partial \beta_{n-1}} \frac{\partial^{n}}{\partial \varepsilon^{n}}\right|_{\varepsilon=0} \text { (the right-hand side of (7.2)) } \\
= & \frac{\lambda_{1}^{\prime \prime}(\theta)}{R^{2}} \frac{1}{r} \frac{\partial \mu_{0}}{\partial r} \frac{\partial \lambda_{n-1}(\theta)}{\partial \beta_{n-1}}-\frac{\lambda_{1}^{\prime \prime}(\theta)}{R} \frac{1}{r} \frac{\partial}{\partial \beta_{n-1}} \frac{\partial \mu_{n-1}}{\partial r} \\
& -\left[2 R \lambda_{1}(\theta) \frac{\partial \mu_{n-1}}{\partial \beta_{n-1}}+2 R \mu_{1}(r, \theta) \frac{\partial \lambda_{n-1}(\theta)}{\partial \beta_{n-1}}+2 \lambda_{1}(\theta) \mu_{0} \frac{\partial \lambda_{n-1}(\theta)}{\partial \beta_{n-1}}\right]
\end{aligned}
$$

where the expression in brackets comes from the coefficient of $\chi_{\{r<1\}}$ in (7.2). Using (7.6), (7.8) we get

$$
\begin{aligned}
\frac{\partial}{\partial \beta_{n-1}} & F^{1 n}(r, \theta) \\
= & -\lambda_{1}^{\prime \prime}(\theta) \frac{1}{r} \frac{\partial \mu_{0}}{\partial r}-\lambda_{1}^{\prime \prime}(\theta) \frac{1}{r} \frac{\partial}{\partial r}\left[\frac{I_{0}(R) I_{0}(R r)}{I_{1}^{2}(R)}-\frac{r}{I_{1}(R)} I_{1}(R r)\right] \\
& -2 R \lambda_{1}(\theta)\left[\frac{R I_{0}(R) I_{0}(R r)}{I_{1}^{2}(R)}-\frac{R r}{I_{1}(R)} I_{1}(R r)\right]+2 R^{3} \mu_{1}(r, \theta)+2 R^{2} \mu_{0}(r) \lambda_{1}(\theta) . \\
= & \lambda_{1}(\theta)\left\{\frac{l^{2} I_{1}(R r)}{r I_{1}(R)}+\frac{l^{2}}{r}\left[\frac{R I_{0}(R) I_{1}(R r)}{I_{1}^{2}(R)}-\frac{R r I_{0}(R r)}{I_{1}(R)}\right]\right. \\
& \left.-2 R\left[\frac{R I_{0}(R) I_{0}(R r)}{I_{1}^{2}(R)}-\frac{R r}{I_{1}(R)} I_{1}(R r)\right]-2 R^{3} \frac{l+1}{2 l} \frac{I_{l}(R r)}{R I_{l}(R)}+2 R^{2} \mu_{0}(r)\right\}
\end{aligned}
$$

where in the last equality we have also used (3.14). Substituting (7.9)-(7.12) into (7.1), we conclude that

$$
\begin{aligned}
\widetilde{\Lambda} & =\left(2 l-R \frac{I_{l-1}(R)}{I_{l}(R)}\right) l+\frac{2 l}{I_{l}(R)} \int_{0}^{1} r I_{l}(R r)\left\{\frac{l^{2} I_{1}(R r)}{r I_{1}(R)}+\frac{l^{2}}{r}\left[\frac{R I_{0}(R) I_{1}(R r)}{I_{1}^{2}(R)}-\frac{R r I_{0}(R r)}{I_{1}(R)}\right]\right. \\
& \left.-2 R\left[\frac{R I_{0}(R) I_{0}(R r)}{I_{1}^{2}(R)}-\frac{R r}{I_{1}(R)} I_{1}(R r)\right]-2 R^{3} \frac{l+1}{2 l} \frac{I_{l}(R r)}{R I_{l}(R)}+2 R^{2} \mu_{0}(r)\right\} \mathrm{d} r-R \frac{I_{l-1}(R)}{I_{l}(R)}
\end{aligned}
$$

which is independent of $n$. 
REMARK 7.1 As was already stated before, for the solution $\left(u_{n}, \mu_{n}, \lambda_{n}, \beta_{n-1}\right)$ constructed by using Lemmas 6.4, 7.1, the mode $l$ component is not unique, for we can add to it any multiple of $\left(\mu_{1}, u_{1}, \lambda_{1}\right)$. We shall henceforth fix this multiple uniquely by the condition

$$
\int_{0}^{2 \pi} \lambda_{n}(\theta) \cos l \theta=0 \quad \text { for } \quad n \geqslant 2 .
$$

This normalization is not necessarily the same as the normalization in Remark 4.2, because the methods of construction of the solution are different.

We shall say that a linear function space $X$ with norm $\|\cdot\|_{X}$ has the algebra property if, whenever $f, g$ belong to $X$, also $f g$ belongs to $X$ and

$$
\|f g\|_{X} \leqslant C\|f\|_{X}\|g\|_{X}
$$

where $C$ is a constant independent of $f, g$. In what follows we shall need the algebra property in a more general sense, whereby $g$ belongs to one space $X_{1}$, and $f$ belongs to another space, $X_{2}$, and

$$
\|f g\|_{X_{2}} \leqslant C\|g\|_{X_{1}}\|f\|_{X_{2}} .
$$

In order to estimate the $F^{j n}$ we need to have the algebra property for the various terms in the $F^{j n}$. The spaces $L^{2}\left(B_{1}\right)$ or $H^{1 / 2}\left(B_{1}\right)$ are not suitable for this purpose. Noticing that the functions defining $F^{j}$ in (7.2)-(7.4) have the form $g(\theta) \varphi(r, \theta)$, it is convenient to work with the norm

$$
\|\psi(r, \theta)\|_{W_{2}^{m, n}}=\sum_{0 \leqslant j \leqslant m, 0 \leqslant k \leqslant n}\left\|\partial_{r}^{j} \partial_{\theta}^{k} \psi\right\|_{L^{2}}
$$

where $m, n$ are integers; notice that this definition is a little different from the usual Sobolev norm since, for example, $1 / r^{2}$ factor is not present in the $\partial_{\theta}^{2}$ derivative. We shall need on one occasion to also use the norm

$$
\|\psi(r, \theta)\|_{W_{2}^{1,3 / 2}} \equiv\|\psi(r, \theta)\|_{W_{2}^{1,1}}+\left\|\partial_{\theta}^{1 / 2} \psi(r, \theta)\right\|_{W_{2}^{1,1}} .
$$

The following estimates are well known (see, for example, [1]).

LEMMA 7.2

$$
\begin{aligned}
& \|g(\theta) \varphi(r, \theta)\|_{W_{2}^{0, k}\left(B_{1}\right)} \leqslant\|g\|_{H^{k}\left(\partial B_{1}\right)}\|\varphi(r, \theta)\|_{W_{2}^{0, k}\left(B_{1}\right)} \quad \text { for } \quad k \geqslant 1, \\
& \|g(\theta) h(\theta)\|_{H^{s}\left(\partial B_{1}\right)} \leqslant\|g\|_{H^{s}\left(\partial B_{1}\right)}\|h\|_{H^{s}\left(\partial B_{1}\right)} \quad \text { for } \quad s>1 / 2, \\
& \|\varphi(1, \theta)\|_{H^{m+1 / 2}\left(\partial B_{1}\right)} \leqslant C\|\varphi(r, \theta)\|_{W_{2}^{1, m+1}\left(B_{1}\right)} \quad \text { for } \quad m \geqslant 0 .
\end{aligned}
$$

The first two inequalities provide the algebra properties needed to estimate the $F^{j n}$. These inequalities are also valid if $B_{1}$ is replaced by $\mathbb{R}^{2} \backslash B_{1}$.

Let $H_{0}, H, B$ and $\Gamma$ be positive constants $\geqslant 1$ and set

$$
E(n, k)=k ! \frac{H_{0} H^{n-1}}{n^{2}} \frac{B^{k}}{k^{2}} \quad \text { for } \quad n \geqslant 0, k \geqslant 0,
$$


with the following convention:

$$
H^{n-1}=1 \quad \text { if } \quad n=0, \quad n^{2}=1 \quad \text { if } \quad n=0, \quad k^{2}=1 \quad \text { if } \quad k=0 .
$$

We inductively assume that, for $1 \leqslant s<n$,

$$
\begin{aligned}
& \|\left(\left|\partial_{\theta}^{k}\left(\mu_{s}-\mu_{s}^{0}(r)\right)\right|+\left|\partial_{\theta}^{k}\left(\frac{1}{r} \partial_{r}\left(\mu_{s}-\mu_{s}^{0}(r)\right)\right)\right|\right. \\
& \left.\quad+\left|\partial_{\theta}^{k}\left(\frac{1}{r} \partial_{r} \partial_{\theta} \mu_{s}\right)\right|+\left|\partial_{\theta}^{k} \partial_{r}^{2}\left(\mu_{s}-\mu_{s}^{0}(r)\right)\right|\right) \|_{W_{2}^{0,1}\left(B_{1}\right)} \leqslant E(s, k), \\
& \left\|r^{2}\left(\left|\partial_{\theta}^{k} \mu_{s}\right|+\left|\partial_{\theta}^{k}\left(\frac{1}{r} \partial_{r} \mu_{s}\right)\right|+\left|\partial_{\theta}^{k}\left(\frac{1}{r} \partial_{r} \partial_{\theta} \mu_{s}\right)\right|+\left|\partial_{\theta}^{k} \partial_{r}^{2} \mu_{s}\right|\right)\right\|_{W_{2}^{0,1}\left(\mathbb{R}^{2} \backslash B_{1}\right)} \leqslant E(s, k), \\
& \|\left(\left|\partial_{\theta}^{k}\left(\mu_{s}+u_{s}\right)\right|+\left|\partial_{\theta}^{k}\left(\frac{1}{r} \partial_{r}\left(\mu_{s}+u_{s}\right)\right)\right|\right. \\
& \left.\quad+\left|\partial_{\theta}^{k}\left(\frac{1}{r} \partial_{r} \partial_{\theta}\left(\mu_{s}+u_{s}\right)\right)\right|+\left|\partial_{\theta}^{k} \partial_{r}^{2}\left(\mu_{s}+u_{s}\right)\right|\right) \|_{W_{2}^{0,1}\left(B_{1}\right)} \leqslant E(s, k), \\
& \left\|\partial_{\theta}^{k}\left(\lambda_{s}+\beta_{s} R^{2}\right)\right\|_{H^{5 / 2}\left(\partial B_{1}\right)} \leqslant E(s, k), \\
& \left|\beta_{s}\right| \leqslant \Gamma E(s, 0)
\end{aligned}
$$

where $\mu_{n}^{0}(r)$ is defined in (6.16)-(6.18) with $\lambda_{s}^{0}=\Lambda_{0}=\frac{1}{2 \pi} \int_{0}^{2 \pi} \lambda_{s}(\theta) \mathrm{d} \theta$ in (6.17), and $C_{20}^{s}=C_{20}$ in (6.18) is defined by (6.36)-(6.39) with $F^{j} \equiv F^{j s}$ :

$$
\mu_{s}^{0}=\frac{\lambda_{s}^{0}}{R}\left[\frac{r I_{1}(R r)}{I_{1}(R)}-\frac{I_{0}(R r)}{I_{0}(R)}\right]+C_{20}^{s} \frac{I_{0}(R r)}{I_{0}(R)}
$$

Note that (7.23) implies that

$$
\left\|\partial_{\theta}^{k} \partial_{\theta}^{3 / 2}\left(\lambda_{s}+\beta_{s} R^{2}\right)\right\|_{W_{2}^{0,1}\left(B_{1}\right)} \leqslant C E(s, k) .
$$

For $s=1,2$, the solutions $\left(\mu_{s}, u_{s}, \lambda_{s}\right)$ are given in terms of the explicit analytic functions and the estimates (7.19)-(7.23) are valid if we choose $H_{0}$ to be large enough and $H \geqslant 1, \Gamma \geqslant 1$.

We proceed to establish the estimates (7.19)-(7.23) for $s=n$ assuming $n \geqslant 3$ and $H, \Gamma$ large enough (independent of $n$ ).

In proving these estimates we shall use several lemmas which deal with estimating derivatives of composite functions; these lemmas are stated and proved in Appendix B.

Step 1. Estimating $F^{j, n}$. 
We need to estimate $F^{j, n}$ for $j=1,2,3$. To estimate $F^{3, n}$, we rewrite (7.4) as

$$
\begin{aligned}
-\sum_{m} \geqslant 1 & \varepsilon^{m} F^{3 m} \\
& =\left(\sum_{m \geqslant 0} \varepsilon^{m} \beta_{m}\right)\left[\frac{(R+f)^{2}}{\left[(R+f)^{2}+f_{\theta}^{2}\right]^{1 / 2}}-R\right]-\frac{1}{R} f \\
& =\left(\sum_{m \geqslant 2} \varepsilon^{m} \beta_{m}\right)\left[\frac{(R+f)^{2}}{\left[(R+f)^{2}+f_{\theta}^{2}\right]^{1 / 2}}-R\right]+\frac{1}{R}\left[\frac{(R+f)^{2}}{\left[(R+f)^{2}+f_{\theta}^{2}\right]^{1 / 2}}-R-f\right] \\
& \equiv \sum \varepsilon^{m} \mathcal{Q}_{1, m}+\sum \varepsilon^{m} \mathcal{Q}_{2, m} .
\end{aligned}
$$

We can write

$$
\frac{(R+\xi)^{2}}{\left[(R+\xi)^{2}+\zeta^{2}\right]^{1 / 2}}-R-\xi=-\frac{1}{2 R} \zeta^{2}+\zeta^{2} \sum_{1 \leqslant i+j<\infty} a_{i j} \xi^{i} \zeta^{2 j},
$$

where $a_{i j}$ are constants such that

$$
\left|a_{i j}\right| \leqslant A_{0} A^{i+j} .
$$

Note that $\left[\frac{(R+f)^{2}}{\left[(R+f)^{2}+f_{\theta}^{2}\right]^{1 / 2}}-R\right]$ starts with $\varepsilon$ order terms and $\left[\frac{(R+f)^{2}}{\left[(R+f)^{2}+f_{\theta}^{2}\right]^{1 / 2}}-R-f\right]$ starts with $\varepsilon^{2}$ order terms. Since the terms involving orders $>n$ in the right-hand sides do not appear among the terms of $F^{j, n}$, we may replace them by 0 when estimating $F^{j, n}$. By (7.23), $\left|\beta_{n-1}\right| \leqslant E(n-1,0) \leqslant$ $\frac{\Gamma}{H} E(n, 0)$. Using also (7.23) and applying (B.11) of Lemma B.4 and Lemma B.1 to the $\mathcal{Q}_{1, m}$ terms, we obtain

$$
\left\|\partial_{\theta}^{k} \mathcal{Q}_{1, n}\right\|_{H^{3 / 2}\left(\partial B_{1}\right)} \leqslant \frac{C(\Gamma)}{H} E(n, k) .
$$

Using (7.22) and applying (B.12) of Lemma B.4 to the $\mathcal{Q}_{2 m}$ terms, we also get

$$
\left\|\partial_{\theta}^{k} \mathcal{Q}_{2, n}\right\|_{H^{3 / 2}\left(\partial B_{1}\right)} \leqslant \frac{C(\Gamma)}{H} E(n, k),
$$

so that

$$
\left\|\partial_{\theta}^{k} F^{3, n}\right\|_{H^{3 / 2}\left(\partial B_{1}\right)} \leqslant \frac{C(\Gamma)}{H} E(n, k) .
$$

We next estimate the terms in (7.2) and (7.3) contributing to $F^{1, n}$ and $F^{1, n}+F^{2, n}$. For $r>1$, we can write

$$
\begin{aligned}
\left(-\frac{f_{\theta \theta}}{R+f}+\frac{f_{\theta \theta}}{R}\right) \frac{1}{r} \frac{\partial \mu_{0}}{\partial r}+\frac{f_{\theta}^{2}}{(R+f)^{2}}\left(\frac{\partial^{2} \mu_{0}}{\partial r^{2}}+\frac{2}{r} \frac{\partial \mu_{0}}{\partial r}\right) & =\left[\left(-\frac{f_{\theta}}{R+f}+\frac{f_{\theta}}{R}\right) \frac{1}{r^{2}}\right]_{\theta} \\
& \equiv \sum \varepsilon^{m} \frac{1}{r^{2}} \frac{\partial}{\partial \theta} g_{m}(\theta) ;
\end{aligned}
$$

note that $\left(-\frac{f_{\theta}}{R+f}+\frac{f_{\theta}}{R}\right)$ starts with $\varepsilon^{2}$ order terms. Applying (B.12) of Lemma B.4, we obtain

$$
\left\|\partial_{\theta}^{k} g_{n}\right\|_{H^{3 / 2}\left(\partial B_{1}\right)} \leqslant \frac{C(\Gamma)}{H} E(n, k) .
$$


Similarly,

$$
\begin{aligned}
&- \frac{f_{\theta \theta}}{R+f} \sum_{n \geqslant 1} \varepsilon^{n} \frac{1}{r} \frac{\partial \mu_{n}}{\partial r}-\frac{2 f_{\theta}}{R+f} \sum_{n \geqslant 1} \varepsilon^{n} \frac{1}{r} \frac{\partial^{2} \mu_{n}}{\partial \theta \partial r}+\frac{f_{\theta}^{2}}{(R+f)^{2}} \sum_{n \geqslant 1} \varepsilon^{n}\left(\frac{\partial^{2} \mu_{n}}{\partial r^{2}}+\frac{2}{r} \frac{\partial \mu_{n}}{\partial r}\right) \\
&=-\left[\frac{f_{\theta}}{R+f} \sum_{n \geqslant 1} \varepsilon^{n} \frac{1}{r} \frac{\partial \mu_{n}}{\partial r}\right]_{\theta}+\left\{\frac{f_{\theta}^{2}}{(R+f)^{2}} \sum_{n \geqslant 1} \varepsilon^{n}\left(\frac{\partial^{2} \mu_{n}}{\partial r^{2}}+\frac{1}{r} \frac{\partial \mu_{n}}{\partial r}\right)-\frac{f_{\theta}}{R+f} \sum_{n \geqslant 1} \varepsilon^{n} \frac{1}{r} \frac{\partial^{2} \mu_{n}}{\partial \theta \partial r}\right\} \\
& \equiv \sum_{m} \varepsilon^{m} \frac{\partial}{\partial \theta} G_{m}^{1}(r, \theta)+\sum_{m} \varepsilon^{m} K_{m}(r, \theta) .
\end{aligned}
$$

Using (7.20), (7.22) and Lemmas B.1, B.4, we obtain

$$
\begin{aligned}
& \left\|r^{3} \partial_{\theta}^{k} \partial_{r} G_{n}^{1}\right\|_{W_{2}^{0,1}\left(\mathbb{R}^{2} \backslash B_{1}\right)}+\left\|r^{2} \partial_{\theta}^{k} G_{n}^{1}\right\|_{W_{2}^{1,3 / 2}\left(\mathbb{R}^{2} \backslash B_{1}\right)} \leqslant \frac{C(\Gamma)}{H} E(n, k) \\
& \left\|r^{2} \partial_{\theta}^{k} K_{n}\right\|_{W_{2}^{0,1}\left(\mathbb{R}^{2} \backslash B_{1}\right)} \leqslant \frac{C(\Gamma)}{H} E(n, k) .
\end{aligned}
$$

This completes the estimate for $F^{1, n}$ in the region $r>1$. The estimate for $F^{1, n}$ and $F^{1, n}+F^{2, n}$ in the region $r<1$ can be carried out in a similar way. Using the equations of $\mu_{n}, u_{n}, \lambda_{n}$ and the equations obtained by differentiation $k+1$ times in $\theta$, we can apply Lemma 6.4 and Remark 6.4 to conclude that

$$
\text { [the left-hand sides of (7.19)-(7.22) for } s=n] \leqslant \frac{C(\Gamma)}{H} E(n, k) \quad \text { for } \quad k \geqslant 0 \text {. }
$$

Note that the part of Lemma 6.4 which is based on Lemma 6.2 is used in handling $\frac{\partial}{\partial \theta} g_{n}, \frac{\partial}{\partial \theta} G_{n}^{1}$ and similar terms from $F^{1, n}$ in $\{r>1\}$ as well as from $F^{1, n}$ in $\{r<1\}$, whereas the part based on Lemma 6.1 is used in handling $K_{n}$ and similar terms from $F^{1 n}$ in $\{r>1\}$.

Step 2. Estimating $\beta_{n}$.

Recall that $\beta_{n}$ is determined by equation (6.85) with $F_{l}^{j}=F_{l}^{j, n+1}$ for $j=1,2,3$ (only mode $l$ terms enter into the compatibility condition).

The coefficient for $\beta_{n}$ in (6.85) is given by (7.13), which is independent of $n$ and $\neq 0$. Consequently, $\beta_{n}$ is estimated by those terms in $F_{l}^{j, n+1}$ which do not depend on $\beta_{n}$. We denote these terms by $\widetilde{F}_{l}^{j, n+1}$, i.e. $\widetilde{F}_{l}^{j, n+1}=\left.F_{l}^{j, n+1}\right|_{\beta_{n}=0}$. We need to estimate these terms and we begin with

(a) $\widetilde{F}_{l}^{3, n+1}$. 
By (7.25),

$$
\begin{aligned}
- & \left.\sum_{m \geqslant 1} \varepsilon^{m} F^{3 m}\right|_{\beta_{n}=0} \\
= & {\left[\left(\sum_{m \geqslant 2} \varepsilon^{m} \beta_{m}\right) f-\left(\sum_{m \geqslant 2} \varepsilon^{m} \beta_{m}\right) \frac{f_{\theta}^{2}}{2 R}+\left(\sum_{m \geqslant 2} \varepsilon^{m} \beta_{m}\right) f_{\theta}^{2} \sum_{1 \leqslant i+j<\infty} a_{i j} \xi^{i}\left(f_{\theta}\right)^{2 j}\right.} \\
& \left.\quad-\frac{1}{2 R^{2}} f_{\theta}^{2}+\frac{f_{\theta}^{2}}{R} \sum_{1 \leqslant i+j<\infty} a_{i j} \xi^{i}\left(f_{\theta}\right)^{2 j}\right]_{\beta_{n}=0} \\
\equiv & {\left[\sum \varepsilon^{m} \mathcal{P}_{1, m}+\sum \varepsilon^{m} \mathcal{P}_{2, m}+\sum \varepsilon^{m} \mathcal{P}_{3, m}+\sum \varepsilon^{m} \mathcal{P}_{4, m}+\sum \varepsilon^{m} \mathcal{P}_{5, m}\right]_{\beta_{n}=0} . }
\end{aligned}
$$

Clearly, the second, third, and the fifth sum are products of at least three series. Thus, by Lemmas B.1 and B.4, using also the embedding inequality $\|g\|_{L^{\infty}} \leqslant C\|g\|_{H^{s}}$ for $s>1 / 2$,

$$
\left[\left|\mathcal{P}_{2, n+1}\right|+\left|\mathcal{P}_{3, n+1}\right|+\left|\mathcal{P}_{5, n+1}\right|\right]_{\beta_{n}=0} \leqslant \frac{C(\Gamma)}{H^{2}} E(n+1,0) \leqslant \frac{C(\Gamma)}{H} E(n, 0) .
$$

Next, by Remark 7.1, the $\lambda_{m}(\theta)$ for $m \geqslant 2$ do not contain mode $l$ terms. Therefore, the only term in $\sum \varepsilon^{m} \mathcal{P}_{1, m}$ of order $\varepsilon^{n+1}$ and of mode $l$ is given by $\beta_{n} \lambda_{1}(\theta)$; hence, $\left|\mathcal{P}_{1, n+1}\right|_{\beta_{n}=0}=0$.

Finally,

$$
\mathcal{P}_{4, n+1}=-\frac{1}{2 R^{2}} \sum_{k=2}^{n-1} \partial_{\theta} \lambda_{n+1-k} \partial_{\theta} \lambda_{k},
$$

so that, by the inductive assumptions and Lemma B.1,

$$
\left|P_{4, n+1}\right| \leqslant \frac{C}{H} E(n+1,0) \leqslant C E(n, 0) .
$$

Combining these estimates, we conclude that

$$
\left|\widetilde{F}_{l}^{3, n+1}+\beta_{n}\right| \leqslant\left[C+\frac{C(\Gamma)}{H}\right] E(n, 0) .
$$

(b) $\widetilde{F}_{l}^{1, n+1}+\widetilde{F}_{l}^{2, n+1}$.

Clearly, $\widetilde{F}_{l}^{1, n+1}+\widetilde{F}_{l}^{2, n+1}=\frac{1}{\pi} \int_{0}^{2 \pi}\left(\widetilde{F}^{1, n+1}+\widetilde{F}^{2, n+1}\right) \cos l \theta \mathrm{d} \theta$.

To estimate the contribution from the first term in the right-hand side of (7.3) we multiply by $\cos l \theta$ and integrate over $\theta, 0 \leqslant \theta \leqslant 2 \pi$; in this way we obtain the coefficients of mode $l$ terms. Next we integrate by parts to reduce the derivative $\partial_{\theta}^{2} f$. We get

$$
\begin{aligned}
&-\int_{0}^{2 \pi} \frac{2 f_{\theta}}{R+f} \sum_{m \geqslant 1} \varepsilon^{m} \frac{1}{r} \frac{\partial^{2}\left(\mu_{m}+u_{m}\right)}{\partial \theta \partial r} \cos l \theta \mathrm{d} \theta \\
&+\int_{0}^{2 \pi} \frac{2 f_{\theta}^{2}}{(R+f)^{2}} \sum_{m \geqslant 1} \varepsilon^{m} \frac{1}{r} \frac{\partial\left(\mu_{m}+u_{m}\right)}{\partial r} \cos l \theta \mathrm{d} \theta \\
&-\int_{0}^{2 \pi} \frac{f_{\theta}}{R+f} \sum_{m \geqslant 1} \varepsilon^{m} \frac{1}{r} \frac{\partial\left(\mu_{m}+u_{m}\right)}{\partial r} \cos l \theta \mathrm{d} \theta \\
& \equiv-J_{1}+J_{2}-J_{3} .
\end{aligned}
$$


We also expand

$$
\frac{1}{R+f}=\frac{1}{R}-\frac{1}{R} \sum_{m \geqslant 1} \frac{(-f)^{m}}{R^{m}} .
$$

Now recall that $f_{\theta}$ is independent of $\Gamma$ (but $f$ depends on $\Gamma$ ). As we substitute the expansion of $1 /(R+f)$ into the $J_{k}$, we observe that whenever a factor $f^{k}$ appears in a particular term, it comes in a product of at least three series in $\varepsilon$, each with no zero-order term. We can therefore apply Lemma B.1 and the inductive assumptions to get, at $\beta_{n}=0$, the bound

$$
C_{0}\left(\frac{C_{0}}{H}\right)^{k-1} \frac{C(\Gamma)}{H} E(n, 0)
$$

where $C_{0}$ depends only on $H_{0}$. There remain only the terms which do not depend on $f$ and they come as product of two series. Using the inductive assumption we can bound them, at $\beta_{n}=0$, by $C E(n, 0)$. We thus conclude that the coefficient of mode $l$ in $\varepsilon^{n+1}$ of the first term in the right-hand side of (7.3) is bounded, at $\beta_{n}=0$, by

$$
\left[C+\frac{C(\Gamma)}{H}\right] E(n, 0)
$$

The other terms in the right-hand side of (7.3) can be estimated in a similar manner. Thus

$$
\int_{0}^{1} \tau^{l+1}\left|\widetilde{F}_{l}^{1, n+1}(\tau)+\widetilde{F}_{l}^{2, n+1}(\tau)\right| \mathrm{d} \tau \leqslant\left[C+\frac{C(\Gamma)}{H}\right] E(n, 0) .
$$

(c) $\widetilde{F}_{l}^{1, n+1}(r), r>1$.

$\widetilde{F}_{l}^{1, n+1}$ in the region $r>1$ can be handled as in (b), except for the first two terms in (7.2), which give

$$
\begin{aligned}
\frac{1}{\pi} \int_{0}^{2 \pi}\left[\left(-\frac{f_{\theta}}{R+f}+\frac{f_{\theta}}{R}\right) \frac{1}{r^{2}}\right]_{\theta} \cos l \theta \mathrm{d} \theta & =\frac{l}{\pi} \int_{0}^{2 \pi}\left[\left(-\frac{f_{\theta}}{R+f}+\frac{f_{\theta}}{R}\right) \frac{1}{r^{2}}\right] \sin l \theta \mathrm{d} \theta \\
& =\frac{l}{\pi R r^{2}} \int_{0}^{2 \pi} \frac{f_{\theta} f}{f+R} \sin l \theta \mathrm{d} \theta .
\end{aligned}
$$

We write $f_{\theta} f /(f+R)$ in the form $f_{\theta} f / R+\cdots$, where the '. ' ' refers to terms which are products of at least three series for which we can apply Lemma B.4 to get an extra $1 / H$ factor. The remaining term is

$$
\frac{l}{\pi R r^{2}} \int_{0}^{2 \pi} \frac{f_{\theta} f}{R} \sin l \theta \mathrm{d} \theta=\frac{l}{\pi R r^{2}} \int_{0}^{2 \pi} \frac{f_{\theta}\left(f-f_{0}\right)}{R} \sin l \theta \mathrm{d} \theta+\frac{l f_{0}}{\pi R r^{2}} \int_{0}^{2 \pi} \frac{f_{\theta}}{R} \sin l \theta \mathrm{d} \theta,
$$

where $f_{0}=\frac{1}{2 \pi} \int_{0}^{2 \pi} f(\theta) \mathrm{d} \theta$. Since $f_{\theta}$ is independent of $\Gamma$ and $f-f_{0}$ can be estimated in the $L^{2}$ norm by the $\theta$ derivatives of $f$, the coefficient of $\varepsilon^{n+1} \cos l \theta$ in the first term in the right-hand side of (7.39), at $\beta_{n}=0$, is bounded by

$$
\frac{C}{H} E(n+1,0)=C E(n, 0) .
$$


To estimate the coefficient of $\varepsilon^{n+1} \cos l \theta$ in the second term, note that by (7.14),

$$
\int_{0}^{2 \pi} f_{\theta} \sin l \theta \mathrm{d} \theta=\varepsilon \int_{0}^{2 \pi} \lambda_{1}^{\prime}(\theta) \sin l \theta \mathrm{d} \theta=-\pi l \varepsilon
$$

It follows that this coefficient is equal to

$$
-\frac{l^{2}}{R^{2} r^{2}} \lambda_{n 0}
$$

where $\lambda_{n 0}$ is the zero mode of $\lambda_{n}$ and, by (7.22), $\left|\lambda_{n 0}\right| \leqslant E(n, 0)$ if $\beta_{n}=0$. Thus, the second term on the right-hand side of (7.39) does not contribute anything to $\widetilde{F}_{l}^{1, n+1}$. In summary,

$$
\int_{1}^{\infty} \tau^{-l+1}\left|\widetilde{F}_{l}^{1, n+1}(\tau)\right| \mathrm{d} \tau \leqslant\left[C+\frac{C(\Gamma)}{H}\right] E(n, 0) .
$$

(d) $\widetilde{F}_{l}^{1, n+1}(r), r<1$.

The estimate for $\widetilde{F}_{l}^{1, n+1}$ in $r<1$ is the most difficult, since some of the derivatives of $\mu_{n}$ involve $\beta_{n}$. The terms that arise as the product of three series are treated in a same way as before. The remaining terms come from

$$
\begin{aligned}
\frac{f_{\theta \theta} f}{R^{2}} \frac{1}{r} & \frac{\partial \mu_{0}}{\partial r}+\frac{f_{\theta}^{2}}{R^{2}}\left(\frac{\partial^{2} \mu_{0}}{\partial r^{2}}+\frac{2}{r} \frac{\partial \mu_{0}}{\partial r}\right)-\frac{2 f_{\theta}}{R} \sum_{n \geqslant 1} \varepsilon^{n} \frac{1}{r} \frac{\partial^{2} \mu_{n}}{\partial \theta \partial r} \\
& -\frac{f_{\theta \theta}}{R} \sum_{n \geqslant 1} \varepsilon^{n} \frac{1}{r} \frac{\partial \mu_{n}}{\partial r}-2 R f \sum_{n \geqslant 1} \varepsilon^{n} \mu_{n}-f^{2} \mu_{0} \\
= & \left(\frac{f_{\theta} f}{R^{2}}\right)_{\theta} \frac{1}{r} \frac{\partial \mu_{0}}{\partial r}+\frac{f_{\theta}^{2}}{R^{2}}\left(\frac{\partial^{2} \mu_{0}}{\partial r^{2}}+\frac{1}{r} \frac{\partial \mu_{0}}{\partial r}\right)-\frac{f_{\theta}}{R} \sum_{n \geqslant 1} \varepsilon^{n} \frac{1}{r} \frac{\partial^{2} \mu_{n}}{\partial \theta \partial r} \\
& -\left(\frac{f_{\theta}}{R} \sum_{n \geqslant 1} \varepsilon^{n} \frac{1}{r} \frac{\partial \mu_{n}}{\partial r}\right)_{\theta}-2 R f \sum_{n \geqslant 1} \varepsilon^{n} \mu_{n}-f^{2} \mu_{0} \\
\equiv & \sum \varepsilon^{m} \mathcal{R}_{1, m}+\sum \varepsilon^{m} \mathcal{R}_{2, m}+\sum \varepsilon^{m} \mathcal{R}_{3, m}+\sum \varepsilon^{m} \mathcal{R}_{4, m}+\sum \varepsilon^{m} \mathcal{R}_{5, m}+\sum \varepsilon^{m} \mathcal{R}_{6, m} .
\end{aligned}
$$

The $\varepsilon^{n+1}$ terms in the series $\sum \varepsilon^{m} \mathcal{R}_{2, m}$ and $\sum \varepsilon^{m} \mathcal{R}_{3, m}$ are estimated by the inductive assumptions independently of $\Gamma$. Thus, as before,

$$
\left[\left\|\mathcal{R}_{2, n+1}\right\|+\left\|\mathcal{R}_{3, n+1}\right\|\right]_{\beta_{n}=0} \leqslant \operatorname{CE}(n, 0),
$$

where the norm $\|\cdot\|$ is defined in (7.37). The series $\sum \varepsilon^{m} \mathcal{R}_{1, m}$ can be treated in a same way as in part (c):

$$
\begin{aligned}
\frac{1}{\pi} \int_{0}^{2 \pi} & \left(\frac{f_{\theta} f}{R^{2}}\right)_{\theta} \frac{1}{r} \frac{\partial \mu_{0}}{\partial r} \cos l \theta \mathrm{d} \theta \\
& =\frac{l}{R^{2} \pi} \frac{1}{r} \frac{\partial \mu_{0}}{\partial r} \int_{0}^{2 \pi} f_{\theta}(\theta)\left[f(\theta)-f_{0}\right] \sin l \theta \mathrm{d} \theta+\frac{l f_{0}}{R^{2} \pi} \frac{1}{r} \frac{\partial \mu_{0}}{\partial r} \int_{0}^{2 \pi} f_{\theta} \sin l \theta \mathrm{d} \theta,
\end{aligned}
$$


where the estimate for the first term is independent of $\Gamma$ whereas the coefficient of $\varepsilon^{n+1} \cos l \theta$ from the second term is zero when we set $\beta_{n}=0$.

To estimate the coefficient of mode $l$ in $\left.\mathcal{R}_{4, n+1}\right|_{\beta_{n}=0}$ we write

$$
\mu_{m}=\left[\mu_{m}(r, \theta)-\mu_{m}^{0}(r)\right]+\mu_{m}^{0}(r) .
$$

Then the series corresponding to $\mu_{m}(r, \theta)-\mu_{m}^{0}(r)$ (in $\left.\sum \mathcal{R}_{4, m}\right)$ can be treated as before, as product of two series, using Lemma B.1 and inductive assumption; since $\partial_{r}\left(\mu_{m}-\mu_{m}^{0}\right)$ is independent of $\Gamma$, we get the bound $C E(n, 0)$. Thus, it remains to estimate the coefficient of $\varepsilon^{n+1} \cos l \theta$ in

$$
-\left(\frac{f_{\theta}}{R}\right)_{\theta} \sum_{m \geqslant 1} \varepsilon^{m} \frac{1}{r} \frac{\partial \mu_{m}^{0}(r)}{\partial r} \quad \text { when } \quad \beta_{n}=0,
$$

where $\mu_{m}^{0}(r)$ is defined as in (7.24) and the constant $C_{20}^{m}$ is estimated by (6.14) with $\Lambda=\lambda_{m}, M=$ $\mu_{m}, U=u_{m}, \beta=\beta_{m}$. Again, since $f-\varepsilon \lambda_{1}(\theta)$ has no mode $l$ terms, the $l$ mode in the coefficient of $\varepsilon^{n}$ of the above series is

$$
-\frac{1}{\pi} \int_{0}^{2 \pi}\left(\frac{\lambda_{1}^{\prime}(\theta)}{R}\right)^{\prime} \frac{1}{r} \frac{\partial \mu_{n}^{0}(r)}{\partial r} \cos l \theta \mathrm{d} \theta=l^{2} \frac{1}{R r} \frac{\partial \mu_{n}^{0}(r)}{\partial r}
$$

and, at $\beta_{n}=0$, this yields

$$
\frac{l^{2}}{R r} \frac{\lambda_{n 0}}{R} \frac{\partial}{\partial r}\left[\frac{r I_{1}(R r)}{I_{1}(R)}-\frac{I_{0}(R r)}{I_{0}(R)}\right]+C_{20}^{n} \frac{\partial}{\partial r} \frac{I_{0}(R r)}{I_{0}(R)}
$$

where $\lambda_{n 0}$ (the zero mode of $\lambda_{n}$ ) and $C_{20}^{n}$ are bounded, at $\beta_{n}=0$, by $C E(n, 0)$ (by Lemma 6.1). We conclude that

$$
\left|\int_{0}^{2 \pi} \mathcal{R}_{4, n+1} \cos l \theta \mathrm{d} \theta\right|_{\beta_{n}=0} \leqslant C E(n, 0) .
$$

Consider next $\sum \varepsilon^{m} \mathcal{R}_{5, m}$. If we decompose $\mu_{m}$ as in (7.41), then the part corresponding to $\mu_{m}-\mu_{m}^{0}$ can be treated similarly to (7.39) (with $f_{\theta}$ replaced by $\mu_{m}-\mu_{m}^{0}$ ). Thus, it remains to estimate the coefficient of $\varepsilon^{n+1} \cos l \theta$ in

$$
-2 R f \sum \varepsilon^{m} \mu_{m}^{0} \quad \text { at } \quad \beta_{n}=0
$$

which is equal to $-2 R \mu_{n}^{0}$ at $\beta_{n}=0$. As before, if $\beta_{n}=0$, then

$$
\left|\mu_{n}^{0}\right| \leqslant C\left(\left|\lambda_{n 0}\right|+\left|C_{20}^{n}\right|\right) \leqslant C E(n, 0),
$$

so that

$$
\left|\int_{0}^{2 \pi} \mathcal{R}_{5, n+1} \cos l \theta \mathrm{d} \theta\right| \leqslant C E(n, 0)
$$

Consider, finally, $\sum \varepsilon^{m} \mathcal{R}_{6, m}$. Writing

$$
f^{2}=\left(f-f_{0}\right)^{2}+2\left(f-f_{0}\right) f_{0}+f_{0}^{2},
$$


the terms $\left(f-f_{0}\right)^{2}$ and $\left(f-f_{0}\right) f_{0}$ can be treated as before and we obtain the bound $C E(n, 0)$ on the coefficient of $\varepsilon^{n+1} \cos l \theta$. Since $f_{0}^{2}$ is a constant, it has no mode $l$ terms and thus, altogether,

$$
\left|\int_{0}^{2 \pi} \mathcal{R}_{6, n+1} \cos l \theta \mathrm{d} \theta\right|_{\beta_{n}=0} \leqslant C E(n, 0) .
$$

Combining all these estimates, the compatibility condition for $\beta_{n}$ yields

$$
\left|\widetilde{\Lambda} \beta_{n}\right| \leqslant\left[C+\frac{C(\Gamma)}{H}\right] E(n, 0),
$$

where $\tilde{\Lambda}$ was defined in Lemma 7.1, and $|\tilde{\Lambda}| \geqslant c>0$.

Choosing $\Gamma$ and $H$ such that

$$
\frac{C(\Gamma)}{H} \leqslant 1, \quad \max \left\{1, \frac{1}{c}\right\}(C+1) \leqslant \Gamma
$$

(for the $C(\Gamma)$ that appear in both (7.42) and (7.32)) we conclude that (7.23) holds for $s=n$ and then, by (7.32), also (7.19)-(7.22) hold for $s=n$.

This completes the induction proof.

REMARK 7.2 Having proved (7.19)-(7.22) for all $s$, we get (with $\Gamma$ now fixed) $\left|\lambda_{s}^{0}\right| \leqslant C E(s, 0)$ (from (7.22), (7.23)) and $\left|C_{20}^{s}\right| \leqslant C E(s, 0)$ (by (6.47) applied with $F^{j}=F^{j, s}, \beta=\beta_{s}, C_{20}=$ $\left.C_{20}^{s}\right)$. Then also $\left|\mu_{s}^{0}\right| \leqslant C E(s, 0)$, by (7.25). Consequently (7.19)-(7.22) are valid after deleting the $\mu_{n}^{0}$ and $\beta_{n}$ terms from the left-hand sides, and changing $H$ to a larger number in the right-hand sides, if necessary.

Using Remark 7.2, we conclude with the following theorem.

THEOREM 7.3 There exists a solution of (5.4)-(5.8) of the form (5.2), (5.9)-(5.12) where the series are convergent for $|\varepsilon| \leqslant \varepsilon_{0}$, for some $\varepsilon_{0}>0$, and define analytic functions in $(\theta, \varepsilon)$ for $|\varepsilon| \leqslant \varepsilon_{0}, 0 \leqslant \theta \leqslant 2 \pi$; the solution is unique under the assumption that it is even in $\theta$, it contains no mode 1 terms, and

$$
\int_{0}^{2 \pi} \lambda_{n}(\theta) \cos l \theta \mathrm{d} \theta=0(n \geqslant 2) .
$$

\section{Bifurcation branches of analytic solutions for the original problem}

The estimates (7.19)-(7.22) can be extended to include derivatives with respect to $r$ to any order, namely

$$
\begin{aligned}
& \left\|r^{m} \partial_{r}^{m} \partial_{\theta}^{k} \mu_{n}\right\|_{W_{2}^{0,1}\left(B_{1}\right)} \leqslant \frac{H_{0} H^{n-1}}{n^{2}}(k+m) ! \frac{B^{k}}{k^{2}} D_{0} D^{m}, \\
& \left\|r^{m} \partial_{r}^{m} \partial_{\theta}^{k} \mu_{n}\right\|_{W_{2}^{0,1}\left(\mathbb{R}^{n} \backslash B_{1}\right)} \leqslant \frac{H_{0} H^{n-1}}{n^{2}}(k+m) ! \frac{B^{k}}{k^{2}} D_{0} D^{m} . \\
& \left\|r^{m} \partial_{r}^{m} \partial_{\theta}^{k} u_{n}\right\|_{W_{2}^{0,1}\left(B_{1}\right)} \leqslant \frac{H_{0} H^{n-1}}{n^{2}}(k+m) ! \frac{B^{k}}{k^{2}} D_{0} D^{m} .
\end{aligned}
$$


The proof is by induction on $m$. For $m=1,2$ this was already proved in (7.19), (7.20) (if we choose $D \gg B$ ). Suppose now that (8.1) is true for all $m<j$. To prove it for $m=j$ we apply $r^{j} D_{r}^{j-2} D_{\theta}^{k}$ to equations (6.3), (6.4) with $M=\mu_{n}, U=u_{n}, \Lambda=\lambda_{n}$ and then move all the terms to the right-hand side, except for $r^{j} D_{r}^{j} D_{\theta}^{k} \mu_{n}$, to get

$$
\begin{aligned}
r^{j} D_{r}^{j} D_{\theta}^{k} \mu_{n} & =r^{j} D_{r}^{j-2} D_{\theta}^{k} F^{1 n}+\chi_{\{r<1\}} r^{j} D_{r}^{j-2} D_{\theta}^{k}\left[R^{2} \mu_{n}+2 R \lambda_{n} \mu_{0}\right] \\
& +\frac{1}{R} r^{j} D_{r}^{j-2}\left(\frac{1}{r} \frac{\partial \mu_{0}}{\partial r}\right) D_{\theta}^{k+2} \lambda_{n}-r^{j} D_{r}^{j-2} D_{\theta}^{k}\left(\frac{1}{r} D_{r} \mu_{n}\right)-r^{j} D_{r}^{j-2}\left(\frac{1}{r^{2}} D_{\theta}^{k+2} \mu_{n}\right) .
\end{aligned}
$$

From (7.2)-(7.4) we find that the terms in $F^{1 n}$ are of the form to which Lemma B.5 can be applied. Thus these terms can be estimated in the same way as in Section 7: namely, we consider $D_{\theta}^{n} F^{1 n}$ as a function to which we apply successive r-derivatives and use Lemma B.5 and the induction assumption on $j$. Similarly, other terms can be estimated using induction. We remark here that in the last term, which involves $D_{\theta}^{k+2} D_{r}^{j-2} \mu_{n}$, we 'lose' two $\theta$ derivatives, but we 'gain' two $r$ derivatives; the $(k+m)$ ! factor together with the choice $D \gg B$ enable us to carry out the induction for $\mu_{n}$, and similarly for $u_{n}$.

The estimates in (8.1) show that $\mu$ is analytic in $(x, \varepsilon)$ if $0<|x| \leqslant 1,|\varepsilon| \leqslant \varepsilon_{0}$ for some $\varepsilon_{0}>0$, and in $1 \leqslant|x|,|\varepsilon| \leqslant \varepsilon_{0}$ and $u$ is analytic in $(x, \varepsilon)$ if $0<|x| \leqslant 1,|\varepsilon| \leqslant \varepsilon_{0}$, for some $\varepsilon_{0}$; recall that the free boundary is analytic in $(\theta, \varepsilon)$ if $0 \leqslant \theta \leqslant 2 \pi,|\varepsilon| \leqslant \varepsilon_{0}$.

These facts allow us to reverse the mapping (5.1) and obtain well defined analytic functions

$$
\mu(x, \varepsilon), u(x, \varepsilon)
$$

and free boundary $r=R_{0 l}+f(\theta, \varepsilon)$ in the original variables, which satisfy the system (1.1)-(1.6) with $\beta$ as in (1.8); more precisely,

$$
\begin{aligned}
& \mu^{+}(x, \varepsilon) \text { is analytic in }(x, \varepsilon) \text { for }|x| \geqslant R_{0 l}+f(\theta, \varepsilon), \quad|\varepsilon| \leqslant \varepsilon_{0}, \\
& \mu^{-}(x, \varepsilon) \text { is analytic in }(x, \varepsilon) \text { for } 0<|x| \leqslant R_{0 l}+f(\theta, \varepsilon), \quad|\varepsilon| \leqslant \varepsilon_{0}
\end{aligned}
$$

and these functions can actually be extended analytically into some $\delta_{0}$-neighbourhood of the free boundary where $\delta_{0}$ is a positive number independent of $\varepsilon$; here we use the uniform estimates on the derivatives in the $L^{\infty}$-norm (which follow by (8.1) and the Sobolev embedding).

It is now easy to extend both $\mu^{-}$and $u^{-}$as analytic functions in $(x, \varepsilon)$ also in a neighbourhood of $x=0$, as in [5]. Indeed, since $\mu^{-}$is bounded in $0<|x| \leqslant 1 / 2$, we can represent it in the form

$$
\mu^{-}(x, \varepsilon)=\int_{|x|=\delta} \frac{\partial G}{\partial n}(x-y) \mu^{-}(y, \varepsilon) \mathrm{d} S_{y}-\int_{|x|=\delta} G(x-y) \frac{\partial}{\partial n} \mu^{-}(y, \varepsilon) \mathrm{d} S_{y}
$$

for $0<|x| \leqslant \delta(\delta<1 / 2)$ where $G$ is a fundamental solution of $\Delta-1$. The right-hand side provides the analytic extension of $\mu^{-}(x, \varepsilon)$ to $|x| \leqslant \delta,|\varepsilon| \leqslant \varepsilon_{0}$. Next, since $\mu^{-}(x, \varepsilon)+u(x, \varepsilon)$ is harmonic and bounded in $0<|x| \leqslant 1 / 2$, we can similarly extend it to $|x| \leqslant \delta,|\varepsilon| \leqslant \varepsilon_{0}$, and thus conclude that the function $u(x, \varepsilon)$ also has analytic extension to $|x| \leqslant \delta,|\varepsilon| \leqslant \varepsilon_{0}$.

We have thus completed the proof of the following theorem, which is the main result of this paper.

THEOREM 8.1 For any integer $l \geqslant 2$ there exists a family of solutions of (1.1)-(1.6) with free boundary $r=R_{0 l}+f(\theta, \varepsilon)$ where

$$
f(\theta, \varepsilon)=\varepsilon \cos l \theta+\mathrm{O}\left(\varepsilon^{2}\right) .
$$


The solution $\mu, u, r=R_{0 l}+f(\theta, \varepsilon), \beta$ has the form (1.18), (1.19), (1.7), (1.8) and the series converge and define analytic function in $(x, \varepsilon)$ for $\mu, u$ and in $(\theta, \varepsilon)$ for $f$; more precisely

$$
\begin{aligned}
& \mu^{+}(x, \varepsilon) \text { is analytic in }|x| \geqslant R_{0 l}-\delta_{0}, \quad|\varepsilon| \leqslant \varepsilon_{0}, \\
& \mu^{-}(x, \varepsilon) \text { and } u(x, \varepsilon) \text { are analytic in }|x| \leqslant R_{0 l}+\delta_{0}, \quad|\varepsilon| \leqslant \varepsilon_{0},
\end{aligned}
$$

for some $\varepsilon_{0}>0, \delta_{0}>0$; furthermore, the solution is even in $\theta$ and satisfies the conditions (1.9)(1.11). Finally, $R_{0 l}$ and the solution with all the above propertied are unique.

\section{Acknowledgement}

AF is partially supported by National Science Foundation Grant DMS \#9703842. BM and JJLV are grateful for a partial support from the Institute for Mathematics and its Application during their visit there. JJLV is partially supported by DGICYT grant PB96-0614.

\section{REFERENCES}

1. AdAms, R. A. Sobolev Spaces. Academic, New York (1975).

2. Friedman, A. On the regularity of solutions on nonlinear elliptic and parabolic equations. J. Math. Mech. 7, (1958) 43-60.

3. Friedman, A. \& Hu, B. A Stefan problem for a protocell model. SIAM J. Math. Anal. 30, (1998) 912-926.

4. Friedman, A., Hu, B., \& Velazquez, J. J. L. On the zeros of quotients of Bessel functions. Chinese Annals of Mathematics 21, (2000) 285-296.

5. Friedman, A. \& Reitich, F. Symmetry-breaking bifurcation of analytic solutions to free boundary problems: an application to a model of tumor growth. Trans. Amer. Math. Soc., to appear.

6. Sattinger, D. H. Group Theoretic Methods in Bifurcation Theory, (Lecture Notes in Mathematics). Springer, Berlin (1979).

7. Smoller, J. Shock Waves and Reaction Diffusion Equations. Springer, New York (1983).

8. Schwegler, H., TARUmi, K., \& Gerstmann, B. Physico-chemical model of protocell. J. Math. Biol. 22, (1985) 335-348.

9. TARUMi, K. \& SCHWEgLER, H. A nonlinear treatment of the protocell by boundary layer approximation. Bull. Math. Biol. 49, (1987) 307-320.

10. Watson, G. N. A Treatise on the Theory of Functions, 2nd edn. Cambridge University Press, Cambridge (1944).

\section{Appendix A. Some facts about Bessel functions}

We collect several facts about the Bessel functions $I_{m}(x)$ for $m \geqslant 0, x \geqslant 0$. We recall that $I_{m}(x)$ satisfies the differential equation

$$
I_{m}^{\prime \prime}(x)+\frac{1}{r} I_{m}^{\prime}(x)-\left(1+\frac{m^{2}}{x^{2}}\right) I_{m}(x)=0
$$

and is given by

$$
I_{m}(x)=\sum_{k=0}^{\infty} \frac{(x / 2)^{m+2 k}}{k ! \Gamma(m+k+1)} .
$$


Furthermore,

$$
\begin{aligned}
& I_{m}^{\prime}(x)+\frac{m}{x} I_{m}(x)=I_{m-1}(x), \quad m \geqslant 1, \\
& I_{m}^{\prime}(x)-\frac{m}{x} I_{m}(x)=I_{m+1}(x),
\end{aligned}
$$

so that

$$
I_{m-1}(x)-I_{m+1}(x)=\frac{2 m}{x} I_{m}(x), \quad m \geqslant 1 .
$$

We also recall [10: p. 225] the relation

$$
I_{m}(x)=\sqrt{\frac{1}{2 m \pi}}\left(\frac{e x}{2 m}\right)^{m}\left(1+\mathrm{O}\left(\frac{1}{m}\right)\right) \quad m \rightarrow \infty
$$

uniformly in $x$ in any bounded set.

We finally state three recent results.

TheOREM A.1 ([4]) For any $m \geqslant 2$,

$$
\frac{\mathrm{d}}{\mathrm{d} x}\left(\frac{I_{0}(x)}{I_{1}(x)} \frac{I_{m}(x)}{I_{m-1}(x)}\right)>0 \quad \text { for } \quad 0<x<\infty .
$$

THEOREM A.2 ([4]) For any $m \geqslant 2$ there exists a unique positive solution $x=x_{m}$ of the equation

$$
\frac{I_{0}(x)}{I_{1}(x)} \frac{I_{m}(x)}{I_{m-1}(x)}=\frac{m+1}{2 m},
$$

and

$$
x_{l}<x_{m} \quad \text { if } \quad 2 \leqslant l \leqslant m .
$$

THEOREM A.3 ([5]) The following identity holds for any $m \geqslant 2$ :

$\frac{1}{x^{2}} \int_{0}^{x}\left[m^{2} I_{1}(s) I_{m}(s)-2 s I_{0}(s) I_{m}(s)\right] \mathrm{d} s-\frac{m-2}{x} I_{1}(x) I_{m}(x)+\left[I_{2}(x) I_{m}(x)-I_{1}(x) I_{m+1}(x)\right] \equiv 0$.

\section{Appendix B. Some lemmas on analyticity}

In this section we establish norm estimates on the derivatives of composite functions of the same type as appeared in [1] and [5: Section 9].

Throughout this section we assume that the norm $\|\cdot\|$ satisfies the property

$$
\|f g\| \leqslant\|f\|\|g\| \text {. }
$$

LEMMA B. 1 Suppose

$$
\left\|\frac{1}{k !} \partial_{\theta}^{k} w_{m}^{(i)}(\theta)\right\| \leqslant \frac{B^{k}}{k^{2}} \frac{H_{0} H^{m-1}}{m^{2}} \quad\left(k^{2}=1 \text { if } k=0\right)
$$


for all $m \geqslant 1,0 \leqslant k \leqslant K, 1 \leqslant i \leqslant q$ and set

$$
\prod_{i=1}^{q}\left(\sum_{m=1}^{\infty} w_{m}^{(i)}(\theta) \varepsilon^{m}\right)=\sum_{m=q}^{\infty} W_{m}^{q}(\theta) \varepsilon^{m} \quad(q=1,2,3, \ldots) .
$$

Then

$$
\left\|\frac{1}{k !} \partial_{\theta}^{k} W_{m}^{q}(\theta)\right\| \leqslant \frac{B^{k}}{k^{2}} \frac{H_{0} H^{m-1}}{m^{2}}\left(\frac{48 H_{0}}{H}\right)^{q-1} \quad \text { for } \quad m \geqslant q, 0 \leqslant k \leqslant K .
$$

Proof. First, we have

$$
\begin{aligned}
& \sum_{k=1}^{m-1} \frac{1}{k^{2}(m-k)^{2}}=\sum_{k=1}^{m-1}\left(\frac{1}{m^{2} k^{2}}+\frac{2}{m^{3} k}+\frac{1}{m^{2}(m-k)^{2}}+\frac{2}{m^{3}(m-k)^{3}}\right) \\
& \leqslant 2 \sum_{k=1}^{m-1}\left(\frac{1}{m^{2} k^{2}}+\frac{2}{m^{3} k}\right)<\frac{2}{m^{2}}\left\{\sum_{k=1}^{\infty} \frac{1}{k^{2}}+\frac{2}{m^{2}}\left(1+\int_{1}^{m-1} \frac{\mathrm{d} x}{x}\right)\right\} \\
& \quad=\frac{2}{m^{2}}\left\{\frac{\pi^{2}}{6}+\frac{2}{m}[1+\ln (m-1]\} \leqslant \frac{2}{m^{2}}\left\{\frac{\pi^{2}}{6}+\frac{2}{3}(1+\ln 2)\right\}<\frac{6}{m^{2}} .\right.
\end{aligned}
$$

The estimate (B.3) is valid for $q=1$, by assumption. We proceed by induction from $q-1$ to $q$. By (B.4), we also have (recall the convention $k^{2}=1$ if $k=0$ )

$$
\sum_{k=0}^{m} \frac{1}{k^{2}(m-k)^{2}}=\frac{2}{m^{2}}+\sum_{k=1}^{m-1} \frac{1}{k^{2}(m-k)^{2}} \leqslant \frac{8}{m^{2}}
$$

Note that

$$
W_{m}^{q}(\theta)=\sum_{j=1}^{m-q+1} W_{m-j}^{q-1}(\theta) w_{j}^{(q)}(\theta)
$$

and

$$
\frac{1}{k !} \partial_{\theta}^{k} W_{m}^{q}(\theta)=\sum_{j=1}^{m-q+1} \sum_{l=0}^{k} \frac{1}{l !} \partial_{\theta}^{l} W_{m-j}^{q-1} \frac{1}{(k-l) !} \partial_{\theta}^{k-l} w_{j}^{(q)}
$$

Therefore

$$
\begin{aligned}
\left\|\frac{1}{k !} \partial_{\theta}^{k} W_{m}^{q}(\theta)\right\| & \leqslant \sum_{j=1}^{m-q+1} \sum_{l=0}^{k} \frac{B^{l}}{l^{2}} \frac{H_{0} H^{m-j-1}}{(m-j)^{2}}\left(\frac{48 H_{0}}{H}\right)^{q-2} \frac{B^{k-l}}{(k-l)^{2}} \frac{H_{0} H^{j-1}}{j^{2}} \\
& \leqslant B^{k} H_{0} H^{m-1}\left(\frac{48 H_{0}}{H}\right)^{q-1} \frac{1}{48} \sum_{j=1}^{m-q+1} \frac{1}{(m-j)^{2}} \frac{1}{j^{2}} \sum_{l=0}^{k} \frac{1}{l^{2}} \frac{1}{(k-l)^{2}} \\
& \leqslant \frac{B^{k}}{k^{2}} \frac{H_{0} H^{m-1}}{m^{2}}\left(\frac{48 H_{0}}{H}\right)^{q-1} \quad \text { (by (B.4) and (B.5)). }
\end{aligned}
$$


Taking $w_{m}^{(1)}(\theta)=w_{m}^{(2)}(\theta)=\cdots=w_{m}^{(q)}(\theta)=w_{m}(\theta)$ in Lemma B.1, we get Lemma B.2 Suppose

$$
\left\|\frac{1}{k !} \partial_{\theta}^{k} w_{m}(\theta)\right\| \leqslant \frac{B^{k}}{k^{2}} \frac{H_{0} H^{m-1}}{m^{2}} \quad\left(k^{2}=1 \text { if } k=0\right)
$$

for all $m \geqslant 1,0 \leqslant k \leqslant K$ and set

$$
\left(\sum_{m=1}^{\infty} w_{m}(\theta) \varepsilon^{m}\right)^{q}=\sum_{m=q}^{\infty} W_{m}^{q}(\theta) \varepsilon^{m} \quad(q=1,2,3, \ldots) .
$$

Then

$$
\left\|\frac{1}{k !} \partial_{\theta}^{k} W_{m}^{q}(\theta)\right\| \leqslant \frac{B^{k}}{k^{2}} \frac{H_{0} H^{m-1}}{m^{2}}\left(\frac{48 H_{0}}{H}\right)^{q-1} \quad \text { for } \quad m \geqslant q, \quad 0 \leqslant k \leqslant K .
$$

Lemma B.3 Consider the formal power series

$$
\begin{aligned}
& f(w, \theta)=\sum_{m=1}^{\infty} f_{m}(\theta) w^{m}, \\
& w(\theta, \varepsilon)=\sum_{m=1}^{\infty} w_{m}(\theta) \varepsilon^{m}
\end{aligned}
$$

and set

$$
F(\theta, \varepsilon)=f[w(\theta, \varepsilon), \theta]=\sum_{m=1}^{\infty} F_{m}(\theta) \varepsilon^{m} .
$$

Suppose that

$$
\left\|\frac{1}{k !} \partial_{\theta}^{k} f_{m}\right\| \leqslant A_{0} A^{m-1} \frac{B^{k}}{k^{2}}, \quad\left\|\frac{1}{k !} \partial_{\theta}^{k} w_{m}\right\| \leqslant \frac{B^{k}}{k^{2}} \frac{H_{0} H^{m-1}}{m^{2}}
$$

hold for all $m \geqslant 1$ and all $0 \leqslant k \leqslant K$ and $H / A \geqslant 96 H_{0}$. Then

$$
\left\|\frac{1}{k !} \partial_{\theta}^{k} F_{m}\right\| \leqslant 16 A_{0} \frac{B^{k}}{k^{2}} \frac{H_{0} H^{m-1}}{m^{2}} \quad \text { for } \quad m \geqslant 1 .
$$

Proof. Using the notation from previous lemmas, we have

$$
F_{m}=\sum_{q=1}^{m} f_{q} W_{m}^{q}
$$

Thus

$$
\frac{1}{k !} \partial_{\theta}^{k} F_{m}=\sum_{q=1}^{m} \sum_{l=0}^{k} \frac{1}{l !} \partial_{\theta}^{l} f_{q} \frac{1}{(k-l) !} \partial_{\theta}^{k-l} W_{m}^{q}
$$


We now estimate

$$
\begin{aligned}
\left\|\frac{1}{k !} \partial_{\theta}^{k} F_{m}\right\| & \leqslant \sum_{q=1}^{m} \sum_{l=0}^{k} A_{0} A^{q-1} \frac{B^{l}}{l^{2}} \cdot \frac{B^{k-l}}{(k-l)^{2}} \frac{H_{0} H^{m-1}}{m^{2}}\left(\frac{48 H_{0}}{H}\right)^{q-1} \\
& \leqslant \frac{8 A_{0} B^{k}}{k^{2}} \cdot \frac{H_{0} H^{m-1}}{m^{2}} \sum_{q=1}^{\infty} \frac{1}{2^{q-1}} \quad(\text { by (B.5)) } \\
& =16 \frac{A_{0} B^{k}}{k^{2}} \cdot \frac{H_{0} H^{m-1}}{m^{2}} .
\end{aligned}
$$

The above lemma extends to double power series.

LEMMA B.4 Consider the formal power series

$$
\begin{aligned}
& f(x, y, \theta)=\sum_{1 \leqslant i+j<\infty} f_{i j}(\theta) x^{i} y^{j} \\
& w_{1}(\theta, \varepsilon)=\sum_{m=1}^{\infty} w_{m}^{(1)}(\theta) \varepsilon^{m} \\
& w_{2}(\theta, \varepsilon)=\sum_{m=1}^{\infty} w_{m}^{(2)}(\theta) \varepsilon^{m}
\end{aligned}
$$

and set

$$
F(\theta, \varepsilon)=f\left[w_{1}(\theta, \varepsilon), w_{2}(\theta, \varepsilon), \theta\right]=\sum_{m=1}^{\infty} F_{m}(\theta) \varepsilon^{m}
$$

Suppose that

$$
\left\|\frac{1}{k !} \partial_{\theta}^{k} f_{i j}\right\| \leqslant A_{0} A^{i+j-1} \frac{B^{k}}{k^{2}}, \quad\left\|\frac{1}{k !} \partial_{\theta}^{k} w_{m}^{(i)}\right\| \leqslant \frac{B^{k}}{k^{2}} \frac{H_{0} H^{m-1}}{m^{2}}, \quad i=1,2 .
$$

hold for all $m \geqslant 1, i+j \geqslant 1$ and all $k \geqslant 0$ and $H / A \geqslant 96 H_{0}$. Then

$$
\left\|\frac{1}{k !} \partial_{\theta}^{k} F_{m}\right\| \leqslant 32 \cdot A_{0} \frac{B^{k}}{k^{2}} \frac{H_{0} H^{m-1}}{m^{2}} .
$$

If, furthermore,

$$
f_{10}(\theta) \equiv f_{01}(\theta) \equiv 0,
$$

i.e. the series for $f$ starts with second-order terms, then

$$
\left\|\frac{1}{k !} \partial_{\theta}^{k} F_{m}\right\| \leqslant 32 \cdot 48 A_{0} \frac{B^{k}}{k^{2}} \frac{H_{0}^{2} H^{m-2}}{m^{2}} .
$$

Similarly, if the series for $f$ starts with mth order terms, then there is an extra $\left(48 H_{0} / H\right)^{m-1}$ factor in the resulting estimates (right-hand side of (B.11)). 
Proof. We write

$$
\left(\sum_{m=1}^{\infty} w_{m}^{(1)}(\theta) \varepsilon^{m}\right)^{i}\left(\sum_{m=1}^{\infty} w_{m}^{(2)}(\theta) \varepsilon^{m}\right)^{j}=\sum_{m=i+j}^{\infty} F_{m}^{(i, j)}(\theta) \varepsilon^{m}
$$

Then by Lemma B.1,

$$
\left\|\frac{1}{k !} \partial_{\theta}^{k} F_{m}^{(i, j)}\right\| \leqslant \frac{B^{k}}{k^{2}} \frac{H_{0} H^{m-1}}{m^{2}}\left(\frac{48 H_{0}}{H}\right)^{i+j-1} .
$$

Since

$$
F_{m}(\theta)=\sum_{1 \leqslant i+j \leqslant m} f_{i j}(\theta) F_{m}^{(i, j)}(\theta)
$$

we then have

$$
\begin{aligned}
\left\|\frac{1}{k !} \partial_{\theta}^{k} F_{m}(\theta)\right\| & \leqslant \sum_{1 \leqslant i+j \leqslant m} \sum_{l=0}^{k}\left\|\frac{1}{l !} \partial_{\theta}^{l} f_{i j}(\theta)\right\|\left\|\frac{1}{(k-l) !} \partial_{\theta}^{k-l} F_{m}^{(i, j)}\right\| \\
& \leqslant \sum_{1 \leqslant i+j \leqslant m} \sum_{l=0}^{k} A_{0} A^{i+j-1} \frac{B^{l}}{l^{2}} \frac{B^{k-l}}{(k-l)^{2}} \frac{H_{0} H^{m-1}}{m^{2}}\left(\frac{48 H_{0}}{H}\right)^{i+j-1} \\
& \leqslant 8 A_{0} \frac{B^{k}}{k^{2}} \frac{H_{0} H^{m-1}}{m^{2}} \sum_{1 \leqslant i+j \leqslant m}\left(\frac{1}{2}\right)^{i+j-1} \quad \text { (by (B.5)) } \\
& =32 \cdot A_{0} \frac{B^{k}}{k^{2}} \frac{H_{0} H^{m-1}}{m^{2}} .
\end{aligned}
$$

In the case $f_{10}(\theta) \equiv f_{01}(\theta) \equiv 0, i+j$ is at least 2 for the nonvanishing terms and there is an extra factor $48 H_{0} / H$ factor. Similarly if $i+j$ starts with $m$, then we get the extra factor $\left(48 H_{0} / H\right)^{m-1}$.

In the next lemma, we use two norms, $\|\cdot\|_{X_{1}}$ for functions $g(\theta)$ and $\|\cdot\|_{X_{2}}$ for functions $f(r, \theta)$. LEMMA B.5 Suppose that the norms $\|\cdot\|_{X_{1}}$ and $\|\cdot\|_{X_{2}}$ satisfy the following algebra property:

$$
\|g(\theta) f(r, \theta)\|_{X_{2}} \leqslant C^{*}\|g\|_{X_{1}}\|f\|_{X_{2}} .
$$

Assume that

$$
\left\|\partial_{\theta}^{k} w_{m}(\theta)\right\|_{X_{1}} \leqslant k ! \frac{B^{k}}{k^{2}} \frac{H_{0} H^{m-1}}{m^{2}} \quad\left(k^{2}=1 \quad \text { if } \quad k=0\right)
$$

for all $m \geqslant 1,0 \leqslant k \leqslant K$ and fixed $i$, and

$$
\left\|\partial_{r}^{i} \partial_{\theta}^{k} u_{m}(r, \theta)\right\|_{X_{2}} \leqslant(k+i) ! \frac{B^{k}}{k^{2}} \frac{H_{0} H^{m-1}}{m^{2}} .
$$

Set

$$
\left(\sum_{m=1}^{\infty} w_{m}(\theta) \varepsilon^{m}\right)\left(\sum_{m=1}^{\infty} u_{m}(r, \theta) \varepsilon^{m}\right)=\sum_{m=2}^{\infty} U_{m}(r, \theta) \varepsilon^{m}
$$


Then

$$
\left\|\partial_{r}^{i} \partial_{\theta}^{k} U_{m}(r, \theta)\right\|_{X_{2}} \leqslant C^{*}(k+i) ! \frac{B^{k}}{k^{2}} \frac{H_{0} H^{m-1}}{m^{2}}\left(\frac{48 H_{0}}{H}\right) \quad \text { for } \quad m \geqslant 2,0 \leqslant k \leqslant K . \quad \text { (B.16) }
$$

Proof. The proof is almost the same as that for Lemma B.1. Note that

$$
U_{m}(r, \theta)=\sum_{j=1}^{m-1} w_{m-j}(\theta) u_{j}(r, \theta)
$$

and

$$
\partial_{r}^{i} \partial_{\theta}^{k} U_{m}(r, \theta)=\sum_{j=1}^{m-1} \sum_{l=0}^{k}\left(\begin{array}{l}
k \\
l
\end{array}\right) \partial_{\theta}^{l} w_{m-j}(\theta) \partial_{r}^{i} \partial_{\theta}^{k-l} u_{j}(r, \theta)
$$

Therefore

$$
\begin{aligned}
& \left\|\frac{1}{(k+i) !} \partial_{r}^{i} \partial_{\theta}^{k} U_{m}(r, \theta)\right\|_{X_{2}} \\
& \leqslant C^{*} \sum_{j=1}^{m-1} \sum_{l=0}^{k} \frac{\left(\begin{array}{c}
k \\
l
\end{array}\right)}{\left(\begin{array}{c}
k+i \\
l
\end{array}\right)} \frac{B^{l}}{l^{2}} \frac{H_{0} H^{m-j-1}}{(m-j)^{2}} \frac{B^{k-l}}{(k-l)^{2}} \frac{H_{0} H^{j-1}}{j^{2}} \\
& \leqslant C^{*} B^{k} H_{0} H^{m-1}\left(\frac{48 H_{0}}{H}\right) \frac{1}{48} \sum_{j=1}^{m-1} \frac{1}{(m-j)^{2}} \frac{1}{j^{2}} \sum_{l=0}^{k} \frac{1}{l^{2}} \frac{1}{(k-l)^{2}} \\
& \leqslant C^{*} \frac{B^{k}}{k^{2}} \frac{H_{0} H^{m-1}}{m^{2}}\left(\frac{48 H_{0}}{H}\right) .
\end{aligned}
$$

The proof is complete. 